\title{
Structure-property effects in the generation of transient aqueous benzoic acid anhydrides by carbodiimide fuels
}

\author{
Supporting Information
}

Lasith S. Kariyawasam, Julie C. Kron, Run Jiang, André J. Sommer, and C. Scott Hartley*

Department of Chemistry \& Biochemistry, Miami University, Oxford, OH 45056, USA

\section{Table of Contents}

IR spectroscopy monitoring $\ldots \ldots \ldots \ldots \ldots \ldots \ldots \ldots \ldots \ldots \ldots \ldots$

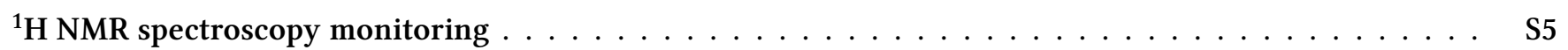

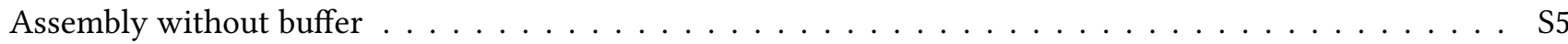

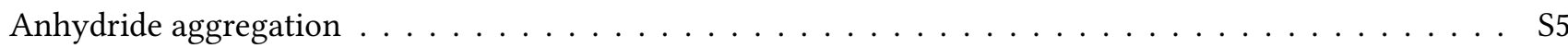

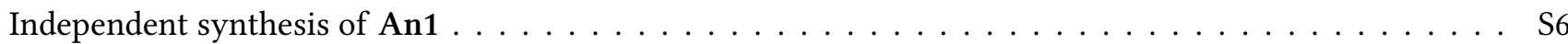

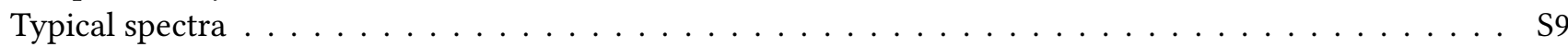

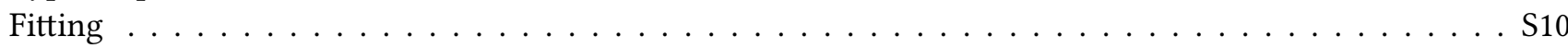

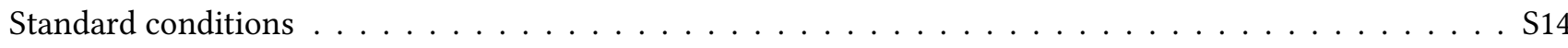

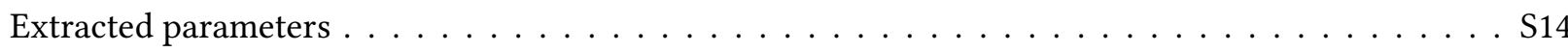

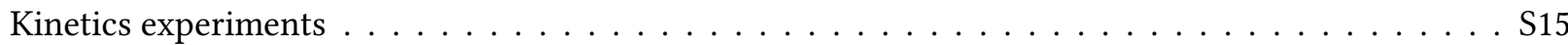

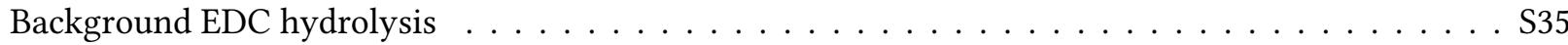

Variation of $\mathrm{pD}$ and buffer concentration $\ldots \ldots \ldots \ldots \ldots \ldots \ldots \ldots \ldots \ldots \ldots$

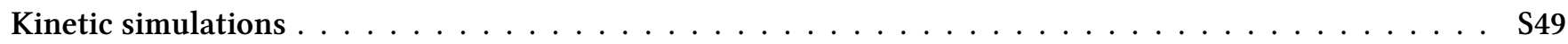

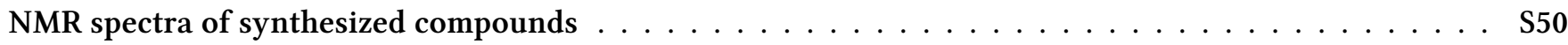

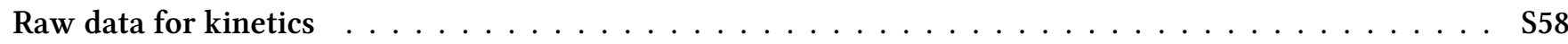

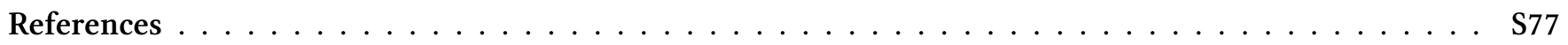

\section{List of Figures}

S1 IR spectroscopy monitoring of the treatment of Ac1 and Ac4 with EDC . . . . . . . . . . . S4

S2 IR spectroscopy monitoring of the treatment of Ac2 with EDC. . . . . . . . . . . . . S4

S3 IR spectroscopy monitoring of the treatment of Ac3 with EDC. . . . . . . . . . . . . . . S5

S4 Representative ${ }^{1} \mathrm{H}$ NMR spectrum for the treatment of Ac2 with EDC (1 equiv) in the absence of

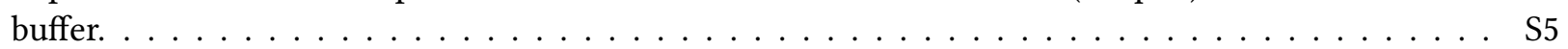

S5 Experimental chemical shift $(\delta)$ vs concentration for An2 and estimated fraction of free monomer. . S6

S6 Experimental chemical shift $(\delta)$ vs concentration for An4 and estimated fraction of free monomer. . S6

S7 $\quad{ }^{1} \mathrm{H}$ NMR spectrum $\left(500 \mathrm{MHz}, \mathrm{D}_{2} \mathrm{O}\right)$ of independently synthesized An1 . . . . . . . . . . . . S7

S8 $\quad{ }^{13} \mathrm{C}\left\{{ }^{1} \mathrm{H}\right\}$ NMR spectrum $\left(125 \mathrm{MHz}, \mathrm{D}_{2} \mathrm{O}\right)$ of independently synthesized An1 . . . . . . . . . . . . S8

S9 ${ }^{13} \mathrm{C}\left\{{ }^{1} \mathrm{H}\right\} \mathrm{NMR}$ spectrum $\left(125 \mathrm{MHz}, \mathrm{D}_{2} \mathrm{O}\right)$ of the Ac1/An1 system after treatment with EDC, showing signals matching those of independently prepared An1 . . . . . . . . . . . . S S9

S10 $\quad{ }^{1} \mathrm{H}$ NMR spectra of An1 formation from Ac1 acquired during a typical kinetic run. . . . . . . . . . S9

S11 ${ }^{1}$ H NMR spectra of An3 formation from Ac3 acquired during a typical kinetic run. . . . . . . . . S10

S12 $\quad{ }^{1}$ H NMR spectra of An4 formation from Ac4 acquired during a typical kinetic run. . . . . . . . . S10

S13 Kinetic run for Ac2, fitting the mechanism to this single dataset. . . . . . . . . . . . . . S13 
S14 $\quad{ }^{1}$ H NMR spectroscopy monitoring of the treatment of Ac1 with EDC.

S15 Heat map of the response of the regression error function to variation of $k_{-2}$ and $\alpha$ for the Ac1/An1

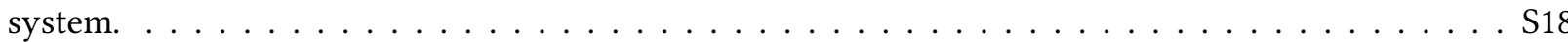

S16 Heat map of the response of the regression error function to variation of $k_{1}$ and $\alpha$ for the Ac1/An1

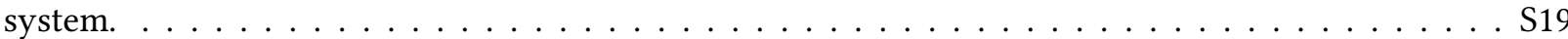

S17 Heat map of the response of the regression error function to variation of $k_{1}$ and $k_{-2}$ for the Ac1/An1

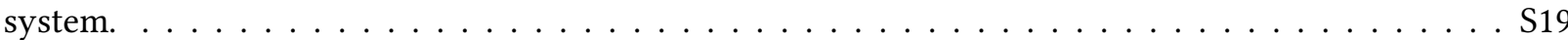

S18 ${ }^{1}$ H NMR spectroscopy monitoring of the treatment of Ac2 with EDC. . . . . . . . . . . . . . S20

S19 Heat map of the response of the regression error function to variation of $k_{-2}$ and $\alpha$ for the Ac2/An2

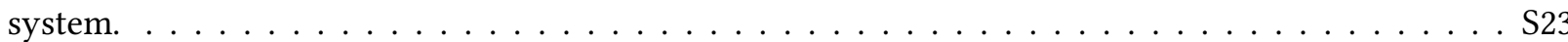

S20 Heat map of the response of the regression error function to variation of $k_{1}$ and $\alpha$ for the Ac2/An2

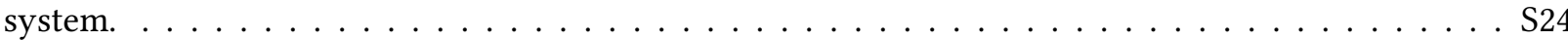

S21 Heat map of the response of the regression error function to variation of $k_{1}$ and $k_{-2}$ for the Ac2/An2

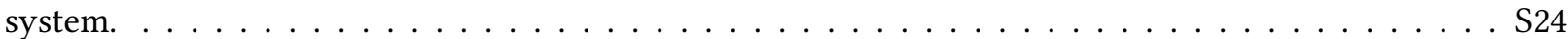

S22 ${ }^{1}$ H NMR spectroscopy monitoring of the treatment of Ac3 with EDC. . . . . . . . . . . . . . S25

S23 Heat map of the response of the regression error function to variation of $k_{-2}$ and $\alpha$ for the Ac3/An3

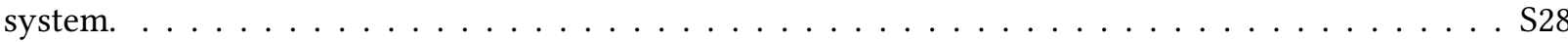

S24 Heat map of the response of the regression error function to variation of $k_{1}$ and $\alpha$ for the Ac3/An3

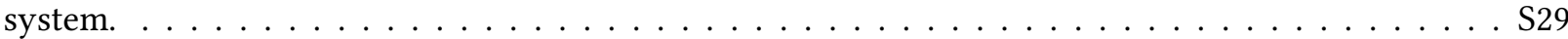

S25 Heat map of the response of the regression error function to variation of $k_{1}$ and $k_{-2}$ for the Ac3/An3

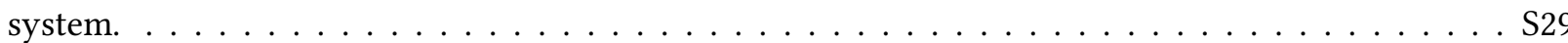

S26 ${ }^{1}$ H NMR spectroscopy monitoring of the treatment of Ac4 with EDC. . . . . . . . . . . . . . S30

S27 Heat map of the response of the regression error function to variation of $k_{-2}$ and $\alpha$ for the Ac4/An4

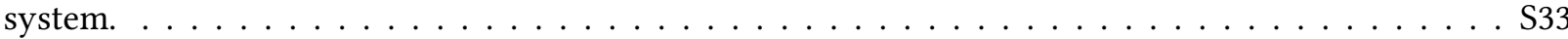

S28 Heat map of the response of the regression error function to variation of $k_{1}$ and $\alpha$ for the Ac4/An4

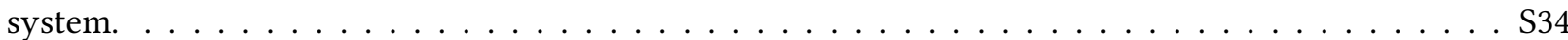

S29 Heat map of the response of the regression error function to variation of $k_{1}$ and $k_{-2}$ for the Ac4/An4

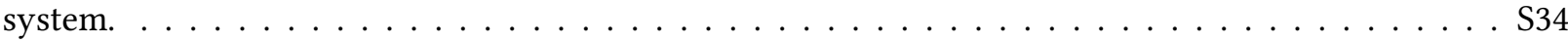

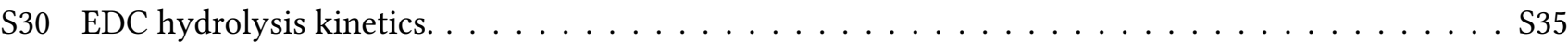

S31 ${ }^{1} \mathrm{H}$ NMR spectroscopy monitoring of the treatment of Ac1 with EDC at pD 5.0. . . . . . . . S36

S32 ${ }^{1} \mathrm{H}$ NMR spectroscopy monitoring of the treatment of Ac1 with EDC at pD 5.5. . . . . . . . . S37

S33 ${ }^{1} \mathrm{H}$ NMR spectroscopy monitoring of the treatment of Ac1 with EDC at 0.4 M pyridine- $d_{5}$ buffer. . . S38

S34 $\quad{ }^{1} \mathrm{H}$ NMR spectroscopy monitoring of the treatment of Ac1 with EDC at $0.6 \mathrm{M}$ pyridine- $d_{5}$ buffer. . . S39

S35 $\quad{ }^{1} \mathrm{H}$ NMR spectroscopy monitoring of the treatment of Ac2 with EDC at pD 5.0. . . . . . . . . S40

S36 $\quad{ }^{1} \mathrm{H}$ NMR spectroscopy monitoring of the treatment of Ac2 with EDC at $\mathrm{pD} 5.5 \ldots \ldots \ldots \ldots$. . . . S41

S37 ${ }^{1} \mathrm{H}$ NMR spectroscopy monitoring of the treatment of Ac2 with EDC at 0.4 M pyridine- $d_{5}$ buffer. . . S42

S38 ${ }^{1} \mathrm{H}$ NMR spectroscopy monitoring of the treatment of Ac2 with EDC at $0.6 \mathrm{M}$ pyridine- $d_{5}$ buffer. . . S43

S39 ${ }^{1}$ H NMR spectroscopy monitoring of the treatment of Ac3 with EDC at pD 5.0. . . . . . . . . . S44

S40 $\quad{ }^{1} \mathrm{H}$ NMR spectroscopy monitoring of the treatment of Ac3 with EDC at pD 5.5. . . . . . . . . S45

S41 ${ }^{1} \mathrm{H}$ NMR spectroscopy monitoring of the treatment of Ac3 with EDC at $0.4 \mathrm{M}$ pyridine- $d_{5}$ buffer. . . S46

S42 $\quad{ }^{1} \mathrm{H}$ NMR spectroscopy monitoring of the treatment of Ac3 with EDC at $0.6 \mathrm{M}$ pyridine- $d_{5}$ buffer. . . S47

S43 ${ }^{1} \mathrm{H}$ NMR spectroscopy monitoring of the treatment of Ac4 with EDC at $0.4 \mathrm{M}$ pyridine- $d_{5}$ buffer. . . S48

S44 ${ }^{1} \mathrm{H}$ NMR spectroscopy monitoring of the treatment of Ac4 with EDC at $0.6 \mathrm{M}$ pyridine- $d_{5}$ buffer. . . S49

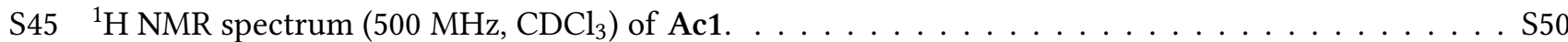

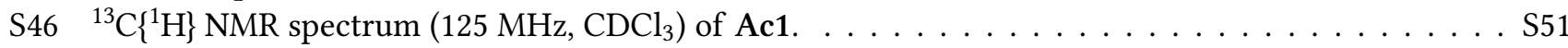

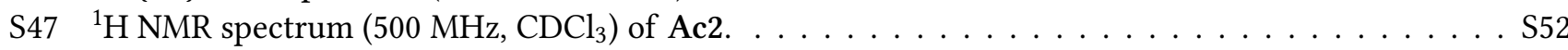

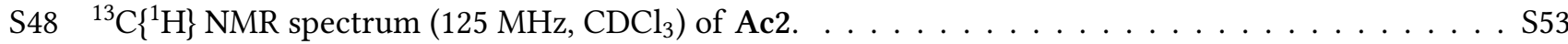

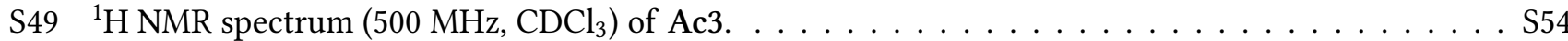

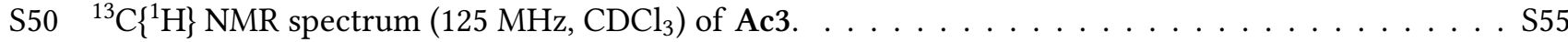

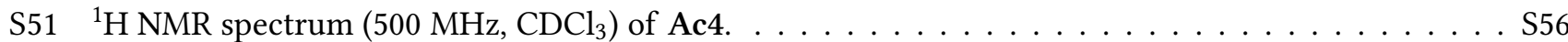




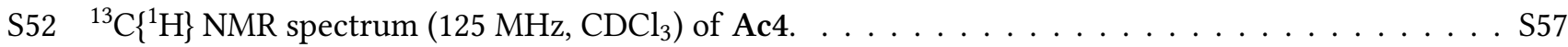

\section{List of Tables}

S1 Optimized parameters and confidence intervals obtained from the data in Figure S13. . . . . . . . S13

S2 Experimental kinetic parameters for anhydride formation and hydrolysis. . . . . . . . . . . . . S14

S3 Concentration vs time data for $45 \mathrm{mM} \mathrm{Ac1}$ with 0.50 equiv EDC. . . . . . . . . . . . . . . . S58

S4 Concentration vs time data for $45 \mathrm{mM} \mathrm{Ac1}$ with 0.75 equiv EDC. . . . . . . . . . . . . . . . . S58

S5 Concentration vs time data for $45 \mathrm{mM}$ Ac1 with 1.00 equiv EDC. . . . . . . . . . . . . . . . . . . S59

S6 Concentration vs time data for $12 \mathrm{mM} \mathrm{Ac1}$ with 0.75 equiv EDC. . . . . . . . . . . . . . . . . . S59

S7 Concentration vs time data for $25 \mathrm{mM} \mathrm{Ac1}$ with 0.75 equiv EDC. . . . . . . . . . . . . . . . . . S60

S8 Concentration vs time data for $30 \mathrm{mM} \mathrm{Ac1}$ with 1.00 equiv EDC. . . . . . . . . . . . . . . . S61

S9 Concentration vs time data for $30 \mathrm{mM}$ Ac1 with 0.75 equiv EDC. . . . . . . . . . . . . . . . . S62

S10 Concentration vs time data for $30 \mathrm{mM} \mathrm{Ac1}$ with 0.50 equiv EDC. . . . . . . . . . . . . . . S63

S11 Concentration vs time data for $45 \mathrm{mM} \mathrm{Ac2}$ with 0.50 equiv EDC. . . . . . . . . . . . . . . . . S64

S12 Concentration vs time data for $45 \mathrm{mM} \mathrm{Ac2}$ with 0.75 equiv EDC. . . . . . . . . . . . . . . . . S65

S13 Concentration vs time data for $45 \mathrm{mM} \mathrm{Ac2}$ with 1.00 equiv EDC. . . . . . . . . . . . . . . . S66

S14 Concentration vs time data for $25 \mathrm{mM} \mathrm{Ac2}$ with 0.75 equiv EDC. . . . . . . . . . . . . . . . . S66

S15 Concentration vs time data for $12 \mathrm{mM} \mathrm{Ac2}$ with 0.75 equiv EDC. . . . . . . . . . . . . . . . . S67

S16 Concentration vs time data for $30 \mathrm{mM} \mathrm{Ac2}$ with 1.00 equiv EDC. . . . . . . . . . . . . . . . S67

S17 Concentration vs time data for $30 \mathrm{mM} \mathrm{Ac2}$ with 0.75 equiv EDC. . . . . . . . . . . . . . . . . S68

S18 Concentration vs time data for $30 \mathrm{mM} \mathrm{Ac2}$ with 0.5 equiv EDC . . . . . . . . . . . . . . . . S68

S19 Concentration vs time data for $45 \mathrm{mM} \mathrm{Ac3}$ with 0.50 equiv EDC. . . . . . . . . . . . . . . . . . . S69

S20 Concentration vs time data for $45 \mathrm{mM} \mathrm{Ac3}$ with 0.75 equiv EDC. . . . . . . . . . . . . . . . . . S69

S21 Concentration vs time data for $45 \mathrm{mM} \mathrm{Ac3}$ with 1.00 equiv EDC. . . . . . . . . . . . . . . . . S70

S22 Concentration vs time data for $25 \mathrm{mM} \mathrm{Ac3}$ with 0.75 equiv EDC. . . . . . . . . . . . . . . . S70

S23 Concentration vs time data for $12 \mathrm{mM} \mathrm{Ac3}$ with 0.75 equiv EDC. . . . . . . . . . . . . . . . S71

S24 Concentration vs time data for $30 \mathrm{mM} \mathrm{Ac3}$ with 1.00 equiv EDC. . . . . . . . . . . . . . . . S71

S25 Concentration vs time data for $30 \mathrm{mM} \mathrm{Ac3}$ with 0.75 equiv EDC. . . . . . . . . . . . . . . . . . S72

S26 Concentration vs time data for $30 \mathrm{mM} \mathrm{Ac3}$ with 0.50 equiv EDC . . . . . . . . . . . . . . . S72

S27 Concentration vs time data for $45 \mathrm{mM} \mathrm{Ac4}$ with 0.50 equiv EDC. . . . . . . . . . . . . . . . S73

S28 Concentration vs time data for $45 \mathrm{mM} \mathrm{Ac4}$ with 0.75 equiv EDC. . . . . . . . . . . . . . . . S73

S29 Concentration vs time data for $45 \mathrm{mM} \mathrm{Ac4}$ with 1.00 equiv EDC. . . . . . . . . . . . . . . . S74

S30 Concentration vs time data for $30 \mathrm{mM}$ Ac4 with 1.00 equiv EDC. . . . . . . . . . . . . . . . . S74

S31 Concentration vs time data for $30 \mathrm{mM}$ Ac4 with 0.75 equiv EDC. . . . . . . . . . . . . . . . . . . S75

S32 Concentration vs time data for $30 \mathrm{mM}$ Ac4 with 0.50 equiv EDC. . . . . . . . . . . . . . . . S75

S33 Concentration vs time data for $75 \mathrm{mM}$ Ac4 with 0.50 equiv EDC. . . . . . . . . . . . . . . . . S76 


\section{IR spectroscopy monitoring}

IR spectroscopy experiments were carried out in unbuffered water as the presence of pyridine/pyridinium made it impossible to distinguish peaks of interest from background peaks. In all cases we observed characteristic signals for the symmetric stretching mode of the anhydrides near $1800 \mathrm{~cm}^{-1}$. On the time scale of the experiments $(1 \mathrm{~h})$, the hydrolysis of An4, An3, and An2 can be clearly observed although An1 is stable.

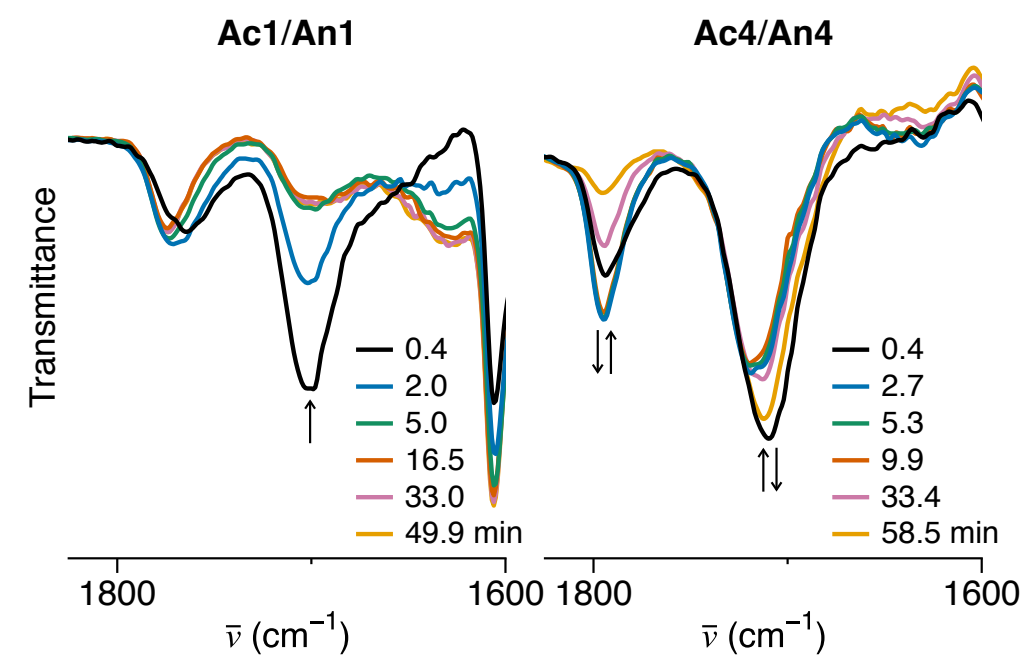

Figure S1. IR spectroscopy monitoring of the treatment of Ac1 and Ac4 with EDC.

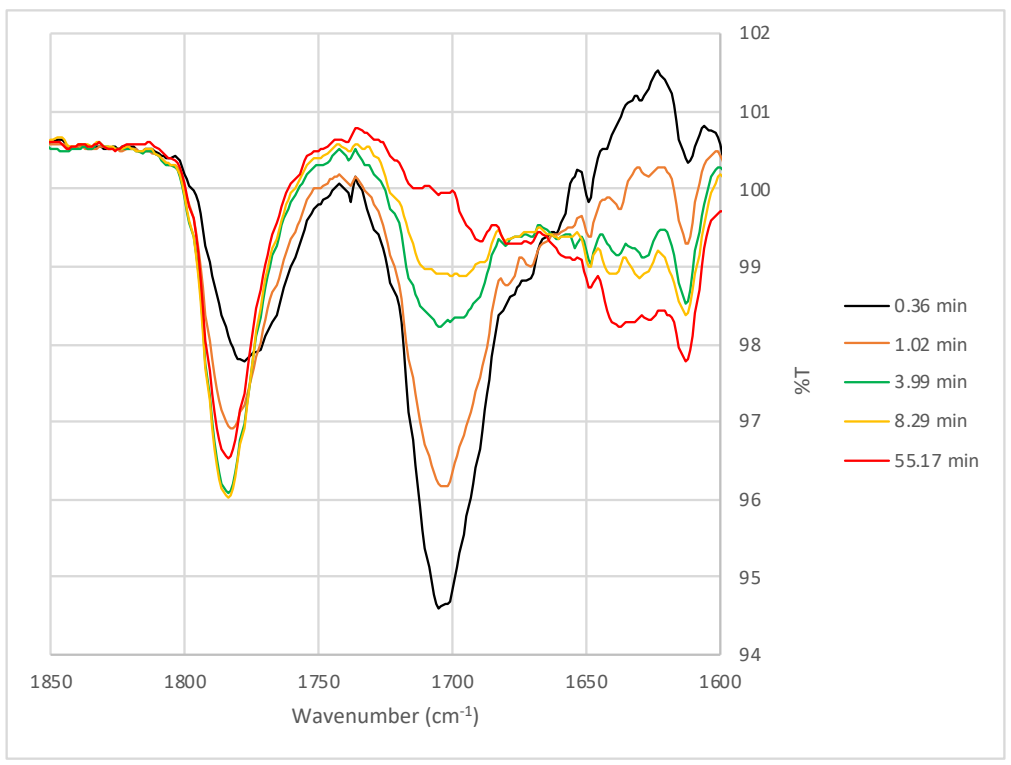

Figure S2. IR spectroscopy monitoring of the treatment of Ac2 with EDC. 


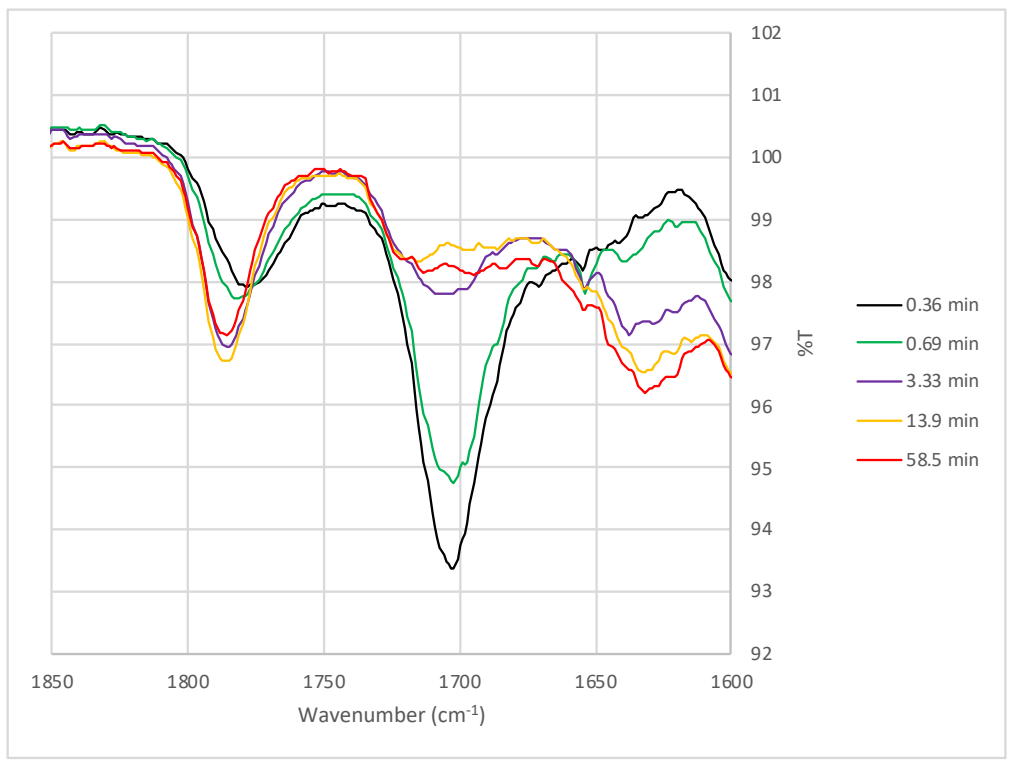

Figure S3. IR spectroscopy monitoring of the treatment of Ac3 with EDC.

\section{${ }^{1} \mathrm{H}$ NMR spectroscopy monitoring}

\section{Assembly without buffer}

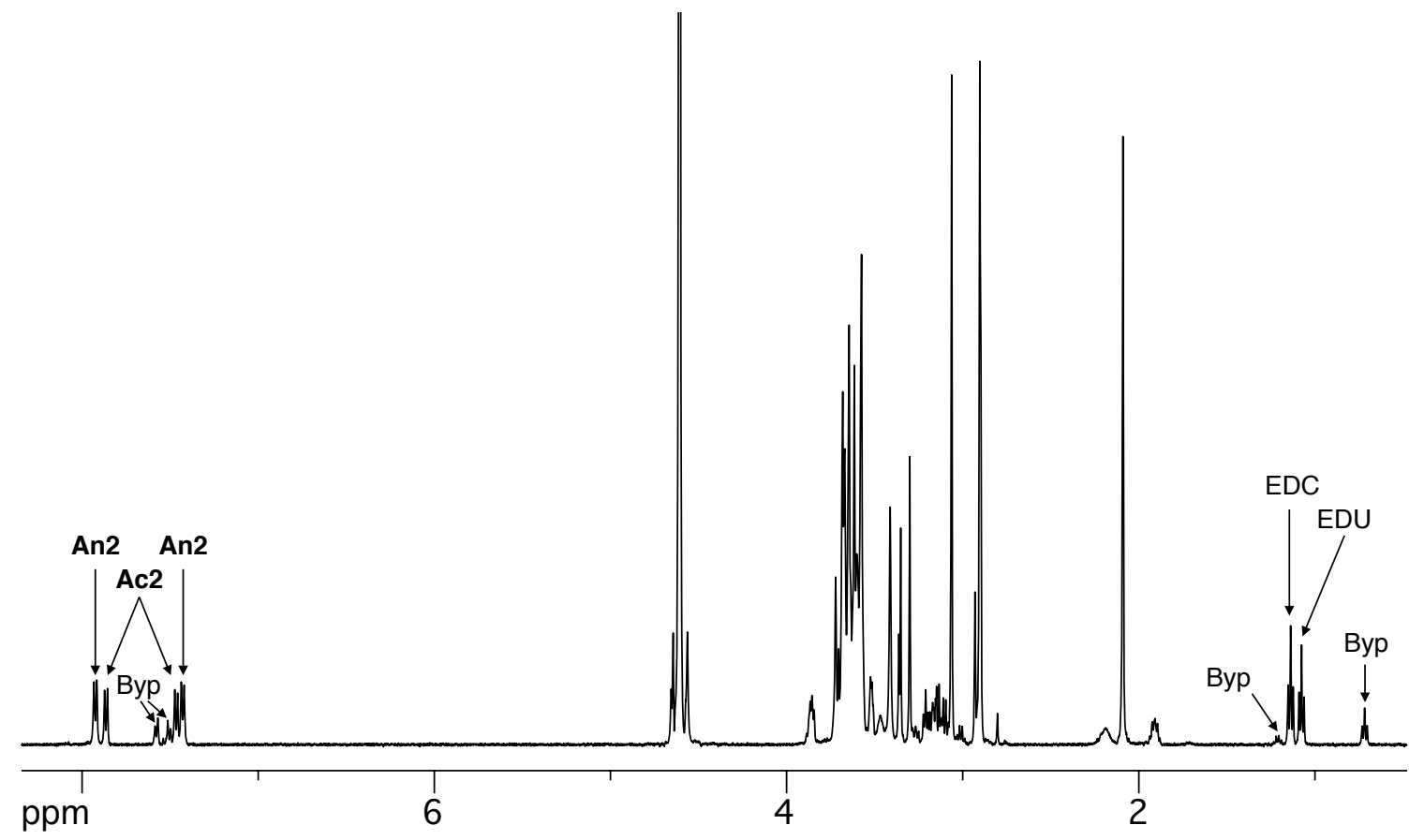

Figure S4. Representative ${ }^{1} \mathrm{H}$ NMR spectrum for the treatment of Ac2 with EDC (1 equiv) in the absence of buffer (initial $\mathrm{pD}$ adjusted to $4.5, I=1.0 \mathrm{M}, \mathrm{T}=298 \mathrm{~K}$ ). This spectrum was obtained $17 \mathrm{~min}$ after the addition of EDC and shows signals for An2 as well as two distinct byproducts ("Byp") that are likely $N$-acylureas.

\section{Anhydride aggregation}

In all four systems, the chemical shifts of the anhydrides were found to drift over the course of the experiments. The behavior of the systems was qualitatively similar. Compounds An2 and An4 yielded data from which associa- 
tion constants could be extracted with reasonable precision. The data was fit to the following equation: ${ }^{1}$

$$
\delta=\left(\delta_{d}-\delta_{m}\right)\left(1+\frac{1-\sqrt{8 K_{d} C+1}}{4 K_{d} C}\right)+\delta_{m}
$$

where $\delta$ is the observed chemical shift, $\delta_{d}$ is the chemical shift of the dimer, $\delta_{m}$ is the chemical shift of the monomer, $K_{d}$ is the dimerization constant, and $C$ is the concentration. The data alone cannot distinguish dimerization from an isodesmic indefinite polymerization; however, the relatively small changes in chemical shift suggest dimerization (0.8 ppm for An2, for which the best data was obtained). Fitting was carried out using a previously reported method implemented in Python $3 .^{2}$ The experimental data and the predicted fractions of free monomer are given in Figures S5 and S6.

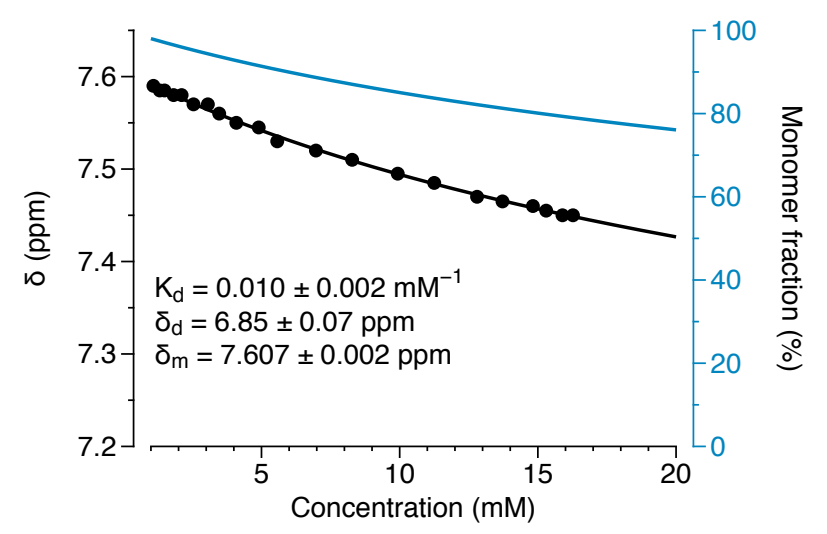

Figure S5. Experimental chemical shift $(\delta)$ vs concentration for An2 (black) and estimated fraction of free monomer (blue). The solid black line is a fit to eq 1 yielding the listed fit parameters. The data was extracted from an experiment on $\sim 50 \mathrm{mM}$ Ac2 and 1 equiv EDC.

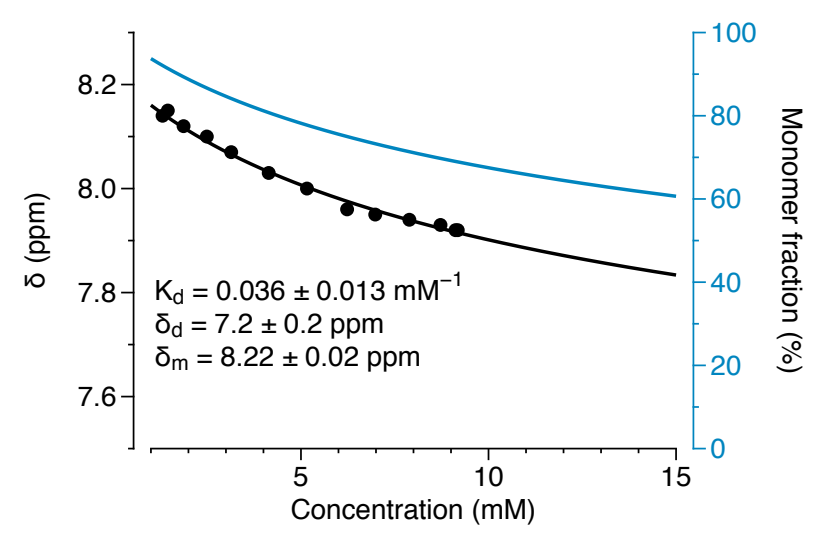

Figure S6. Experimental chemical shift $(\delta)$ vs concentration for An4 (black) and estimated fraction of free monomer (blue). The solid black line is a fit to eq 1 yielding the listed fit parameters. The data was extracted from an experiment on $\sim 50 \mathrm{mM}$ Ac4 and 1 equiv EDC.

\section{Independent synthesis of An1}

Ac1 (416 mg, $1.00 \mathrm{mmol}$ ) was placed in a glass vial equipped with a magnetic stir bar into which $\mathrm{CCl}_{4}(4 \mathrm{~mL})$ was added. $N, N^{\prime}$-Dicyclohexylcarbodiimide (DCC) $(104 \mathrm{mg}, 0.50 \mathrm{mmol})$ was added to the vial and the reaction mixture stirred at $\mathrm{rt}$ for $1 \mathrm{~h}$. It was then filtered, washing with $\mathrm{CCl}_{4}$ to remove the urea DCU. The filtrate was placed in the freezer overnight. The reaction mixture was concentrated under reduced pressure, resuspended in $\mathrm{CCl}_{4}$, and filtered again to remove DCU. The filtrate was again concentrated to yield crude An1 as a yellow oil (240 mg). The crude anhydride was not further purified, but was the principal component of the product as indicated by ${ }^{1} \mathrm{H}$ and ${ }^{13} \mathrm{C}\left\{{ }^{1} \mathrm{H}\right\}$ NMR spectroscopy (see Figures S7 and S8) and was detected by LRMS (ESI) (calcd for $\mathrm{C}_{40} \mathrm{H}_{62} \mathrm{O}_{17} \mathrm{Na}$ 
$\left(\left[\mathrm{M}^{+} \mathrm{Na}\right]^{+}\right)$837.4, found 837.6). Comparison the ${ }^{13} \mathrm{C}\left\{{ }^{1} \mathrm{H}\right\}$ NMR spectrum in $\mathrm{D}_{2} \mathrm{O}$ with that for the reaction of EDC with Ac1 demonstrates that the same transient species is generated in both cases.

\section{LSK-5-13-synthesized An1 in D2O}

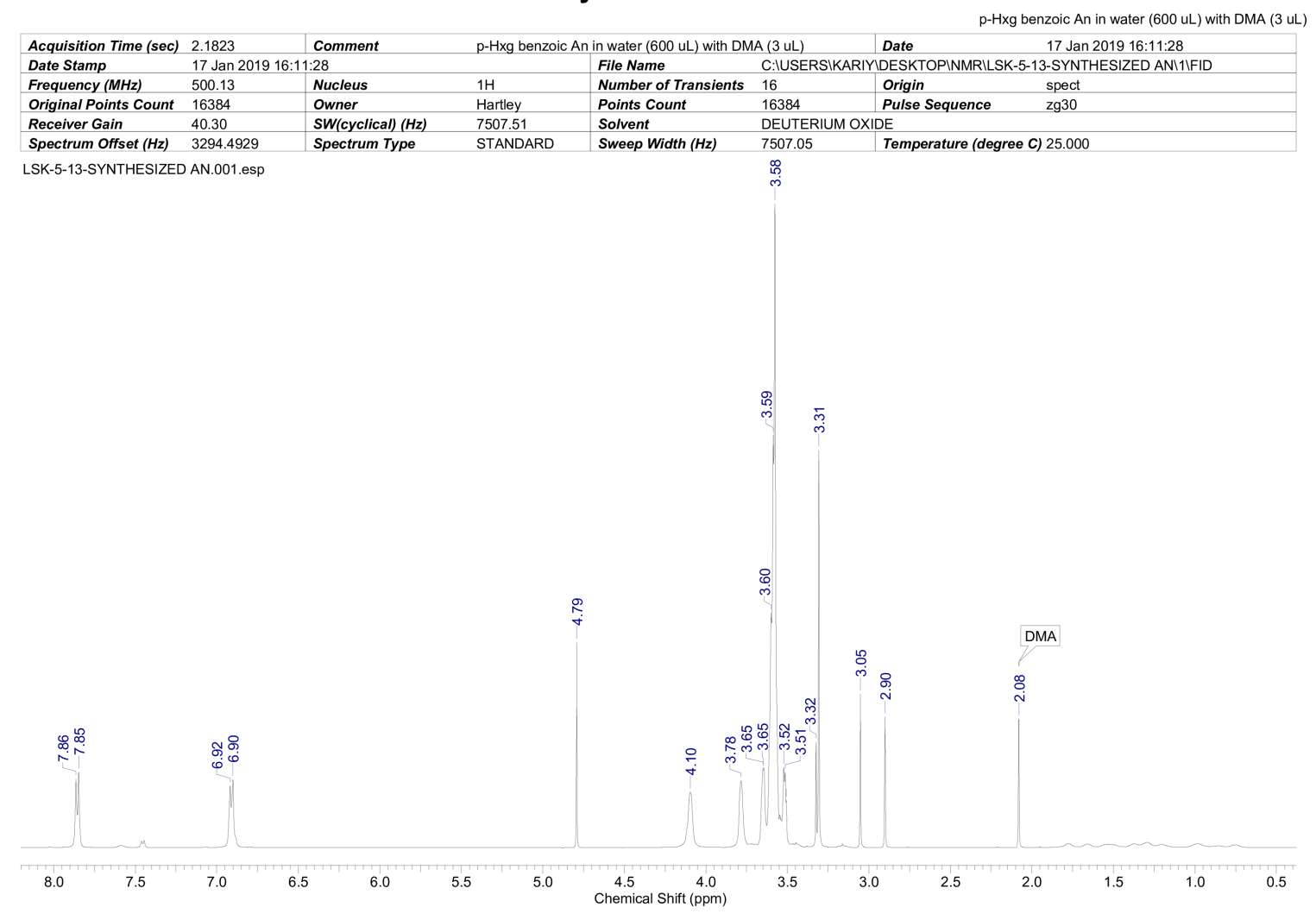

Figure S7. ${ }^{1} \mathrm{H}$ NMR spectrum $\left(500 \mathrm{MHz}, \mathrm{D}_{2} \mathrm{O}\right)$ of independently synthesized An1. 


\section{LSK-5-13-synthesized An1 in D2O}

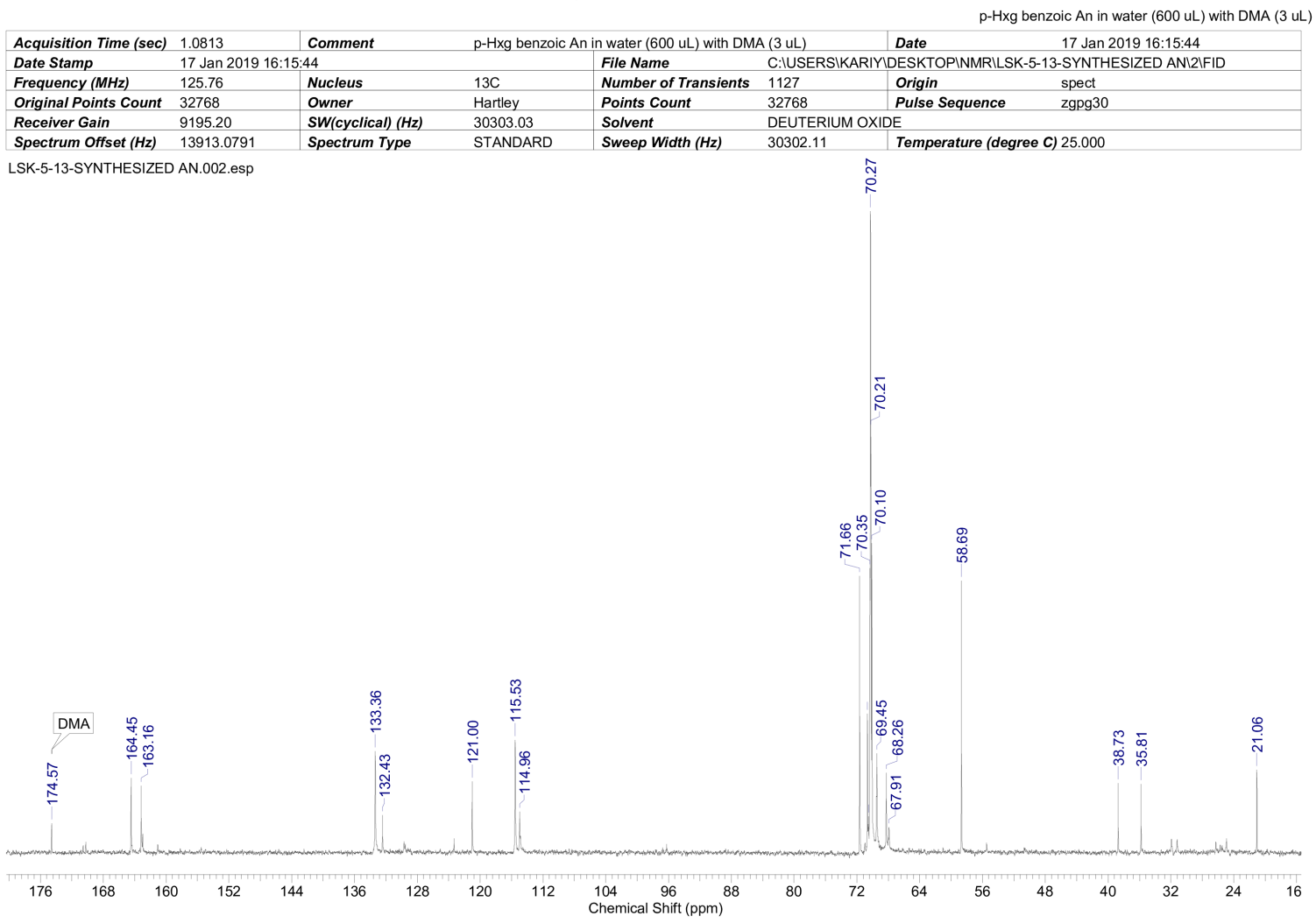

Figure S8. ${ }^{13} \mathrm{C}\left\{{ }^{1} \mathrm{H}\right\}$ NMR spectrum $\left(125 \mathrm{MHz}, \mathrm{D}_{2} \mathrm{O}\right)$ of independently synthesized $\mathrm{An} 1$. 


\section{LSK-5-16-Kinetic Run with Ac1}

\begin{tabular}{|c|c|c|c|c|c|c|c|}
\hline Acquisition Time (sec) & 1.0813 & Comment & \multicolumn{2}{|c|}{5 mm BBO BB-1H Z-GRD Z8007/0096 } & & Date & $18 \operatorname{Jan} 2019$ 08:34:56 \\
\hline Date Stamp & \multicolumn{3}{|c|}{18 Jan 2019 08:34:56 } & File Name & \multicolumn{3}{|c|}{ C:IUSERSIKARIYIDESKTOPINMRILSK-5-16I2IFID } \\
\hline Frequency $(\mathrm{MHz})$ & 125.76 & Nucleus & $13 \mathrm{C}$ & Number of Transients & & Origin & spect \\
\hline Original Points Count & 32768 & Owner & Hartley & Points Count & 32768 & Pulse Sequence & zgpg30 \\
\hline Receiver Gain & 16384.00 & SW(cyclical) $(\mathrm{Hz})$ & 30303.03 & Solvent & \multicolumn{3}{|c|}{ DEUTERIUM OXIDE } \\
\hline Spectrum Offset (Hz) & 13902.9092 & Spectrum Type & STANDARD & Sweep Width $(\mathrm{Hz})$ & 30302.1 & \multicolumn{2}{|c|}{ Temperature (degree C) 25.000} \\
\hline
\end{tabular}

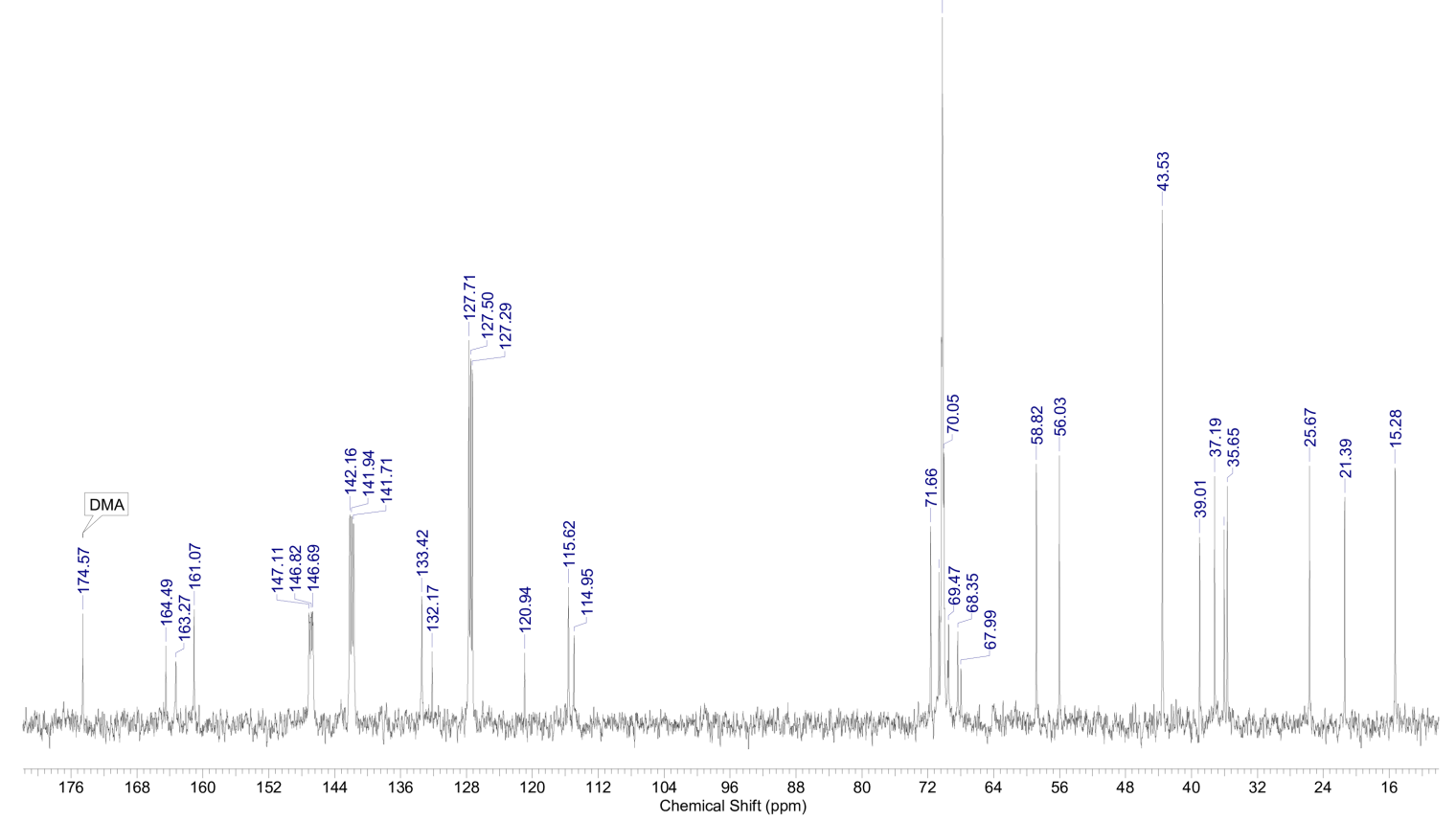

Figure S9. ${ }^{13} \mathrm{C}\left\{{ }^{1} \mathrm{H}\right\}$ NMR spectrum $\left(125 \mathrm{MHz}, \mathrm{D}_{2} \mathrm{O}\right)$ of the Ac1/An1 system after treatment with EDC, showing signals matching those of independently prepared An1 ( $\sim 50 \mathrm{mM} \mathrm{Ac1,} 0.5 \mathrm{M}$ pyridine- $d_{5}$ buffer, $\mathrm{pD} 4.5$, $\left.I=1.0 \mathrm{M}(\mathrm{NaCl}), \mathrm{D}_{2} \mathrm{O}, 298 \mathrm{~K}\right)$.

\section{Typical spectra}

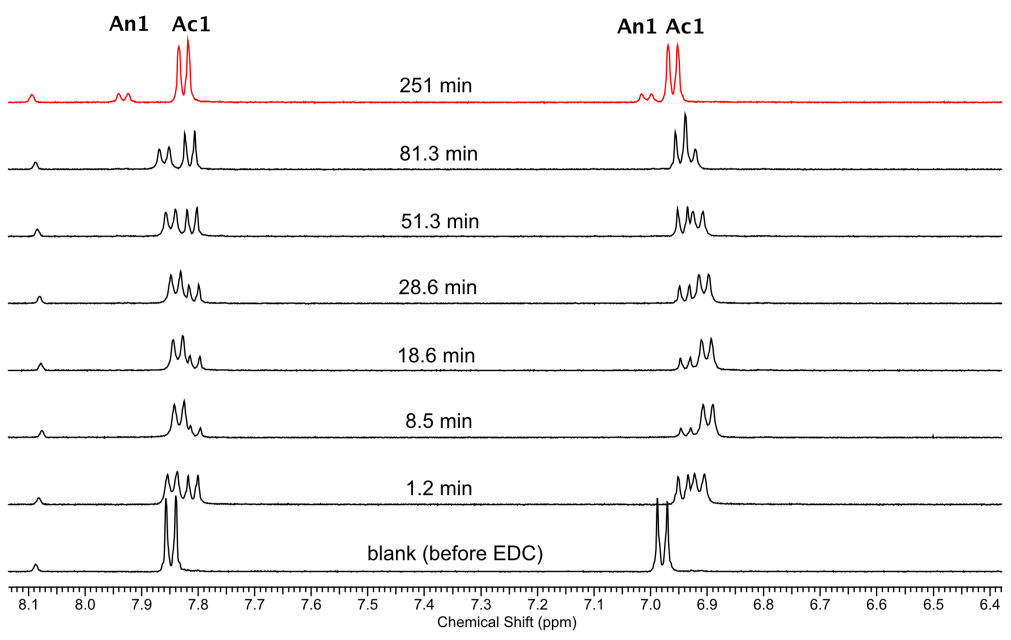

Figure S10. ${ }^{1} \mathrm{H}$ NMR spectra of An1 formation from Ac1 acquired during a typical kinetic run. 


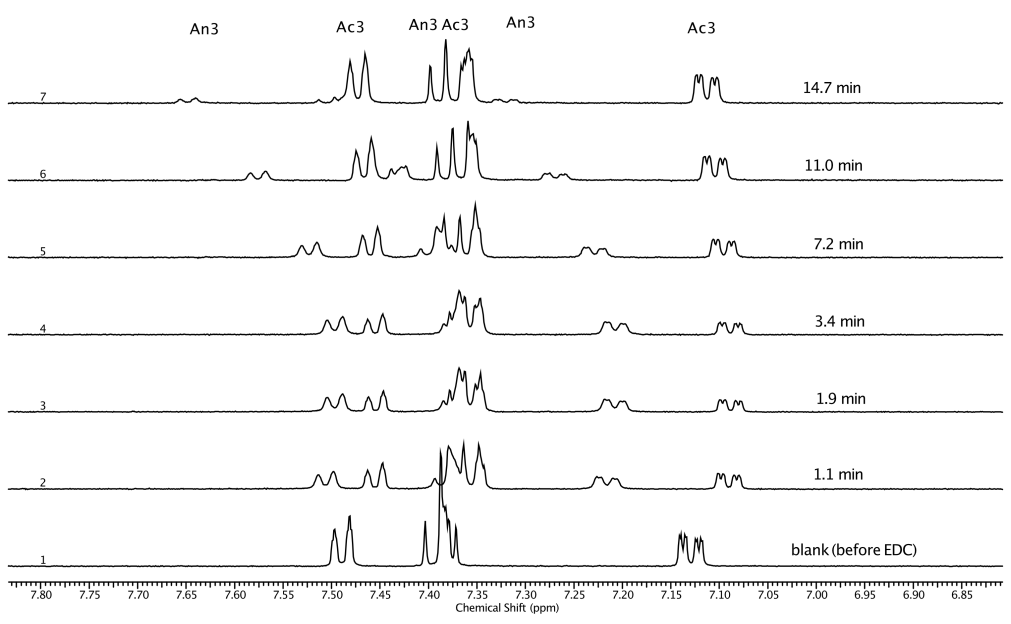

Figure S11. ${ }^{1} \mathrm{H}$ NMR spectra of An3 formation from Ac3 acquired during a typical kinetic run.

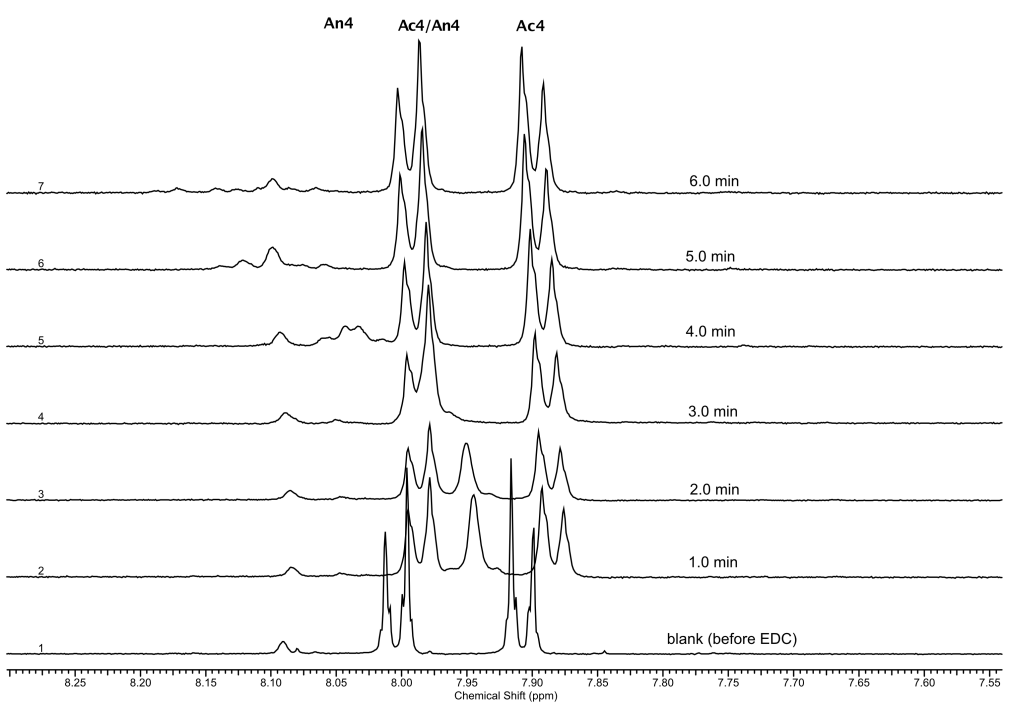

Figure S12. ${ }^{1} \mathrm{H}$ NMR spectra of An4 formation from Ac4 acquired during a typical kinetic run.

\section{Fitting}

As discussed in the manuscript, the kinetic data was fit to the following mechanism:

$$
\begin{gathered}
\mathrm{Ac}+\mathrm{EDC} \stackrel{k_{1}}{\longrightarrow} \mathrm{Int}+\mathrm{EDU} \\
\mathrm{Int}+\mathrm{Ac} \underset{k_{-2}}{\stackrel{k_{2}}{\rightleftharpoons}} \mathrm{An} \\
\mathrm{Int} \stackrel{k_{3}}{\longrightarrow} \mathrm{Ac} \\
\mathrm{EDC} \stackrel{k_{4}}{\longrightarrow} \mathrm{EDU}
\end{gathered}
$$

where Ac is the acid, An is the anhydride, and Int is the acylpyridinium intermediate. The system is therefore described by the following set of differential equations: 


$$
\begin{aligned}
\frac{\mathrm{d}[\mathrm{Ac}]}{\mathrm{d} t} & =-k_{1}[\mathrm{Ac}][\mathrm{EDC}]-k_{2}[\mathrm{Int}][\mathrm{Ac}]+k_{-2}[\mathrm{An}]+k_{3}[\mathrm{Int}] \\
\frac{\mathrm{d}[\mathrm{An}]}{\mathrm{d} t} & =+k_{2}[\mathrm{Int}][\mathrm{Ac}]-k_{-2}[\mathrm{An}] \\
\frac{\mathrm{d}[\mathrm{EDC}]}{\mathrm{d} t} & =-k_{1}[\mathrm{Ac}][\mathrm{EDC}]-k_{4}[\mathrm{EDC}] \\
\frac{\mathrm{d}[\mathrm{EDU}]}{\mathrm{d} t} & =+k_{1}[\mathrm{Ac}][\mathrm{EDC}]+k_{4}[\mathrm{EDC}] \\
\frac{\mathrm{d}[\mathrm{Int}]}{\mathrm{d} t} & =+k_{1}[\mathrm{Ac}][\mathrm{EDC}]-k_{2}[\mathrm{Int}][\mathrm{Ac}]+k_{-2}[\mathrm{An}]-k_{3}[\mathrm{Int}]
\end{aligned}
$$

We assume a steady-state in $[\operatorname{Int}]\left(\frac{\mathrm{d}[\mathrm{Int}]}{\mathrm{d} t}=0\right)$, which gives:

$$
[\operatorname{Int}]=\frac{k_{1}[\mathrm{Ac}][\mathrm{EDC}]}{k_{2}[\mathrm{Ac}]+k_{3}}+\frac{k_{-2}[\mathrm{An}]}{k_{2}[\mathrm{Ac}]+k_{3}}
$$

Substitution back into eqs 6 and 7 yields the following:

$$
\begin{aligned}
& \frac{\mathrm{d}[\mathrm{Ac}]}{\mathrm{d} t}=-k_{1}[\mathrm{Ac}][\mathrm{EDC}]-\frac{k_{1}[\mathrm{Ac}]^{2}[\mathrm{EDC}]}{[\mathrm{Ac}]+\alpha}-\frac{k_{-2}[\mathrm{An}][\mathrm{Ac}]}{[\mathrm{Ac}]+\alpha}+k_{-2}[\mathrm{An}]+\frac{k_{1} \alpha[\mathrm{Ac}][\mathrm{EDC}]}{[\mathrm{Ac}]+\alpha}+\frac{k_{-2} \alpha[\mathrm{An}]}{[\mathrm{Ac}]+\alpha} \\
& \frac{\mathrm{d}[\mathrm{An}]}{\mathrm{d} t}=-k_{-2}[\mathrm{An}]+\frac{k_{1}[\mathrm{Ac}]^{2}[\mathrm{EDC}]}{[\mathrm{Ac}]+\alpha}+\frac{k_{-2}[\mathrm{An}][\mathrm{Ac}]}{[\mathrm{Ac}]+\alpha}
\end{aligned}
$$

where $\alpha=k_{3} / k_{2}$.

The equations for the net anhydride generated in each experiment (eq 5 in the manuscript) are obtained by integrating eq 13 . Since $[\mathrm{An}]$ is the same (0) at the beginning and end of each experiment, $\int_{0}^{\infty}[\mathrm{An}] \mathrm{d} t=0$. Thus,

$$
\begin{aligned}
\int_{0}^{\infty}-k_{-2}[\mathrm{An}]+\frac{k_{1}[\mathrm{Ac}]^{2}[\mathrm{EDC}]}{[\mathrm{Ac}]+\alpha}+\frac{k_{-2}[\mathrm{An}][\mathrm{Ac}]}{[\mathrm{Ac}]+\alpha} \mathrm{d} t & =0 \\
\int_{0}^{\infty} k_{-2}[\mathrm{An}]-\frac{k_{-2}[\mathrm{An}][\mathrm{Ac}]}{[\mathrm{Ac}]+\alpha} \mathrm{d} t & =\int_{0}^{\infty} \frac{k_{1}[\mathrm{Ac}]^{2}[\mathrm{EDC}]}{[\mathrm{Ac}]+\alpha} \mathrm{d} t \\
\int_{0}^{\infty} k_{-2}[\mathrm{An}]\left(1-\frac{[\mathrm{Ac}]}{[\mathrm{Ac}]+\alpha}\right) \mathrm{d} t & =\int_{0}^{\infty} k_{1}[\mathrm{Ac}][\mathrm{EDC}]\left(1-\frac{\alpha}{[\mathrm{Ac}]+\alpha}\right) \mathrm{d} t
\end{aligned}
$$

The integral on the left of eq 16 represents the total anhydride destroyed; $\int_{0}^{\infty} k_{-2}[\mathrm{An}] \mathrm{d} t$ would be the total flux through the $k_{-2}$ reaction (eq 3), with $\frac{[\mathrm{Ac}]}{[\mathrm{Ac}]+\alpha}$ correcting for the anhydride that is regenerated $\left(k_{2}\right)$ instead of undergoing hydrolysis $\left(k_{3}\right)$. Similarly, the integral on the right represents the total anhydride generated from EDC; $\int_{0}^{\infty} k_{1}[\mathrm{Ac}][\mathrm{EDC}] \mathrm{d} t$ would be the total flux through the $k_{1}$ reaction, with $\frac{\alpha}{[\mathrm{Ac}]+\alpha}$ correcting for the intermediate generated that undergoes hydrolysis instead of giving anhydride.

The system of equations (eqs 8, 9,12, and 13) was solved numerically for both the fits to the experimental data (the contribution of eq 5 was omitted when evaluating the systems with different pD's or buffer concentrations ${ }^{a}$ ) and the simulations. This was done using a custom program written in Python 3 ("kinmodel") that is included as a separate file. The compressed .tar.gz file can be installed directly using Python's pip package manager, or the code inspected and used after decompression.

The program uses standard capabilities from the Python packages NumPy and SciPy for the actual solving of the equations and regression. It implements and automates some useful features for this work:

${ }^{a}$ The contribution of eq 5 was found to be quite small under the standard conditions, and has only a negligible effect on the extracted parameters. 
- New kinetic models can be added very easily. Note that it does not interpret the chemistry itself: it must be provided with a system of differential equations such as eqs $8,9,12$, and 13.

- These same model input files are used for both fitting and simulation.

- A custom error function is used, so that the errors on the concentrations (c), which are derived from NMR measurements, can be properly weighted by $1 / c$. We set an upper limit on these weights of $(5 \mathrm{mM})^{-1}$, a conservative value derived from integrating the noise in a blank region of a typical spectrum. ${ }^{3}$

- Quantities of interest, such as yields or peak concentrations, are automatically calculated for both experimental fits and simulations (provided they have been specified in the model being used).

- Standard errors on the regression, obtained using a non-parametric random-x bootstrapping method, are automatically calculated. These estimates are derived from repeating the fits many times to new datasets obtained by randomly choosing from the experimental data points. ${ }^{4}$ The $t=0$ points were always included in these new datasets since this point is separately measured for each system and not unknown. In this work, 10,000 bootstrapping iterations were performed in order to estimate the errors.

- Confidence contour maps can be generated to explore the response of the error function to variations in the fitted parameters (discussed further below). ${ }^{5}$

- Large numbers of simulations are easily executed, allowing the dependence of various properties on changing parameters and conditions to be determined (e.g., to generate the dependence of yield on [EDC]).

As noted in the manuscript, care was taken to ensure that enough data had been collected to adequately determine the fit parameters. This was particularly important for $k_{-2}$ and $\alpha$, which are negatively correlated. The quality of the estimates of these two parameters was verified in two ways: (1) by examining the uncertainties in the parameters derived from the bootstrapping method described above, and (2) by looking at confidence contour maps for pairwise comparisons of the parameters.

For example, fitting to a single dataset for Ac2 yields the plot shown in Figure S13. The overall quality of the fit is excellent and the $95 \%$ confidence intervals for the concentrations are in good agreement with the data. However, the uncertainty in the parameters, listed in Table S1, is very large. While $k_{1}$ is well-constrained by the data, the confidence intervals for $k_{-2}$ and $\alpha$ each span three orders of magnitude. This issue is important for the Ac2, Ac3, and Ac4 systems. For Ac1, the time scales of anhydride formation and hydrolysis are sufficiently different that all three parameters could be determined from a single dataset, but the fit was still done globally to the full set of multiple kinetic runs for consistency. 

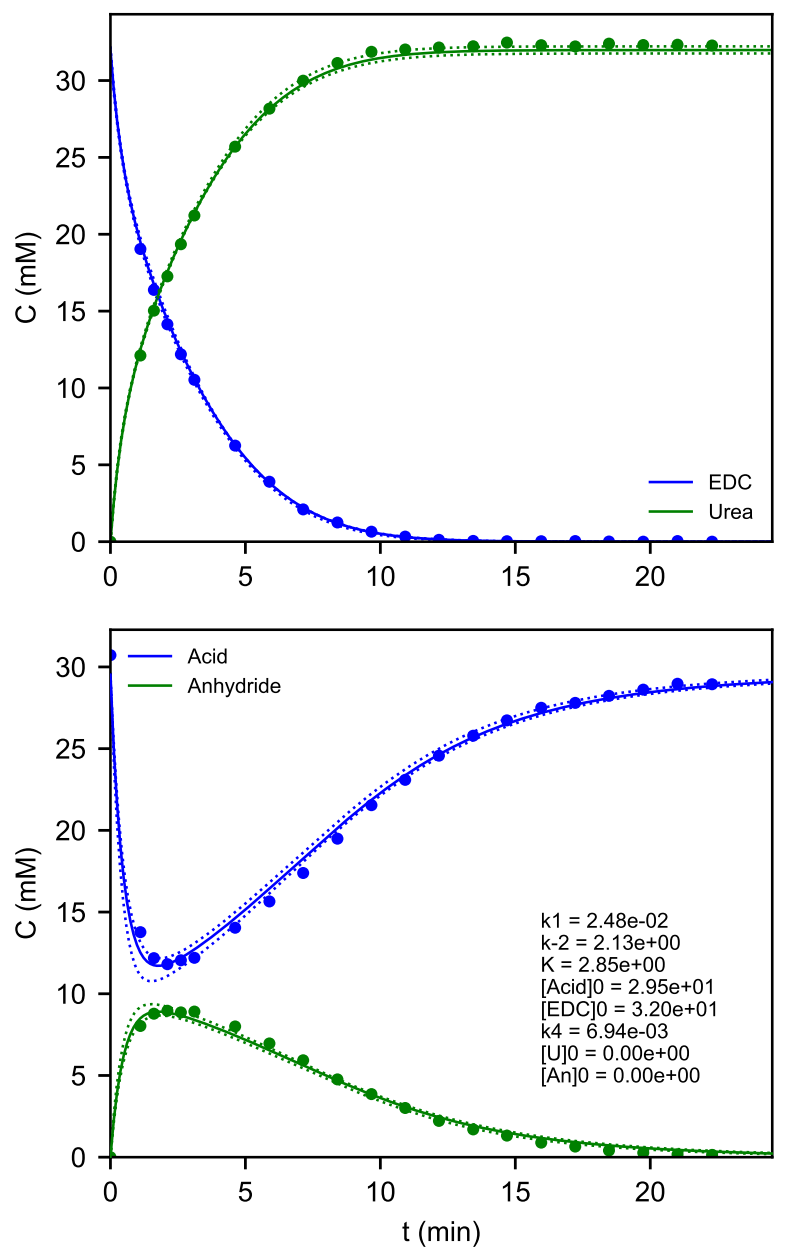

Figure S13. Kinetic run for Ac2, fitting the mechanism to this single dataset. The dashed lines represent $95 \%$ confidence intervals for the fits ( $K$ in the figure is $\alpha$ ).

\begin{tabular}{llc}
\hline Parameter & \multicolumn{1}{c}{ Optimized value } & $95 \%$ confidence interval \\
\hline$k_{1}$ & $2.48 \times 10^{-2} \mathrm{mM}^{-1} \mathrm{~min}^{-1}$ & $\left(2.40 \times 10^{-2}, 2.64 \times 10^{-2}\right)$ \\
$k_{-2}$ & $2.13 \mathrm{~min}^{-1}$ & $\left(1.51,1.30 \times 10^{3}\right)$ \\
$\alpha$ & $2.85 \mathrm{mM}$ & $\left(4.43 \times 10^{-3}, 4.07\right)$ \\
\hline
\end{tabular}

Table S1. Optimized parameters and confidence intervals obtained from the data in Figure S13.

These confidence intervals are narrowed considerably when additional kinetic runs are added to the fits. Once acceptable uncertainties were obtained, the interdependence of the parameters was further examined by constructing heat maps of the total error associated with the regression as a function of each pair of parameters. ${ }^{5}$ The normalized inverse of the error is plotted; thus, the optimum values will give maxima in the plots.

If the two parameters are uncorrelated and well-constrained by the data, the contour plot will show a single symmetrical peak centered on the optimized values of the parameters. This is true, for example, for the parameters $k_{1}$ and $\alpha$, as in Figure S16 below. If the two parameters are correlated, the shape of the peak will be distorted to reflect the correlation. This is true of $k_{-2}$ and $\alpha$, as in Figure S15. Poorly constrained parameters would yield plots with nearly equal values of the error function over wide ranges of parameter values instead of distinct maxima.

These plots confirm that the data are sufficient to experimentally determine the parameters of interest. However, the Ac4/An4 system is approaching the limits of the experiments used here, as shown by both the uncertainties and the confidence contour plots. For systems that undergo faster anhydride hydrolysis it may be necessary to 
adopt a different experimental approach.

The fit_kinetics program provided by the kinmodel package includes help information that can be accessed with the command fit_kinetics -h once it has been installed. Typical processing of a dataset for this work would be carried out with the following command:

fit_kinetics model_name filename.csv -ks fixed_parameters -w 5 -bff -b 10000 -cci 40 -cy

where model_name is the model to be used (default models are listed in the help); filename.csv is the concentration vs time data as a single csv file; -ks fixed_parameters specifies kinetic parameters for the model that are not to be optimized (e.g., the EDC hydrolysis rate, with fixed_parameters replaced with the value of the rate constant); -w 5 specifies that relative errors are used with a floor of 5 concentration units; -b 10000 specifies 10,000 bootstrapping iterations; -cci 40 specifies the generation of the confidence contour plots and heat maps over a $40 \times 40$ grid; and -cy forces common $y$-axis limits across the generated plots a set of experiments (to facilitate comparison). Datasets are specified in the input csv file as follows: dataset title in line 1, column titles in line 2, conc vs time data in the following lines. The time must be the first column and, for the models used throughout the study, the concentrations are specified in the order anhydride, acid, EDU, EDC. Multiple datasets are included in a single input file with a blank line between them. The program outputs a text file with the regression output and a pdf figure of the plot for each dataset along with heat maps and contour plots for the parameter analysis.

\section{Standard conditions}

\section{Extracted parameters}

\begin{tabular}{llll}
\hline & $k_{1}\left(\times 10^{-2} \mathrm{mM}^{-1} \mathrm{~min}^{-1}\right)$ & $k_{-2}\left(\mathrm{~min}^{-1}\right)$ & $\alpha(\mathrm{mM})$ \\
\hline Ac1/An1 & $2.283_{-0.048}^{+0.039}$ & $0.0456_{-0.0024}^{+0.0029}$ & $6.33_{-0.31}^{+0.29}$ \\
Ac2/An2 & $2.497_{-0.032}^{+0.046}$ & $1.01_{-0.12}^{+0.20}$ & $5.26_{-0.89}^{+0.70}$ \\
Ac3/An3 & $2.169_{-0.025}^{+0.035}$ & $1.09_{-0.17}^{+0.26}$ & $6.6_{-1.2}^{+1.1}$ \\
Ac4/An4 & $1.817_{-0.030}^{+0.042}$ & $3.1_{-0.9}^{+3.0}$ & $14.5_{-7.3}^{+6.6}$ \\
\hline
\end{tabular}

Table S2. Experimental kinetic parameters for anhydride formation and hydrolysis ( $0.5 \mathrm{M}$ pyridine- $d_{5}$ buffer, pD 4.5, $\left.I=1.0 \mathrm{M}(\mathrm{NaCl}), \mathrm{D}_{2} \mathrm{O}, 298 \mathrm{~K}\right)$. 


\section{Kinetics experiments}
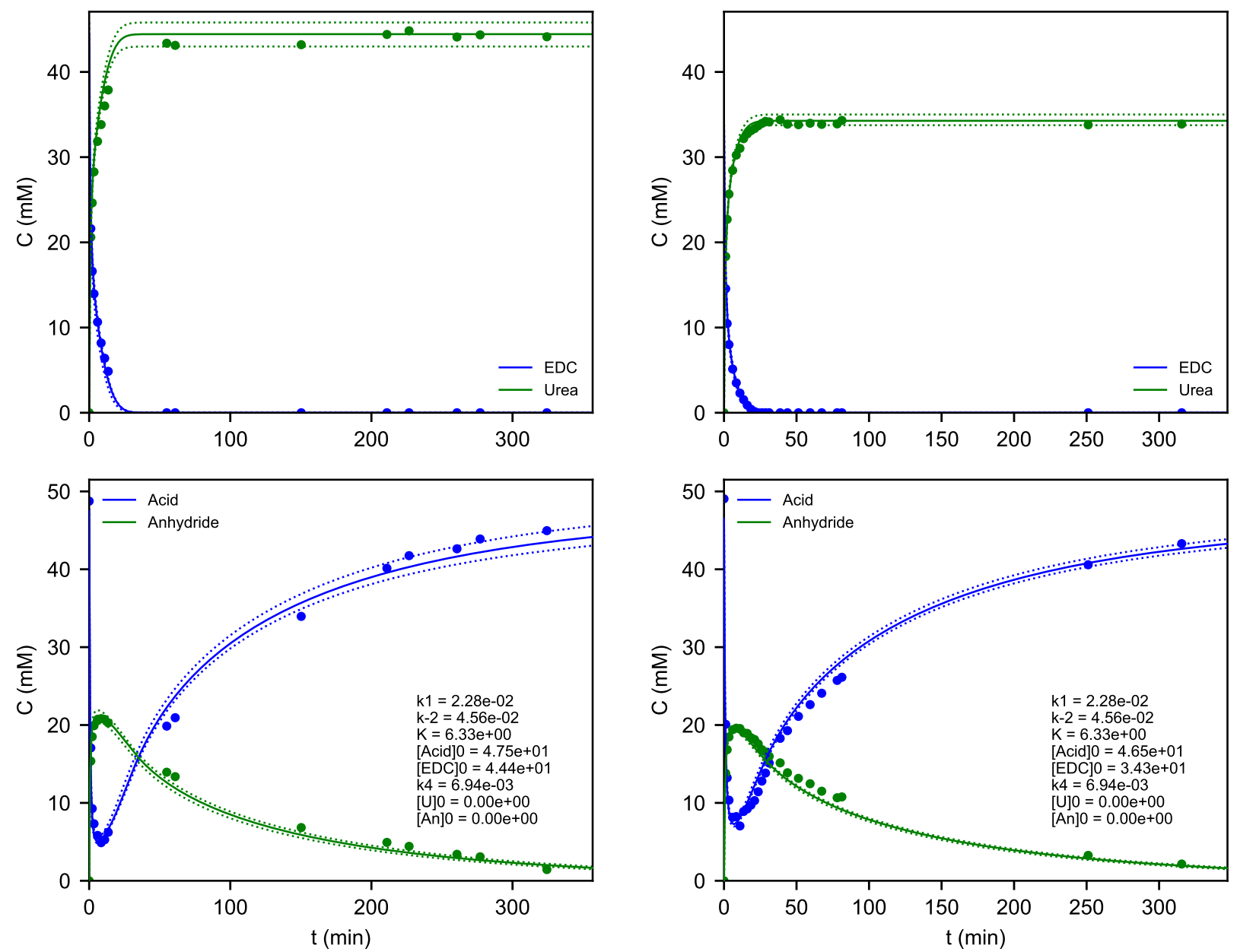

Figure S14. ${ }^{1} \mathrm{H}$ NMR spectroscopy monitoring of the treatment of Ac1 with EDC ( $0.5 \mathrm{M}$ pyridine- $d_{5}$ buffer, pD 4.5, $\left.I=1.0 \mathrm{M}(\mathrm{NaCl}), \mathrm{D}_{2} \mathrm{O}, 298 \mathrm{~K}\right)$. The dashed lines represent $95 \%$ confidence intervals for the fits $(K$ in the figure is $\alpha$ ) (continues on next page). 

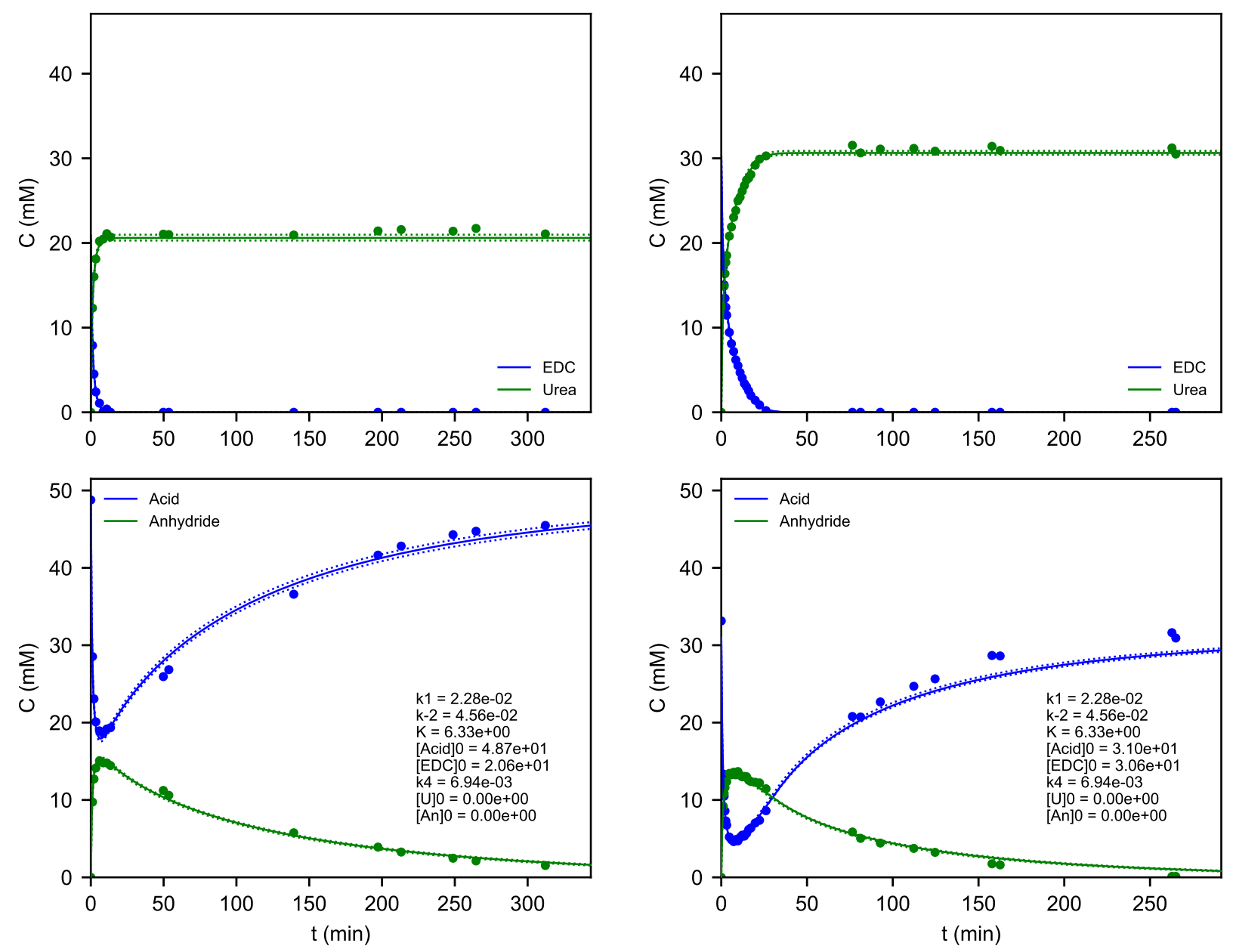

Figure S14. Continued. 

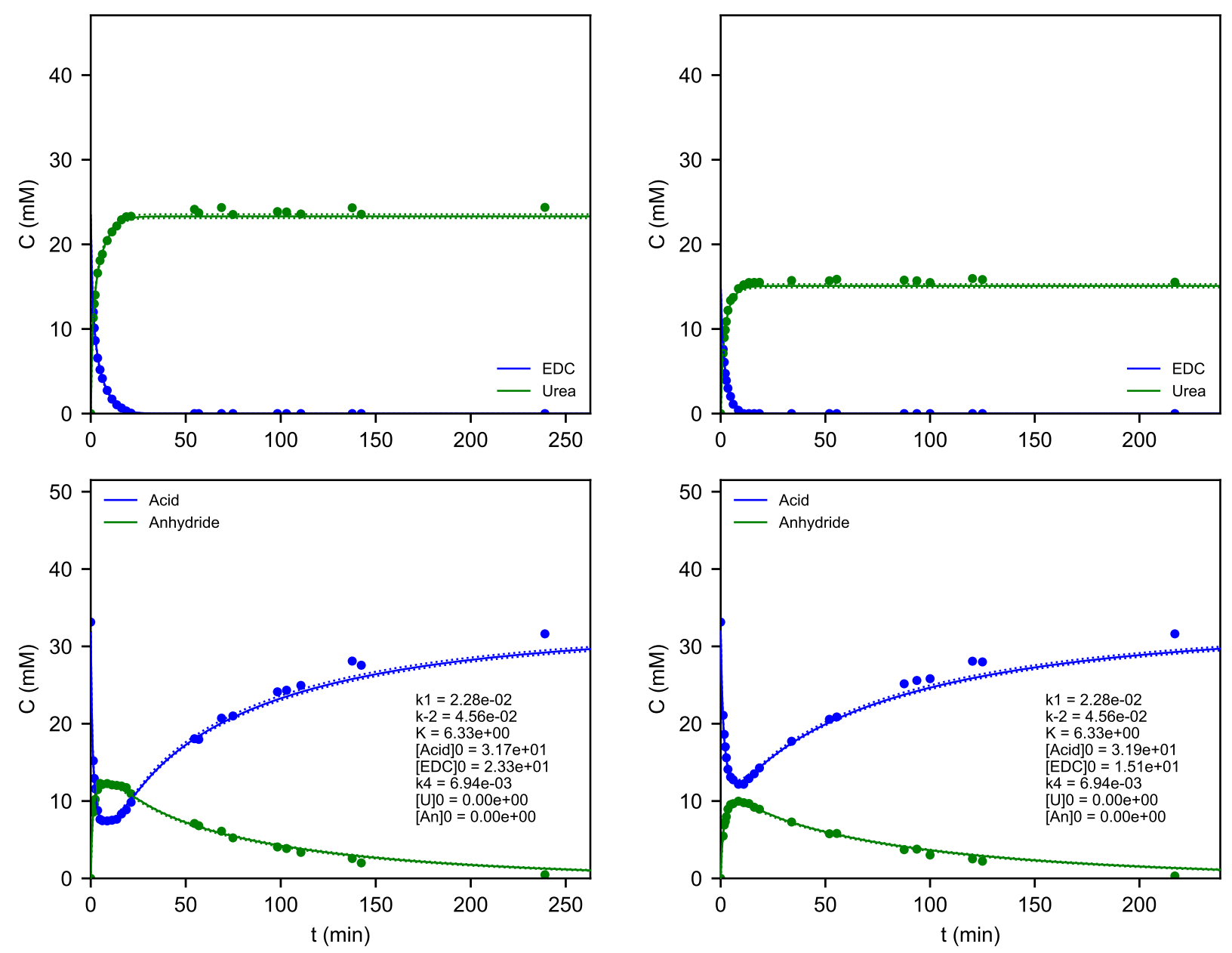

Figure S14. Continued. 

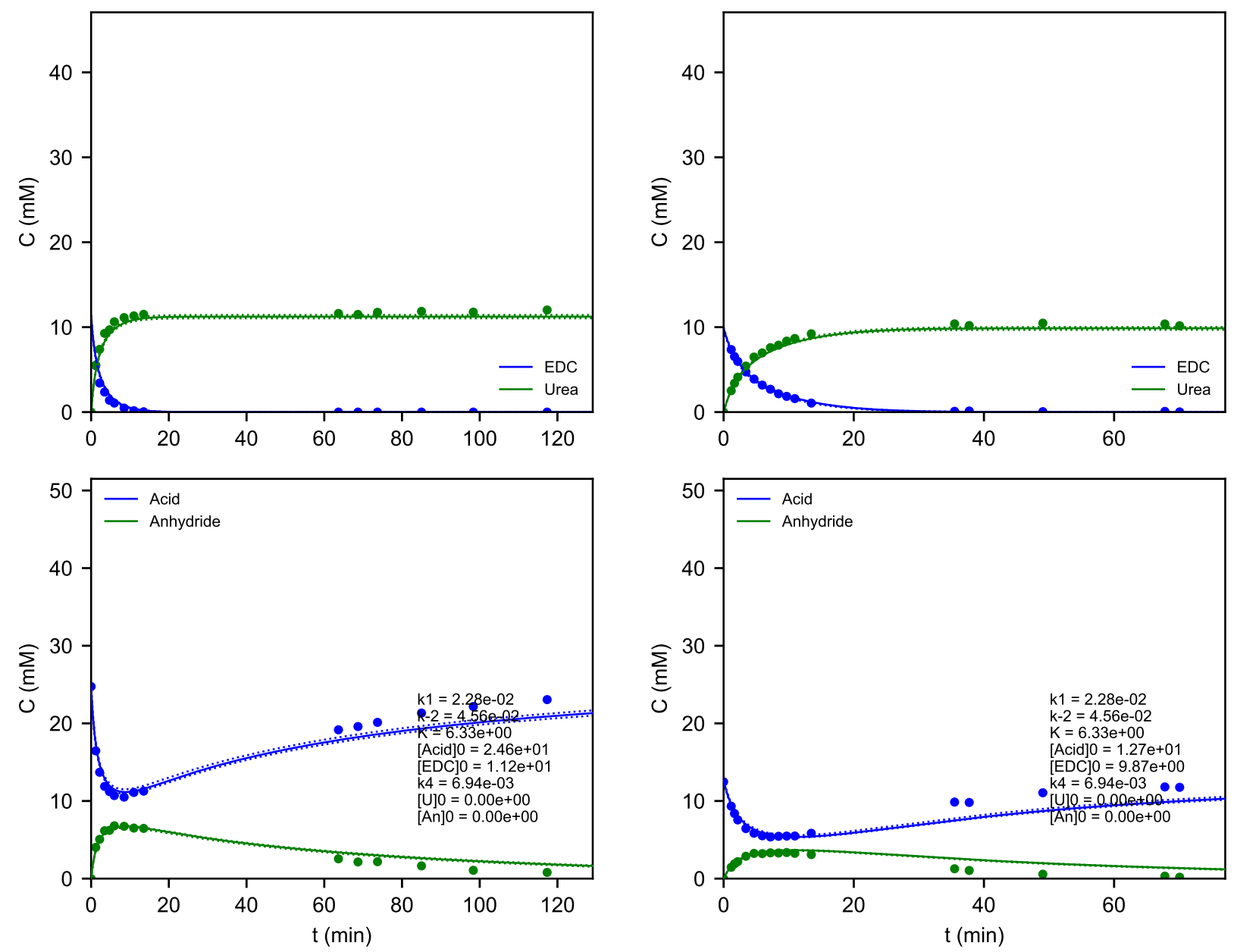

Figure S14. Continued.

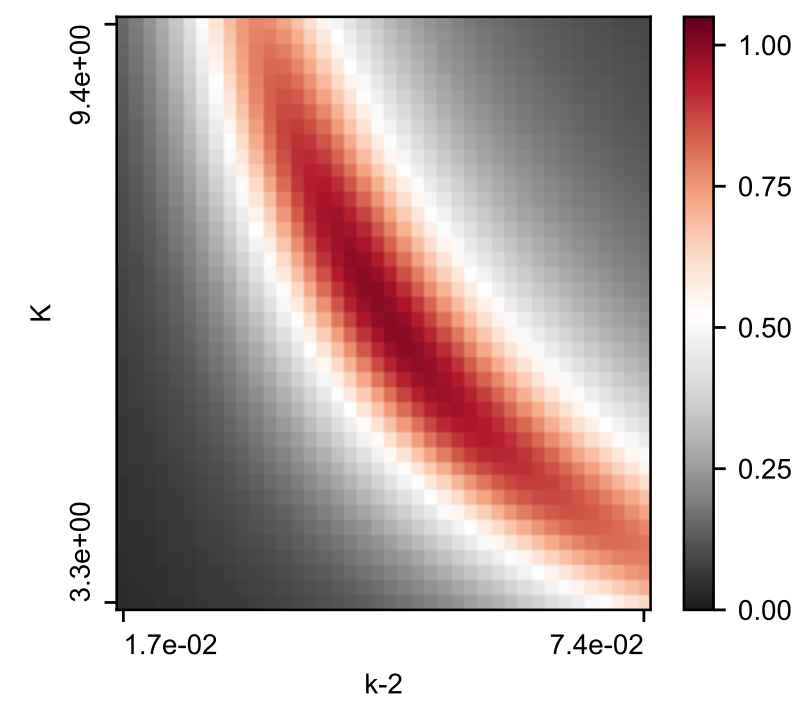

Figure S15. Heat map of the response of the regression error function to variation of $k_{-2}$ and $\alpha$ for the Ac1/An1 system ( $K$ in the figure is $\alpha$ ). The inverse error function is plotted, normalized against the value for the optimized system. 


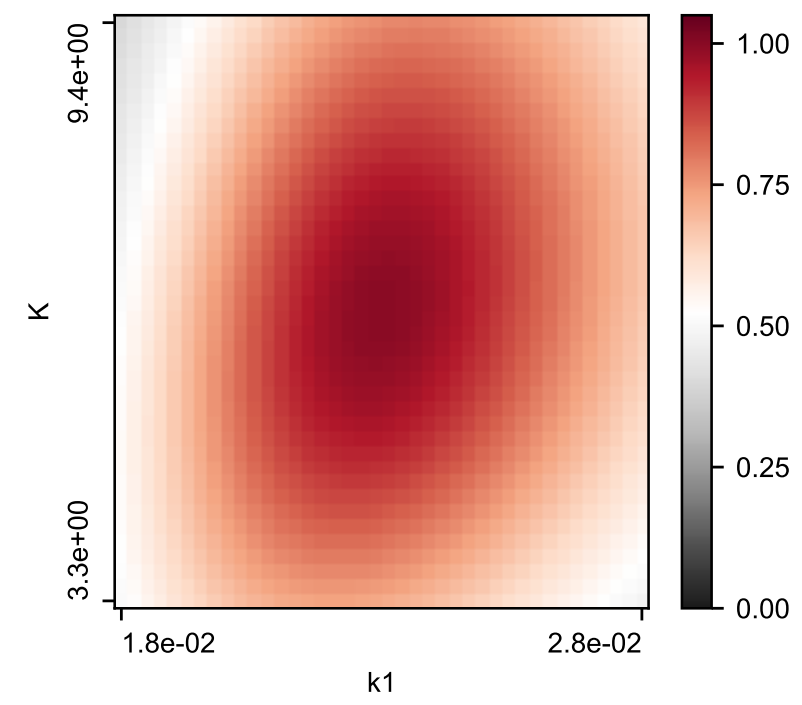

Figure S16. Heat map of the response of the regression error function to variation of $k_{1}$ and $\alpha$ for the Ac1/An1 system ( $K$ in the figure is $\alpha$ ). The inverse error function is plotted, normalized against the value for the optimized system.

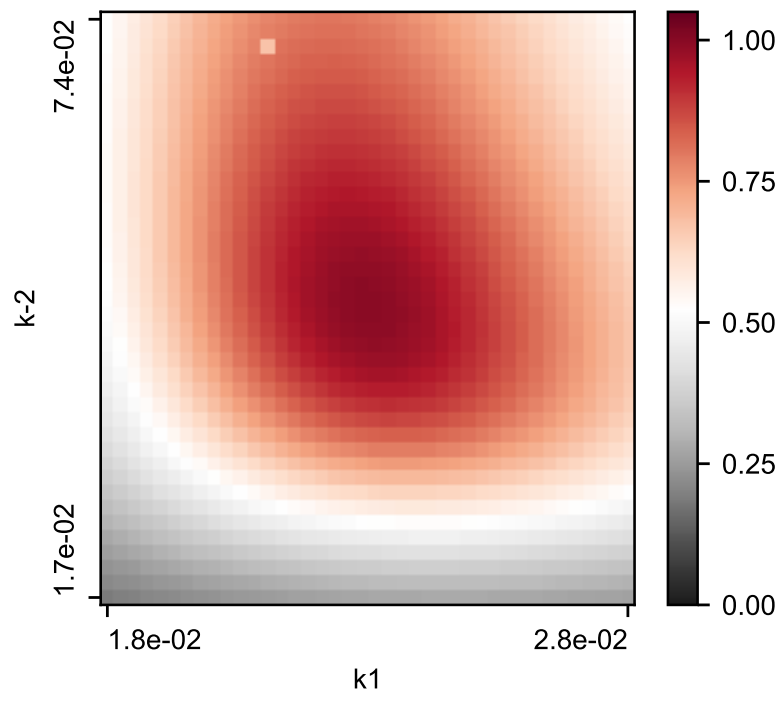

Figure S17. Heat map of the response of the regression error function to variation of $k_{1}$ and $k_{-2}$ for the Ac1/An1 system. The inverse error function is plotted, normalized against the value for the optimized system. 

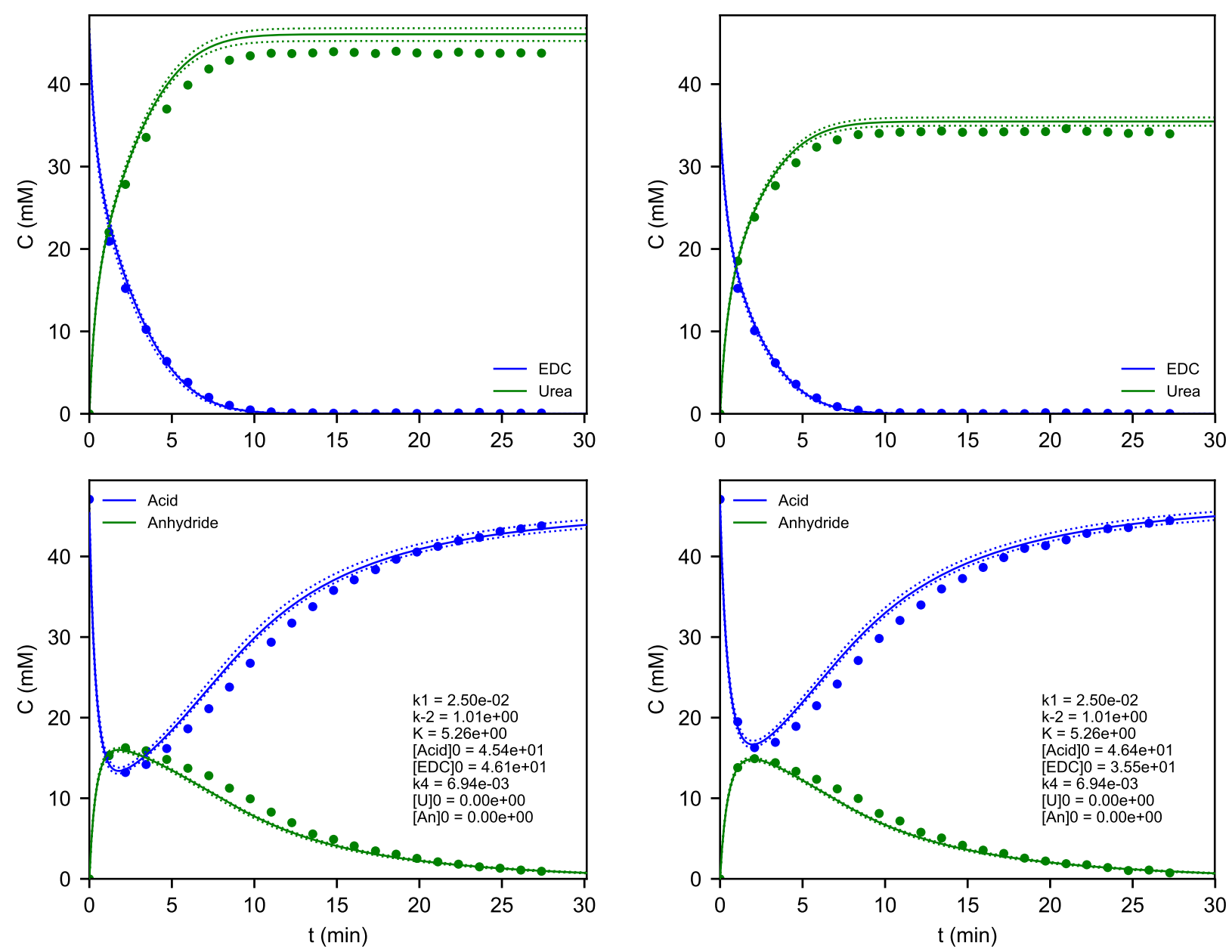

Figure S18. ${ }^{1} \mathrm{H}$ NMR spectroscopy monitoring of the treatment of Ac2 with EDC ( $0.5 \mathrm{M}$ pyridine- $d_{5}$ buffer, $\left.\mathrm{pD} 4.5, I=1.0 \mathrm{M}(\mathrm{NaCl}), \mathrm{D}_{2} \mathrm{O}, 298 \mathrm{~K}\right)$. The dashed lines represent $95 \%$ confidence intervals for the fits $(K$ in the figure is $\alpha$ ) (continues on next page). 

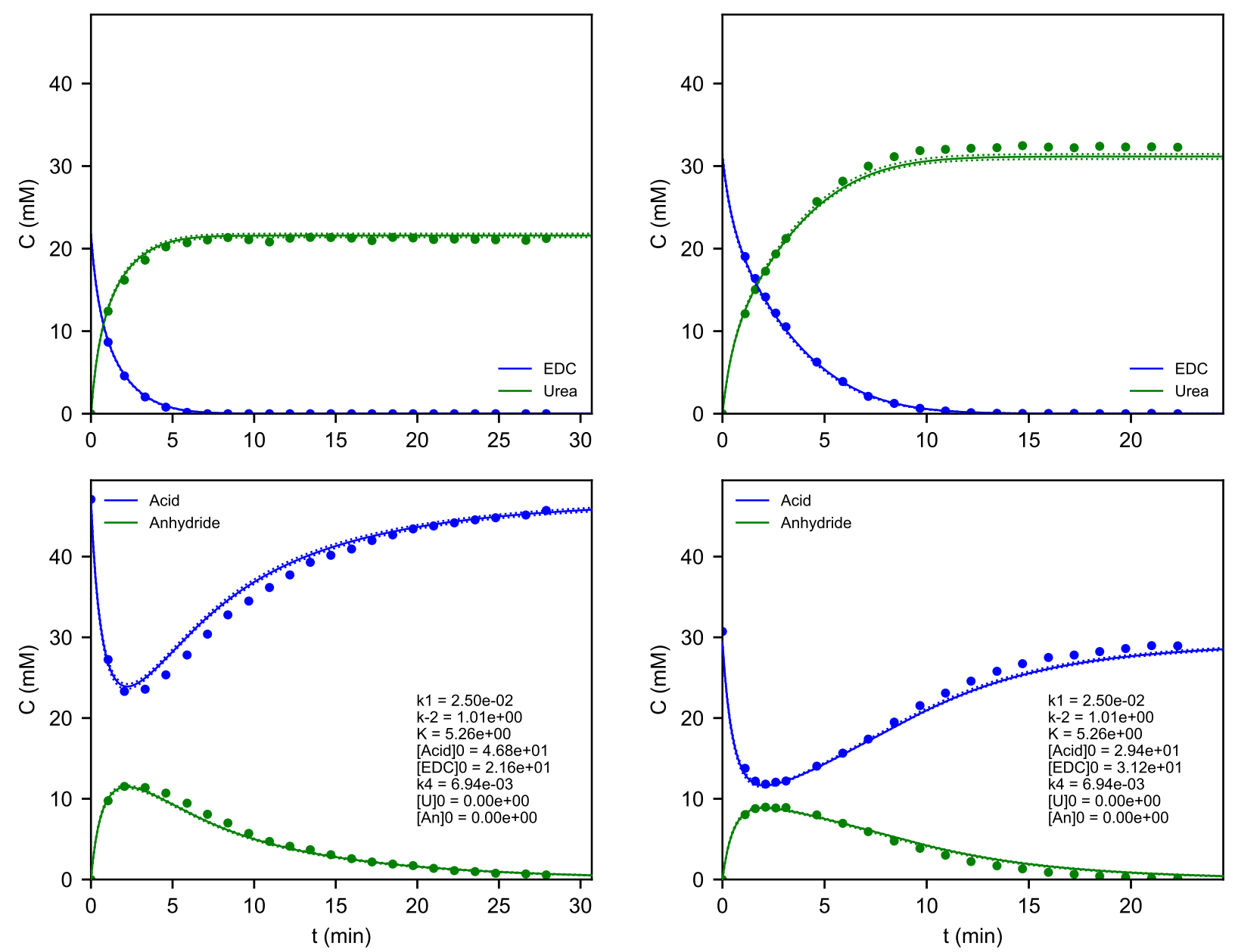

Figure S18. Continued. 

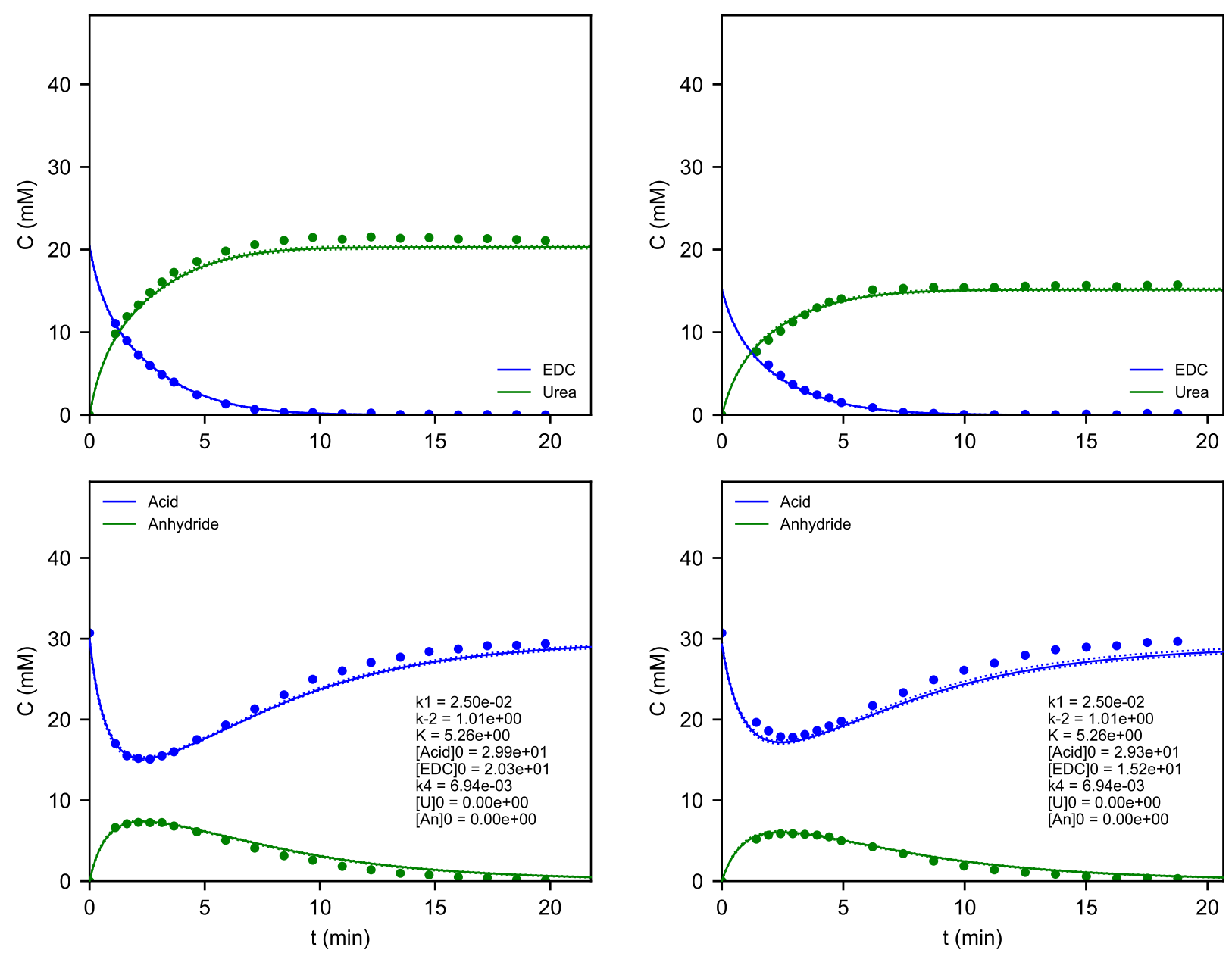

Figure S18. Continued. 

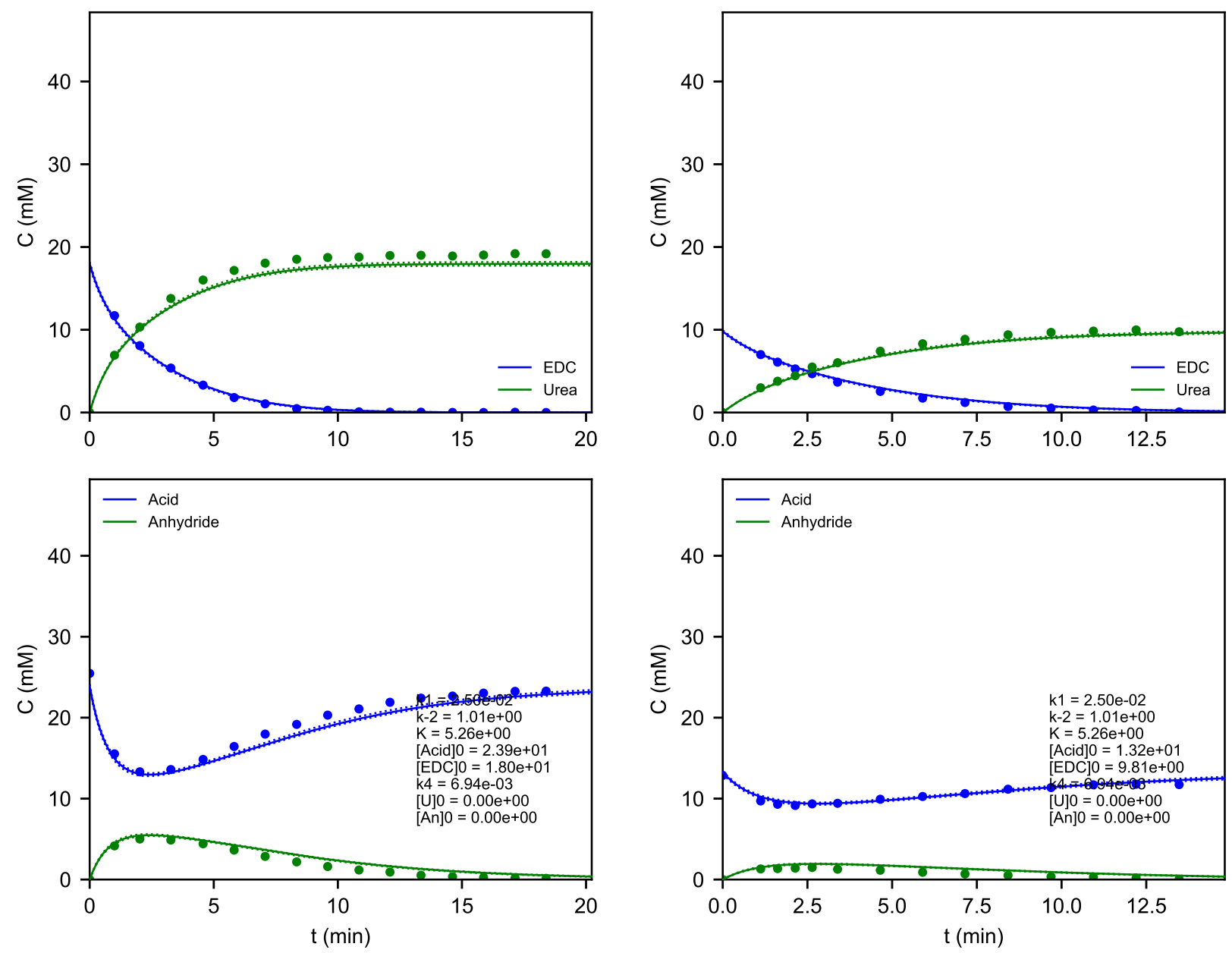

Figure S18. Continued.

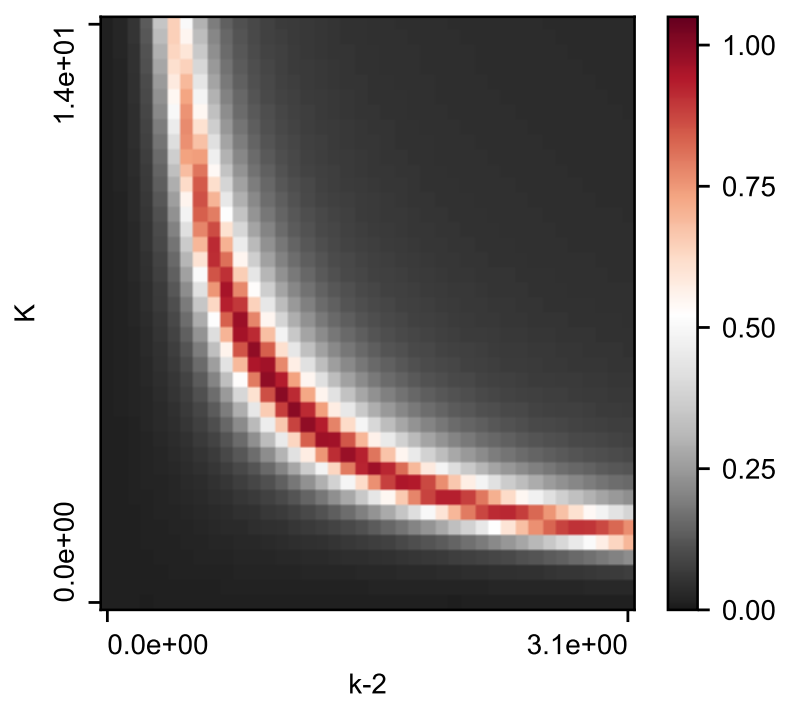

Figure S19. Heat map of the response of the regression error function to variation of $k_{-2}$ and $\alpha$ for the Ac2/An2 system ( $K$ in the figure is $\alpha$ ). The inverse error function is plotted, normalized against the value for the optimized system. 


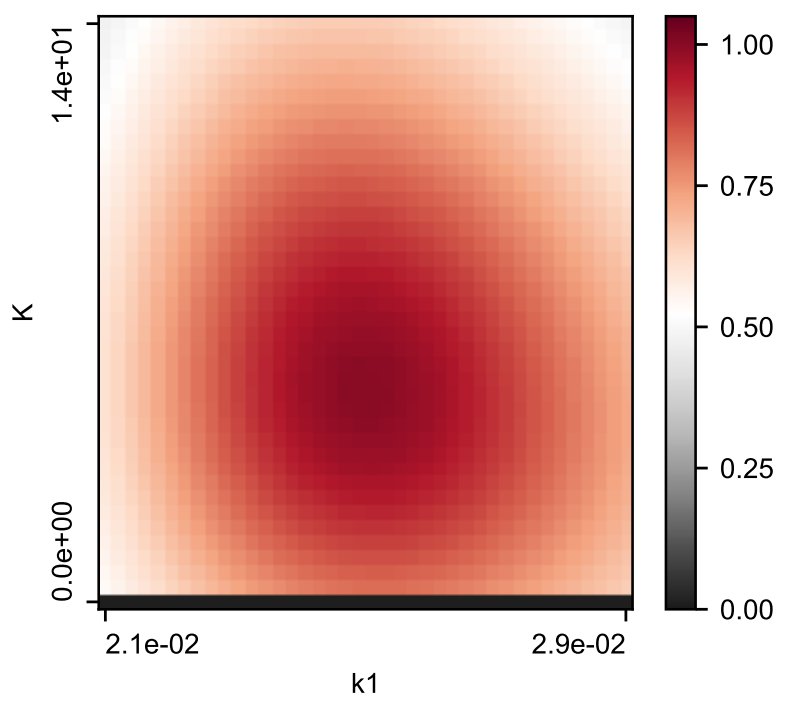

Figure S20. Heat map of the response of the regression error function to variation of $k_{1}$ and $\alpha$ for the Ac2/An2 system ( $K$ in the figure is $\alpha$ ). The inverse error function is plotted, normalized against the value for the optimized system.

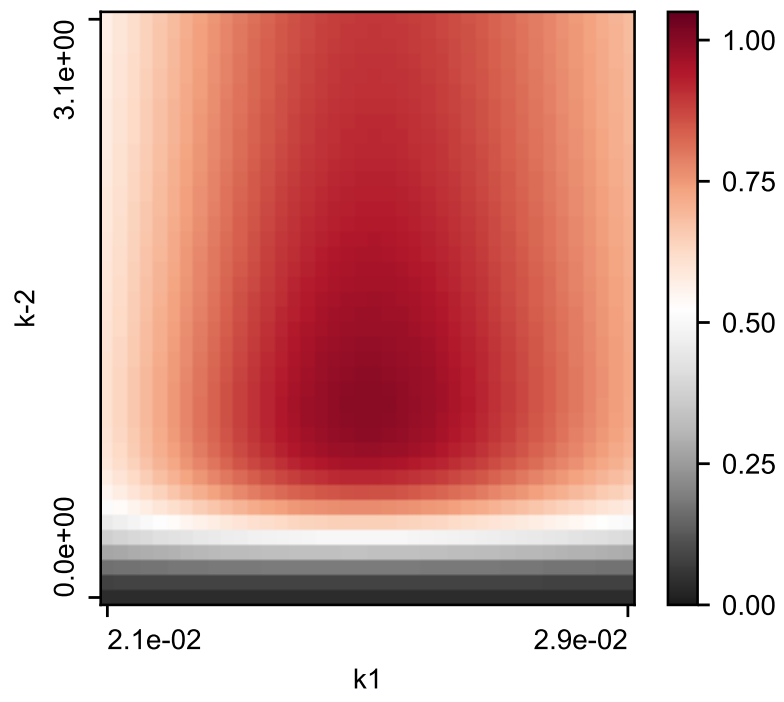

Figure S21. Heat map of the response of the regression error function to variation of $k_{1}$ and $k_{-2}$ for the Ac2/An2 system. The inverse error function is plotted, normalized against the value for the optimized system. 

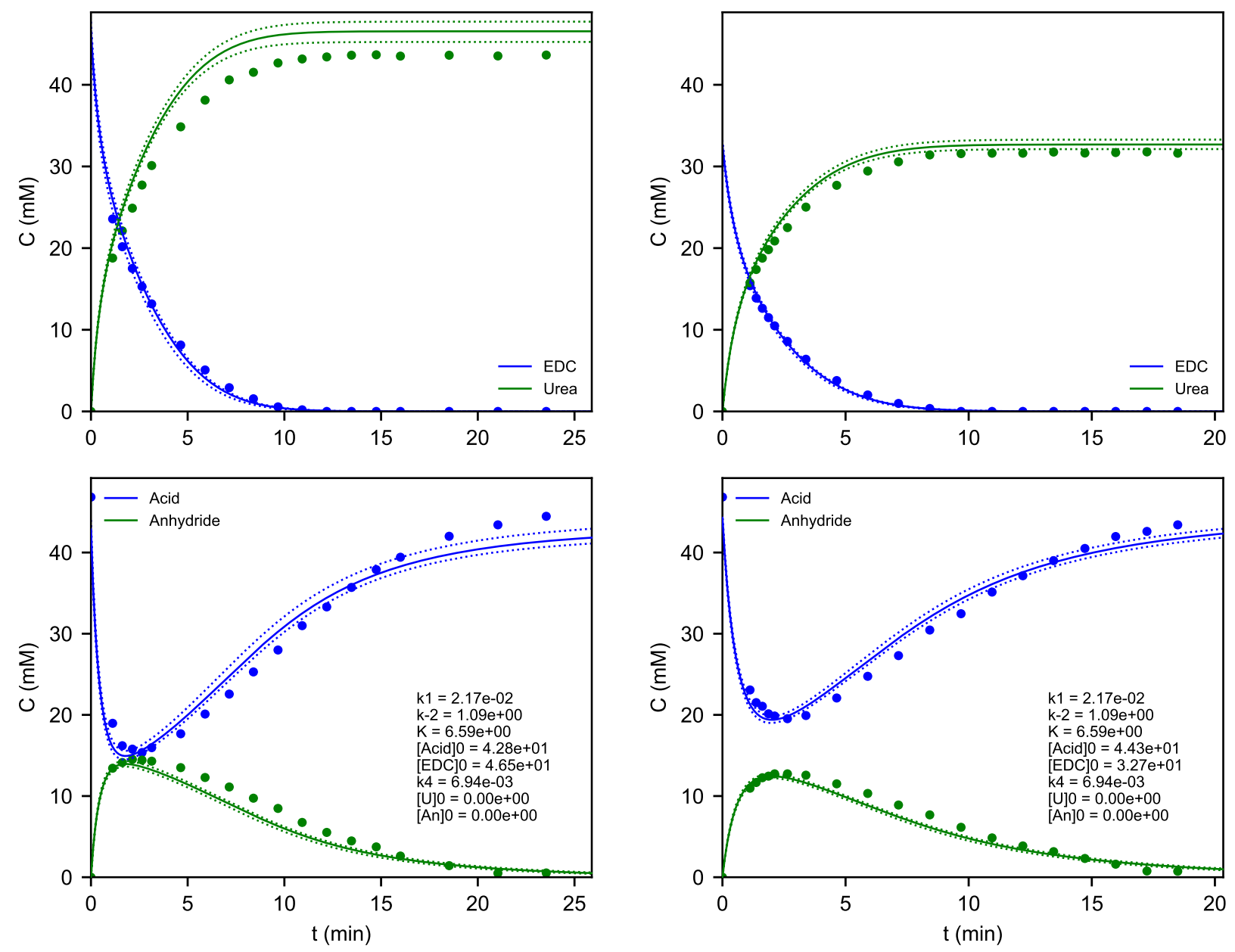

Figure S22. ${ }^{1} \mathrm{H}$ NMR spectroscopy monitoring of the treatment of Ac3 with EDC ( $0.5 \mathrm{M}$ pyridine- $d_{5}$ buffer, $\left.\mathrm{pD} 4.5, I=1.0 \mathrm{M}(\mathrm{NaCl}), \mathrm{D}_{2} \mathrm{O}, 298 \mathrm{~K}\right)$. The dashed lines represent $95 \%$ confidence intervals for the fits $(K$ in the figure is $\alpha$ ) (continues on next page). 

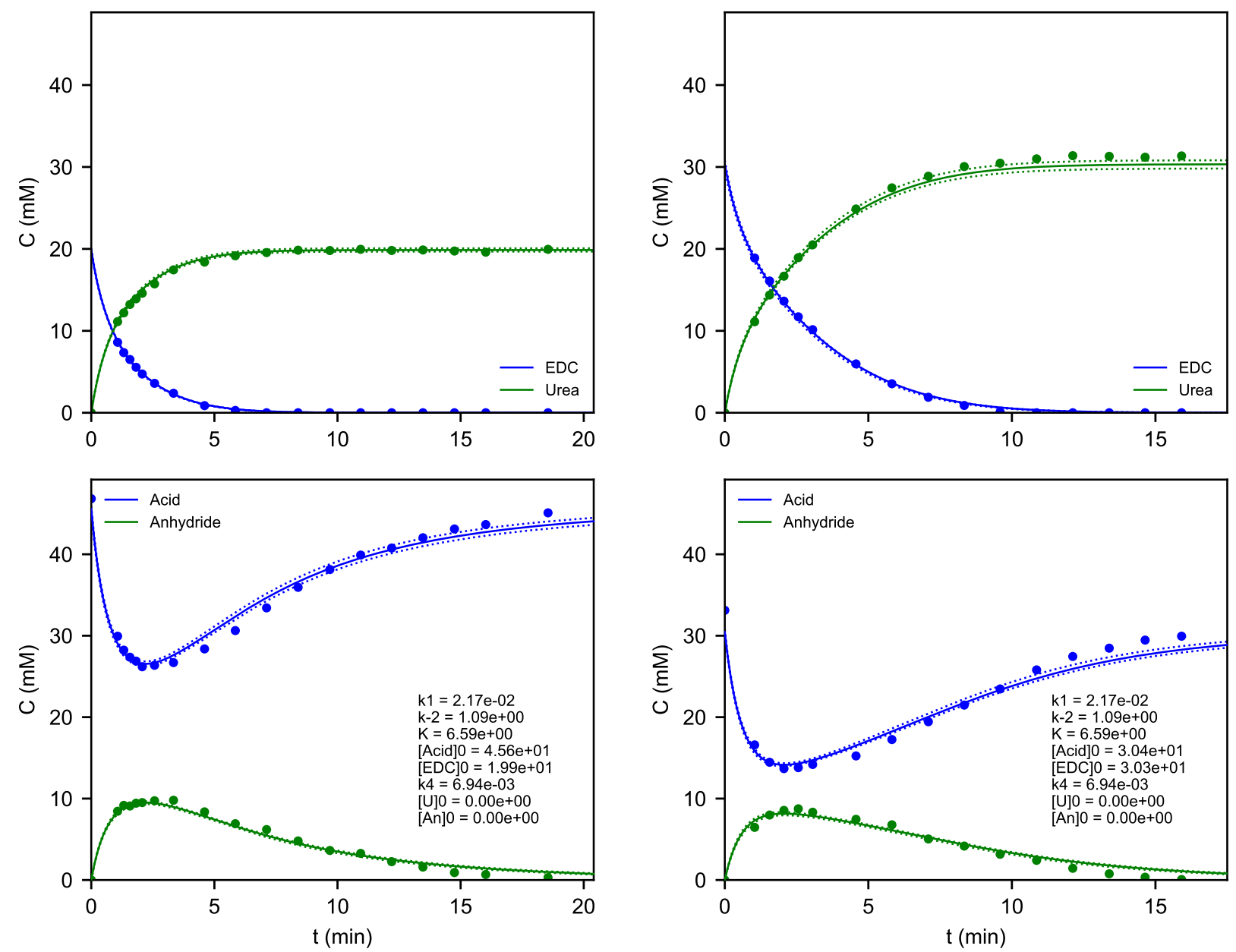

Figure S22. Continued. 

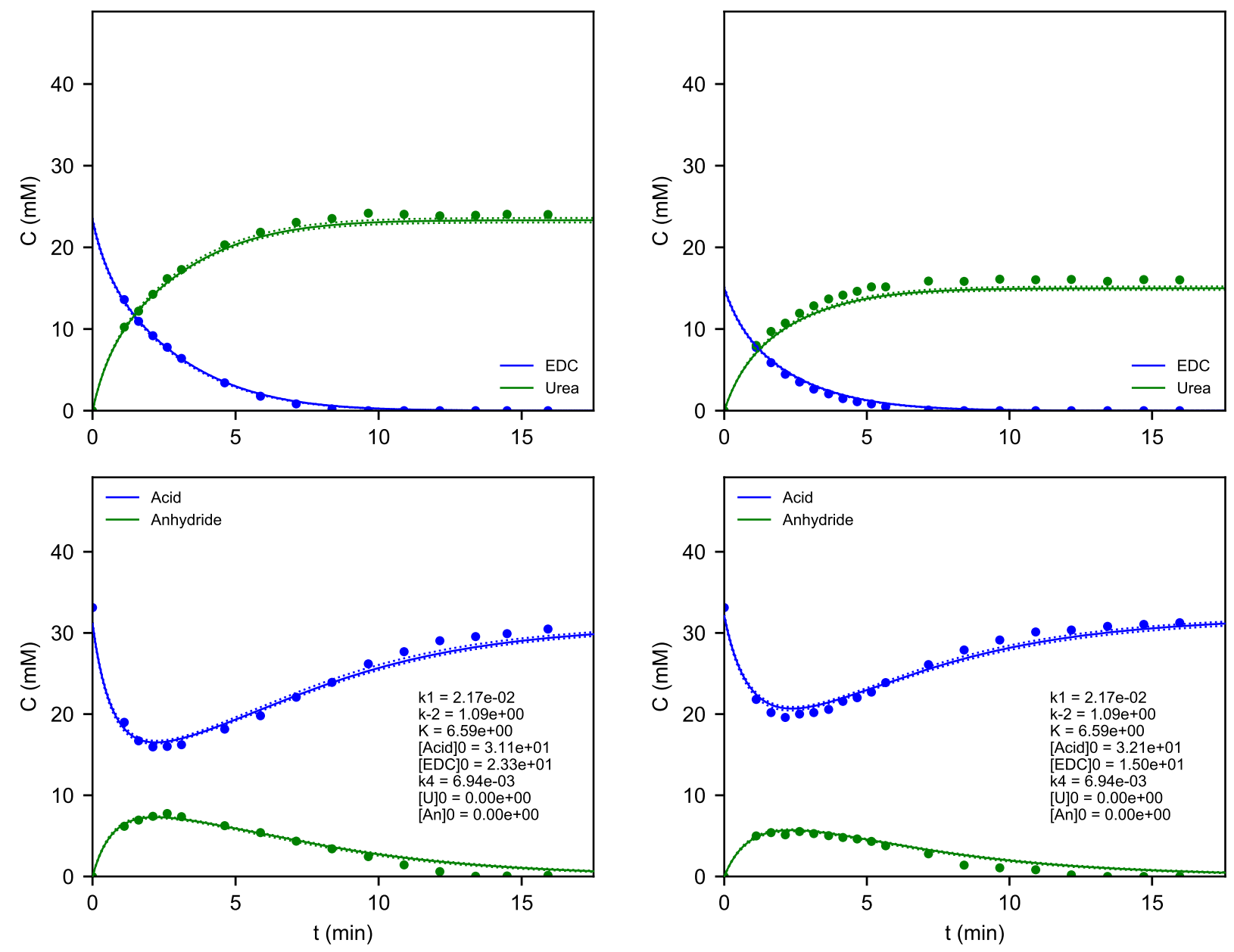

Figure S22. Continued. 

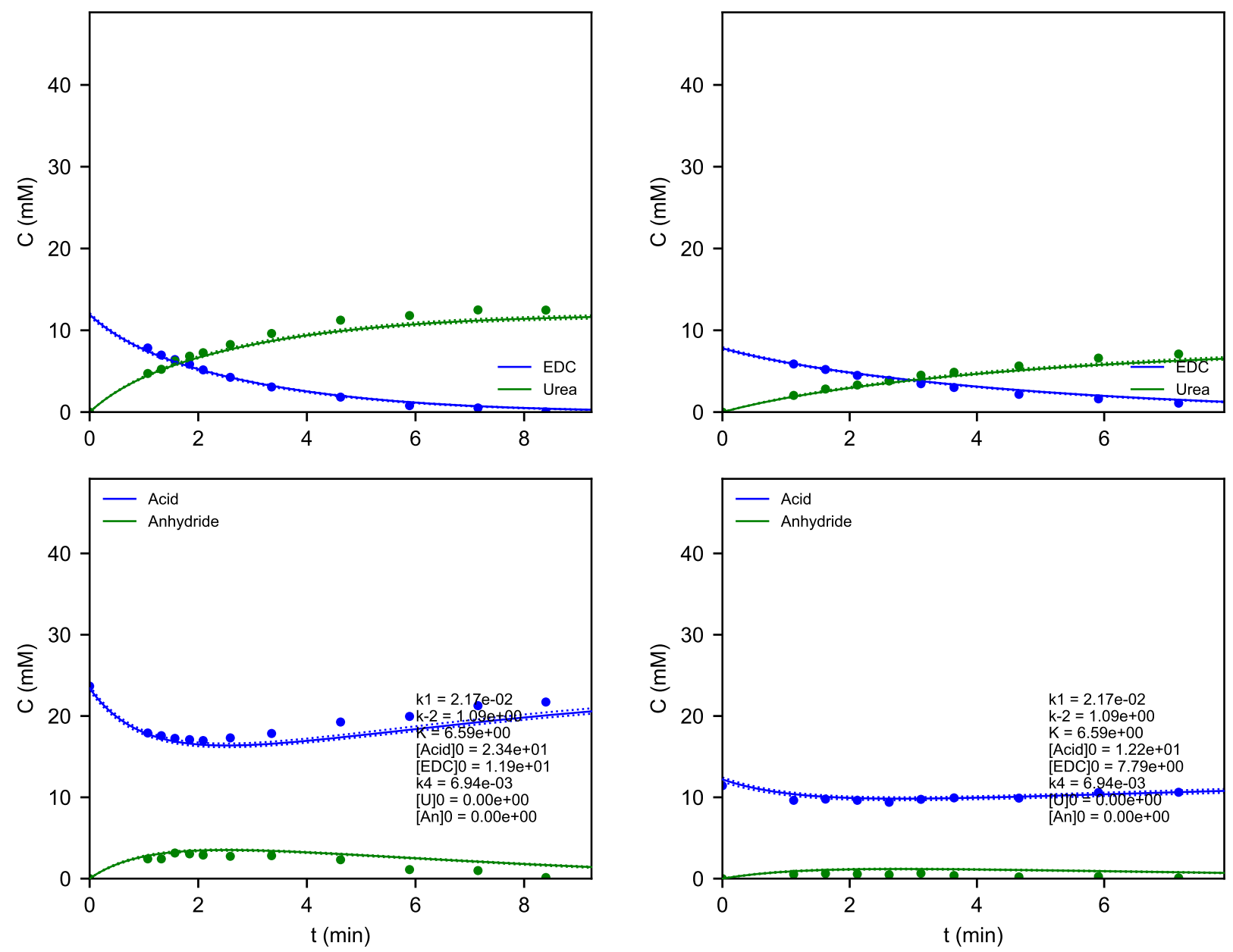

Figure S22. Continued.

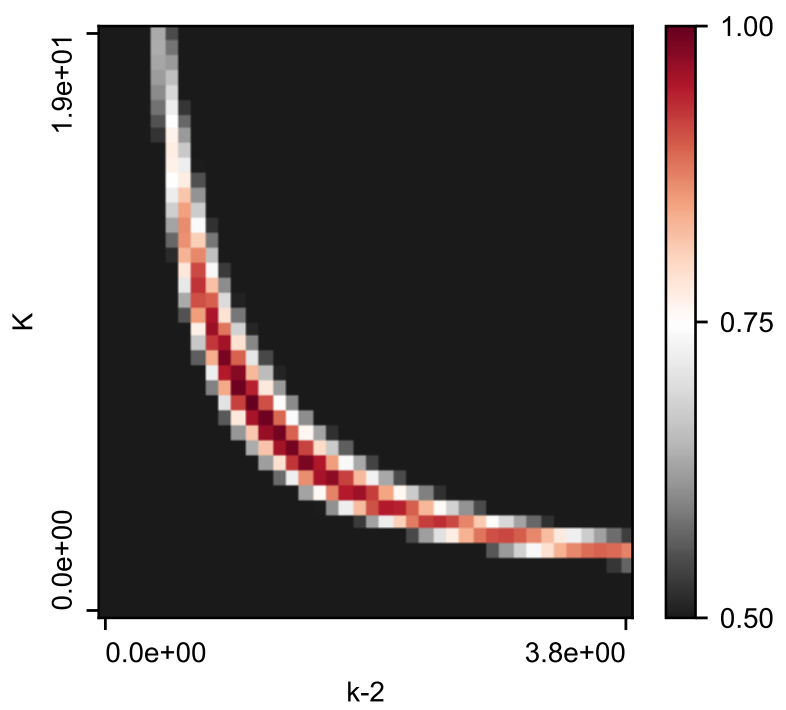

Figure S23. Heat map of the response of the regression error function to variation of $k_{-2}$ and $\alpha$ for the Ac3/An3 system ( $K$ in the figure is $\alpha$ ). The inverse error function is plotted, normalized against the value for the optimized system. 


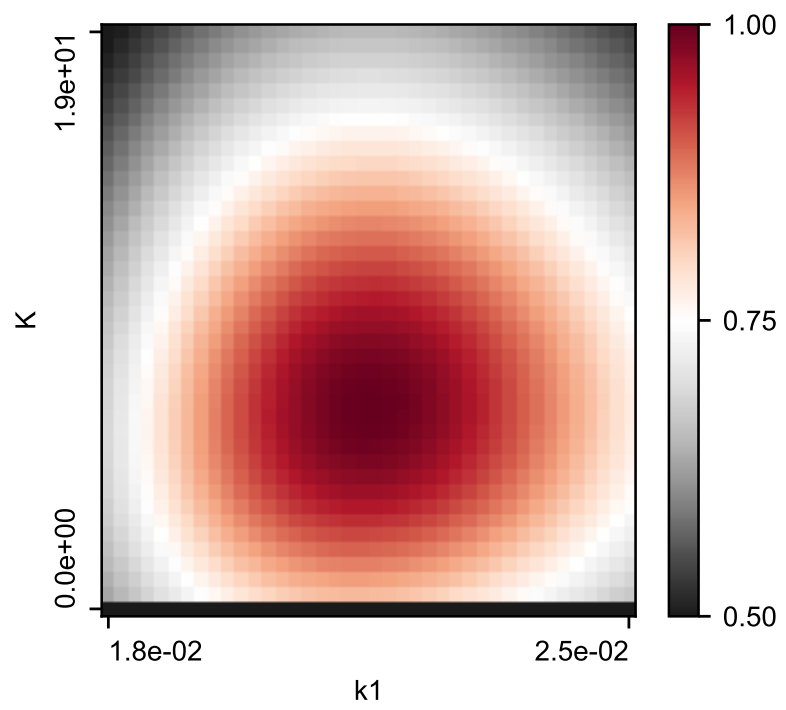

Figure S24. Heat map of the response of the regression error function to variation of $k_{1}$ and $\alpha$ for the Ac3/An3 system ( $K$ in the figure is $\alpha$ ). The inverse error function is plotted, normalized against the value for the optimized system.

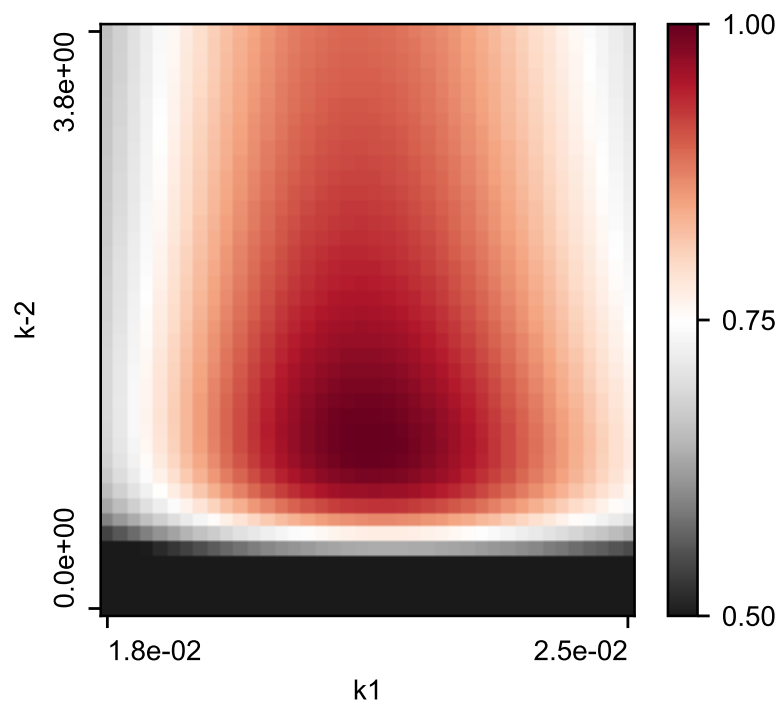

Figure S25. Heat map of the response of the regression error function to variation of $k_{1}$ and $k_{-2}$ for the Ac3/An3 system. The inverse error function is plotted, normalized against the value for the optimized system. 

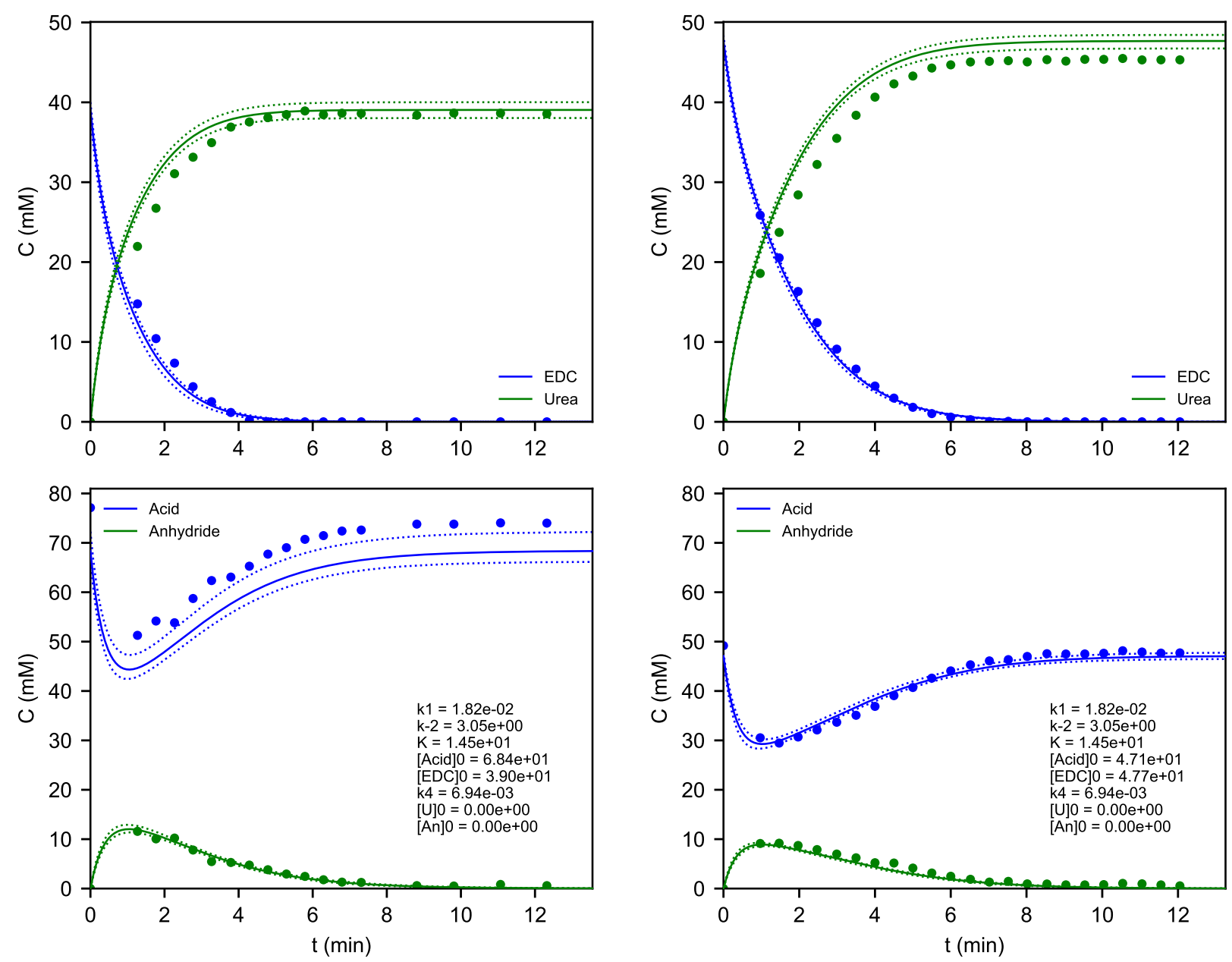

Figure S26. ${ }^{1} \mathrm{H}$ NMR spectroscopy monitoring of the treatment of Ac4 with EDC (0.5 M pyridine- $d_{5}$ buffer, pD 4.5, $\left.I=1.0 \mathrm{M}(\mathrm{NaCl}), \mathrm{D}_{2} \mathrm{O}, 298 \mathrm{~K}\right)$. The dashed lines represent $95 \%$ confidence intervals for the fits $(K$ in the figure is $\alpha$ ) (continues on next page). 

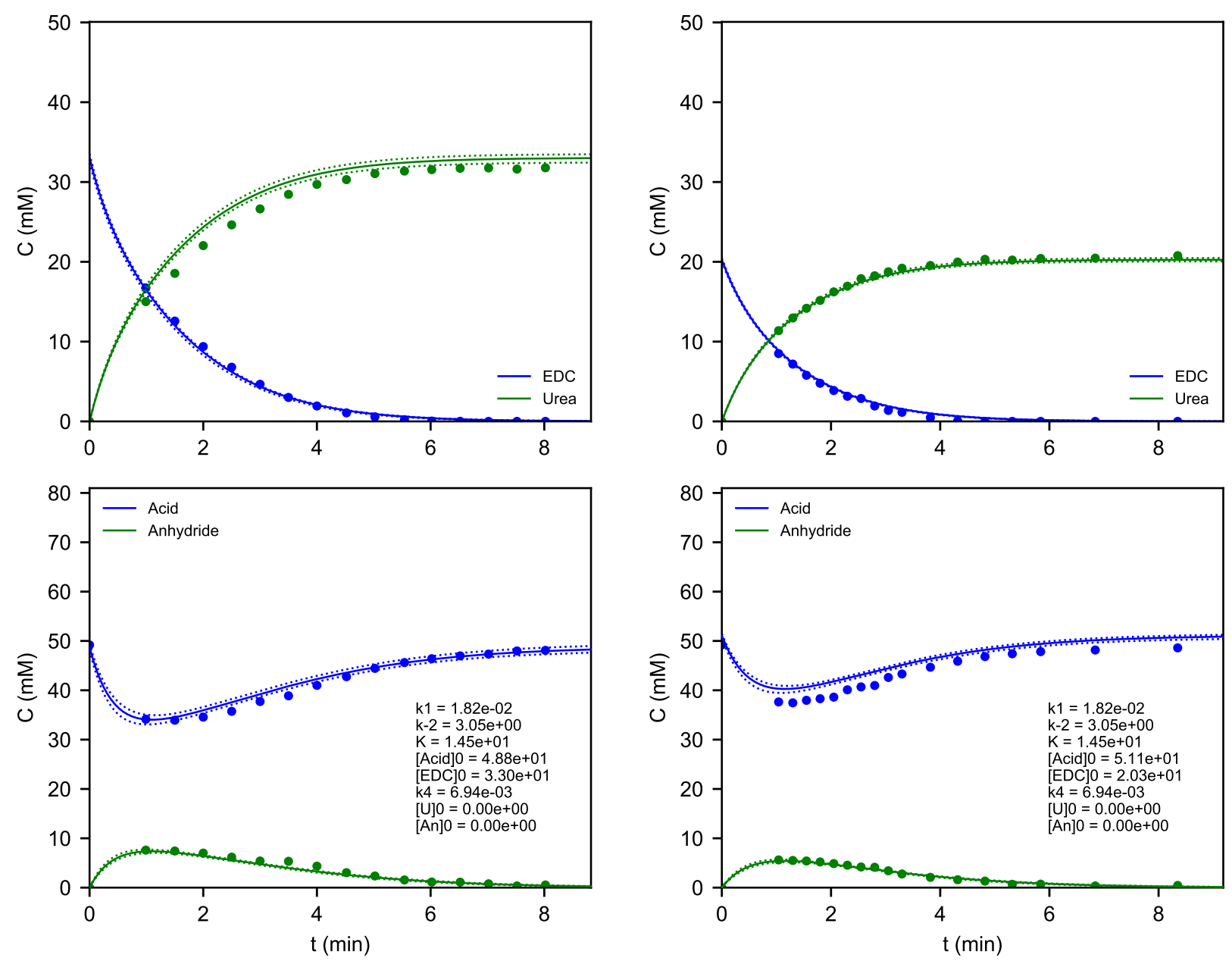

Figure S26. Continued. 

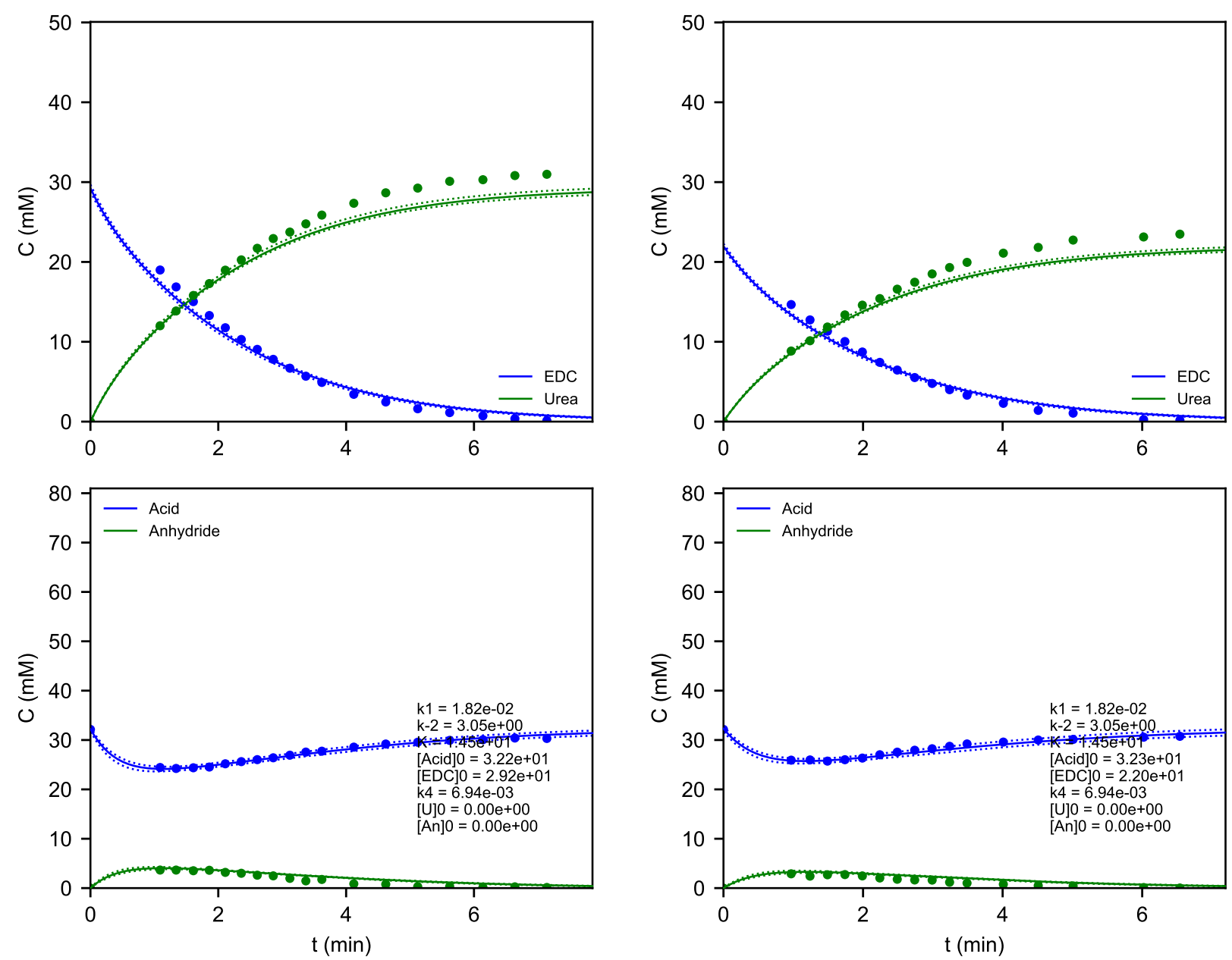

Figure S26. Continued. 

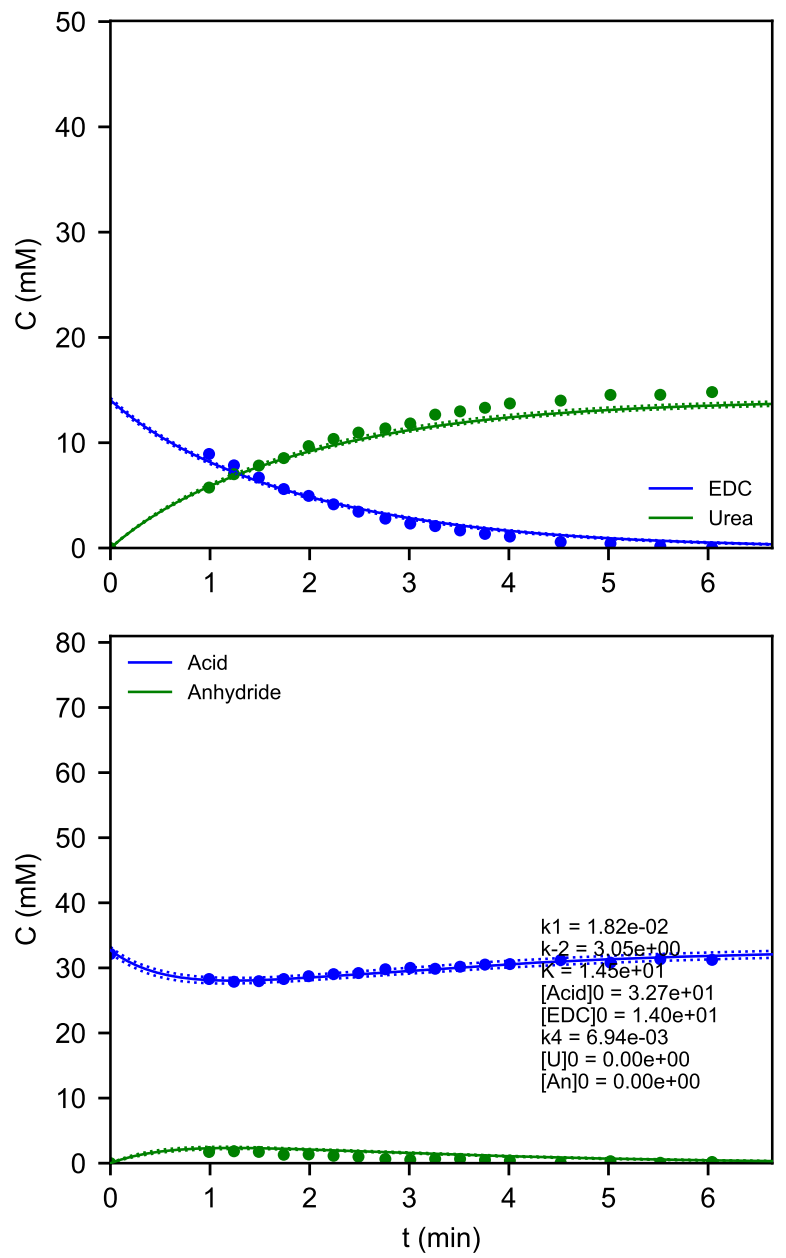

Figure S26. Continued.

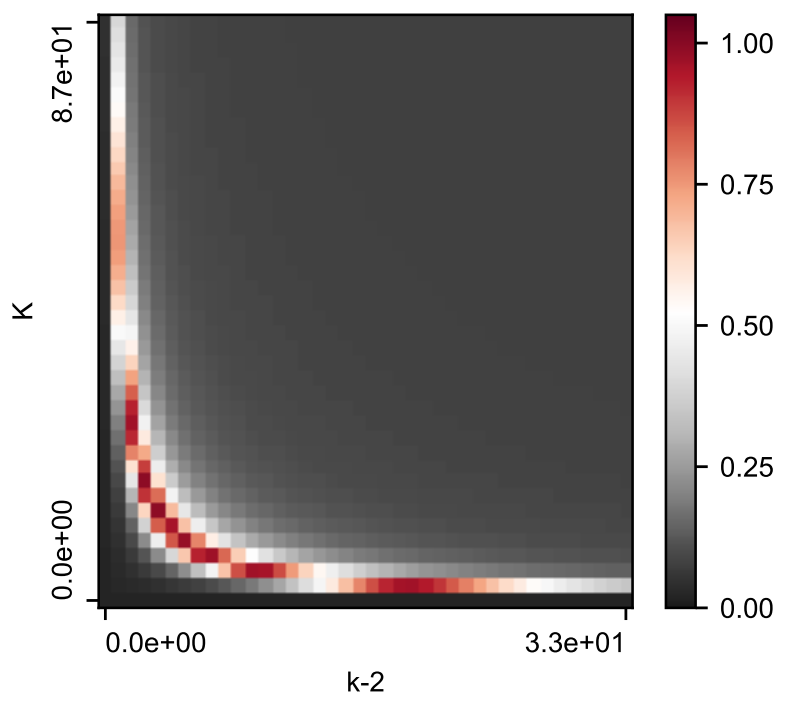

Figure S27. Heat map of the response of the regression error function to variation of $k_{-2}$ and $\alpha$ for the Ac4/An4 system ( $K$ in the figure is $\alpha$ ). The inverse error function is plotted, normalized against the value for the optimized system. 


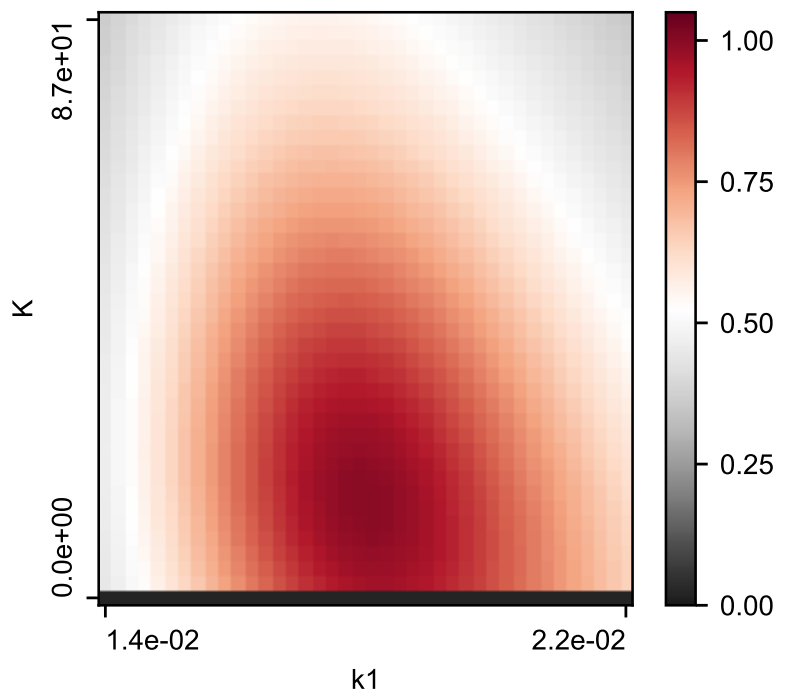

Figure S28. Heat map of the response of the regression error function to variation of $k_{1}$ and $\alpha$ for the Ac4/An4 system ( $K$ in the figure is $\alpha$ ). The inverse error function is plotted, normalized against the value for the optimized system.

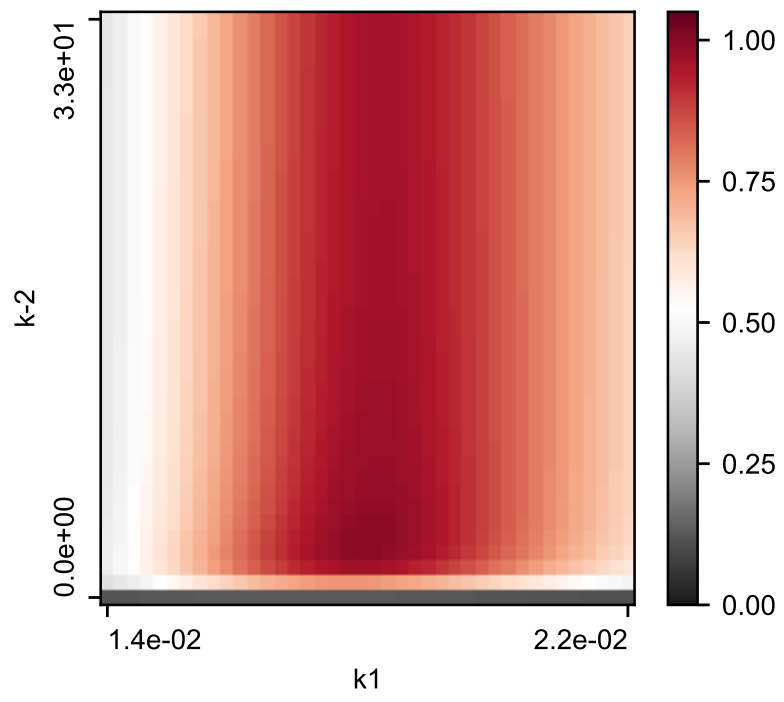

Figure S29. Heat map of the response of the regression error function to variation of $k_{1}$ and $k_{-2}$ for the Ac4/An4 system. The inverse error function is plotted, normalized against the value for the optimized system. 


\section{Background EDC hydrolysis}

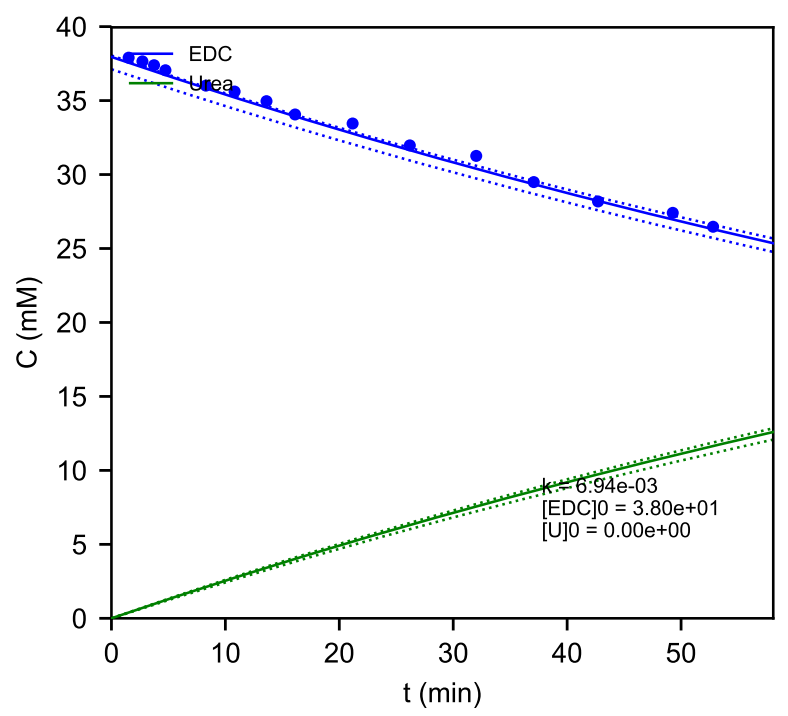

Figure S30. EDC hydrolysis kinetics. The solid line represents a fit to a simple first-order decay; the dashed lines are $95 \%$ confidence intervals for the regression. 

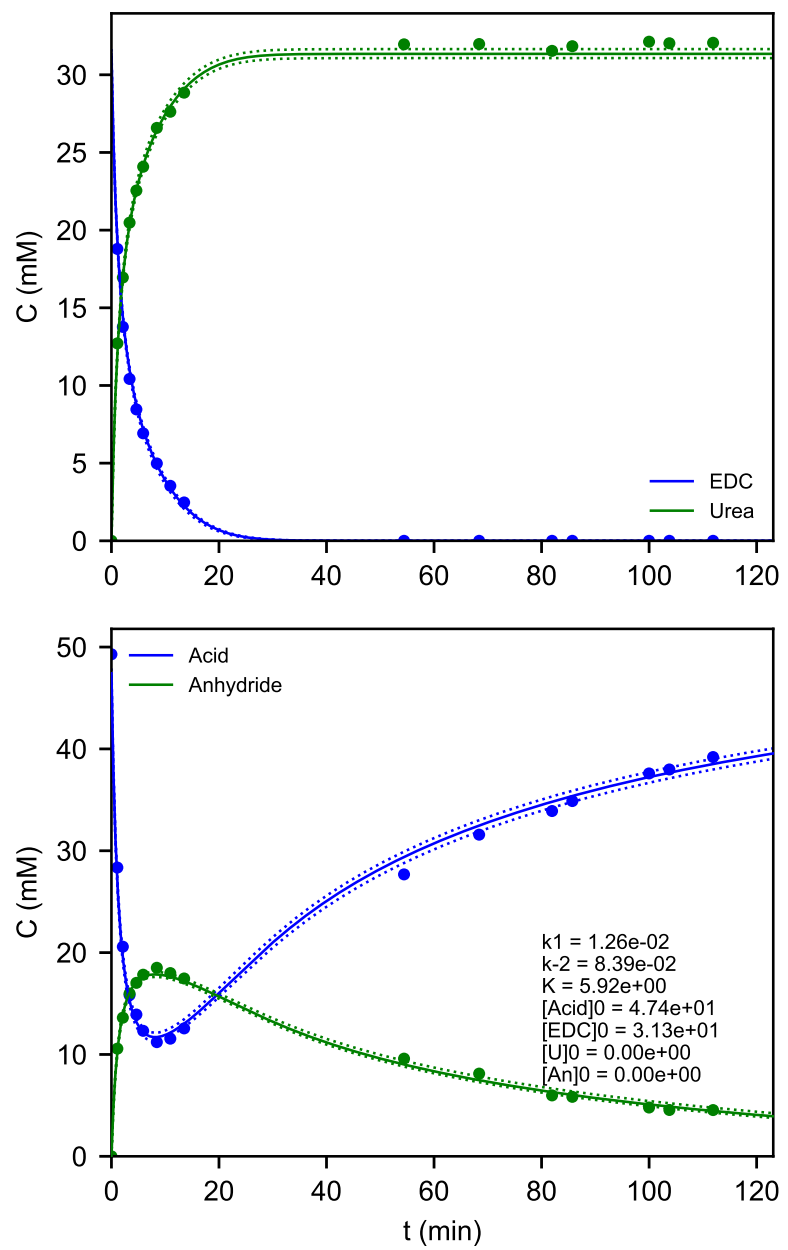

Figure S31. ${ }^{1} \mathrm{H}$ NMR spectroscopy monitoring of the treatment of Ac1 with EDC at $\mathrm{pD} 5.0$ (0.5 M pyridine$d_{5}$ buffer, $\left.I=1.0 \mathrm{M}(\mathrm{NaCl}), \mathrm{D}_{2} \mathrm{O}, 298 \mathrm{~K}\right)$. The dashed lines represent $95 \%$ confidence intervals for the fits $(K$ in the figure is $\alpha$ ). 

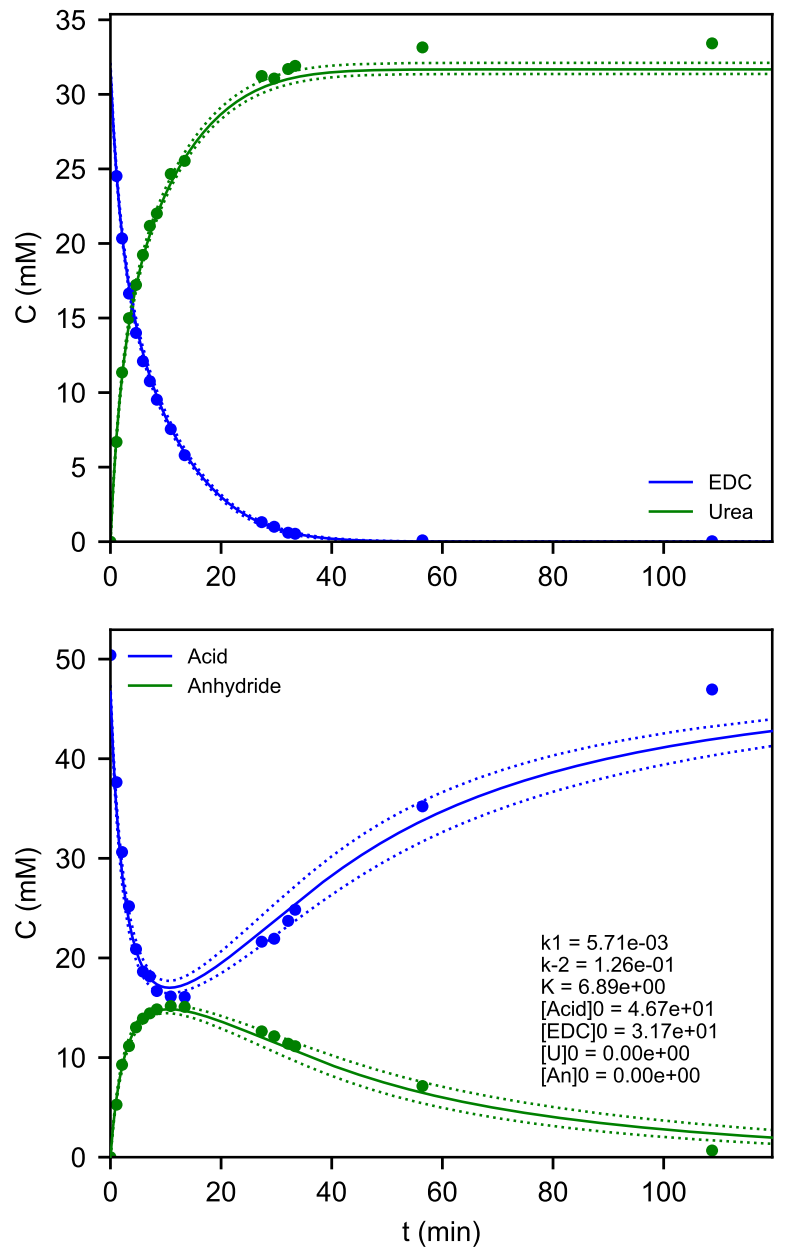

Figure S32. ${ }^{1} \mathrm{H}$ NMR spectroscopy monitoring of the treatment of Ac1 with EDC at $\mathrm{pD} 5.5$ (0.5 M pyridine$d_{5}$ buffer, $\left.I=1.0 \mathrm{M}(\mathrm{NaCl}), \mathrm{D}_{2} \mathrm{O}, 298 \mathrm{~K}\right)$. The dashed lines represent $95 \%$ confidence intervals for the fits $(K$ in the figure is $\alpha$ ). 

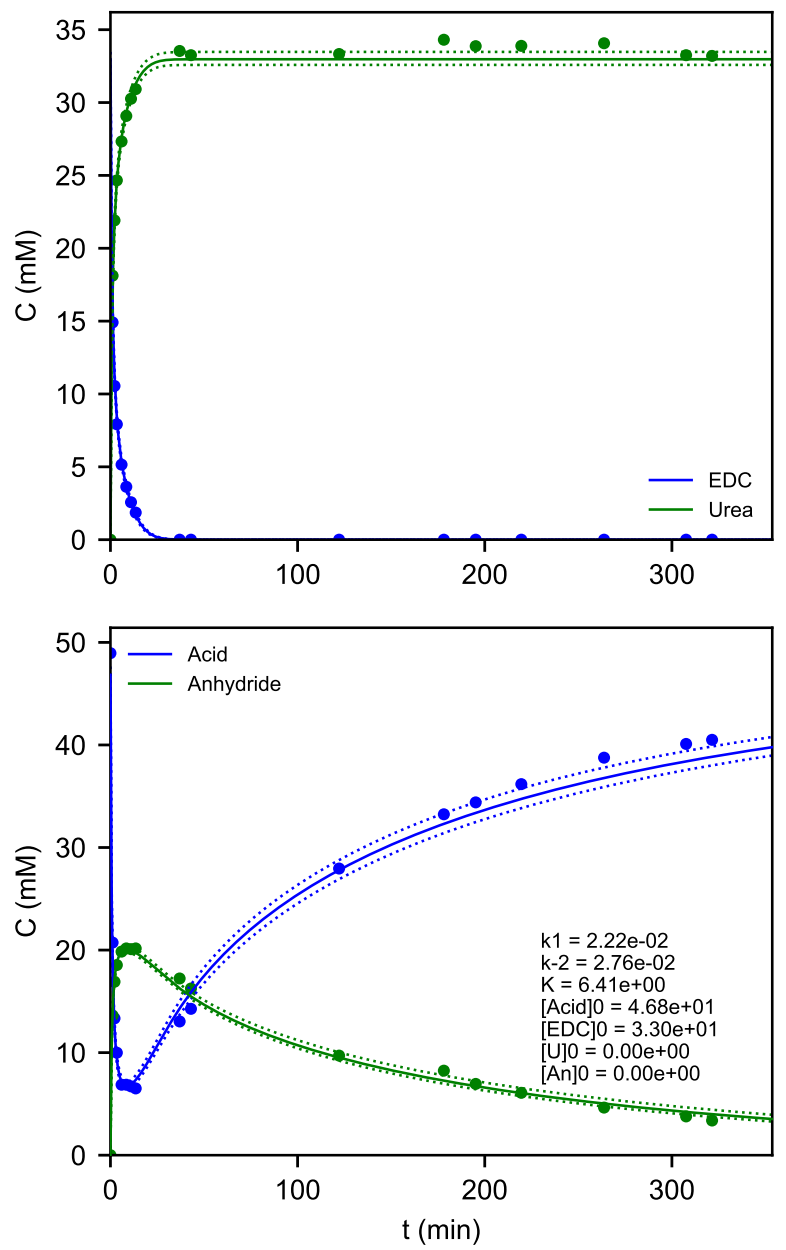

Figure S33. ${ }^{1} \mathrm{H}$ NMR spectroscopy monitoring of the treatment of Ac1 with EDC at $0.4 \mathrm{M}$ pyridine- $d_{5}$ buffer $\left(\mathrm{pD} 4.5, I=1.0 \mathrm{M}(\mathrm{NaCl}), \mathrm{D}_{2} \mathrm{O}, 298 \mathrm{~K}\right)$. The dashed lines represent $95 \%$ confidence intervals for the fits $(K$ in the figure is $\alpha$ ). 

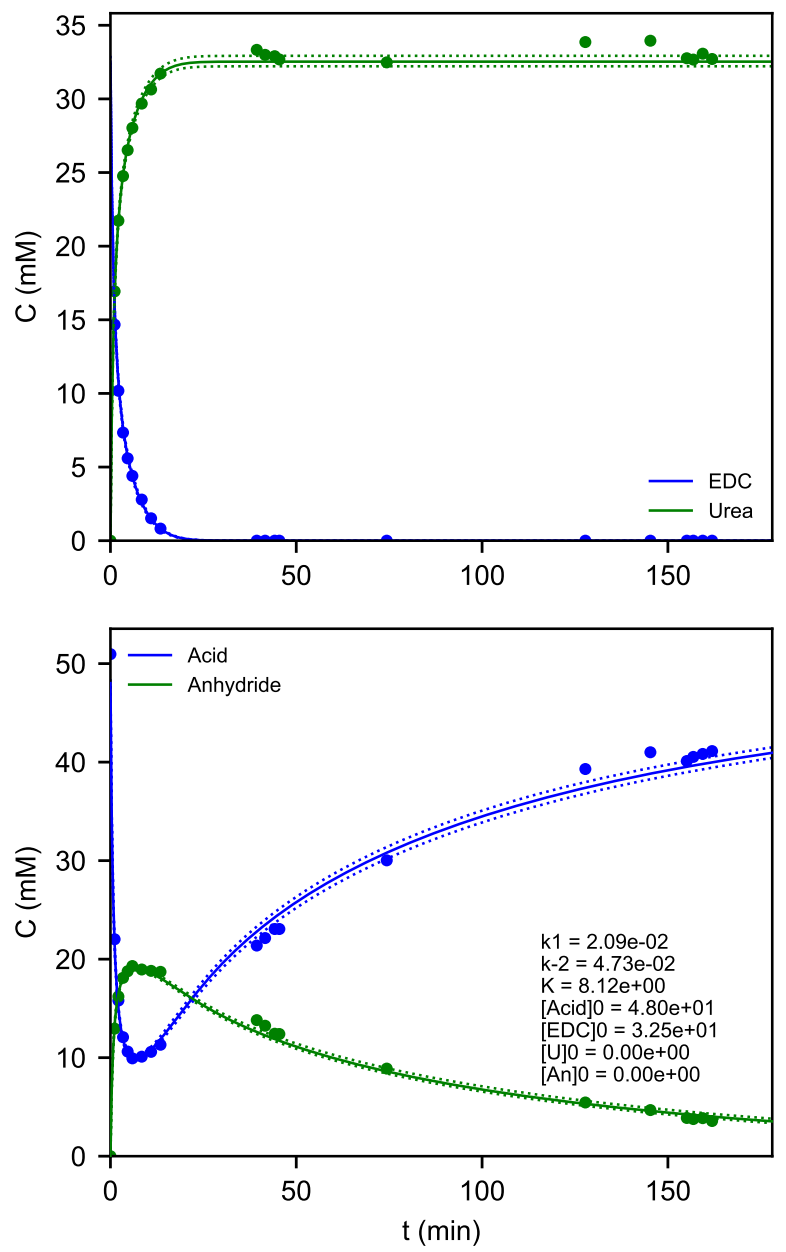

Figure S34. ${ }^{1} \mathrm{H}$ NMR spectroscopy monitoring of the treatment of Ac1 with EDC at $0.6 \mathrm{M}$ pyridine- $d_{5}$ buffer $\left(\mathrm{pD} 4.5, I=1.0 \mathrm{M}(\mathrm{NaCl}), \mathrm{D}_{2} \mathrm{O}, 298 \mathrm{~K}\right)$. The dashed lines represent $95 \%$ confidence intervals for the fits $(K$ in the figure is $\alpha$ ). 

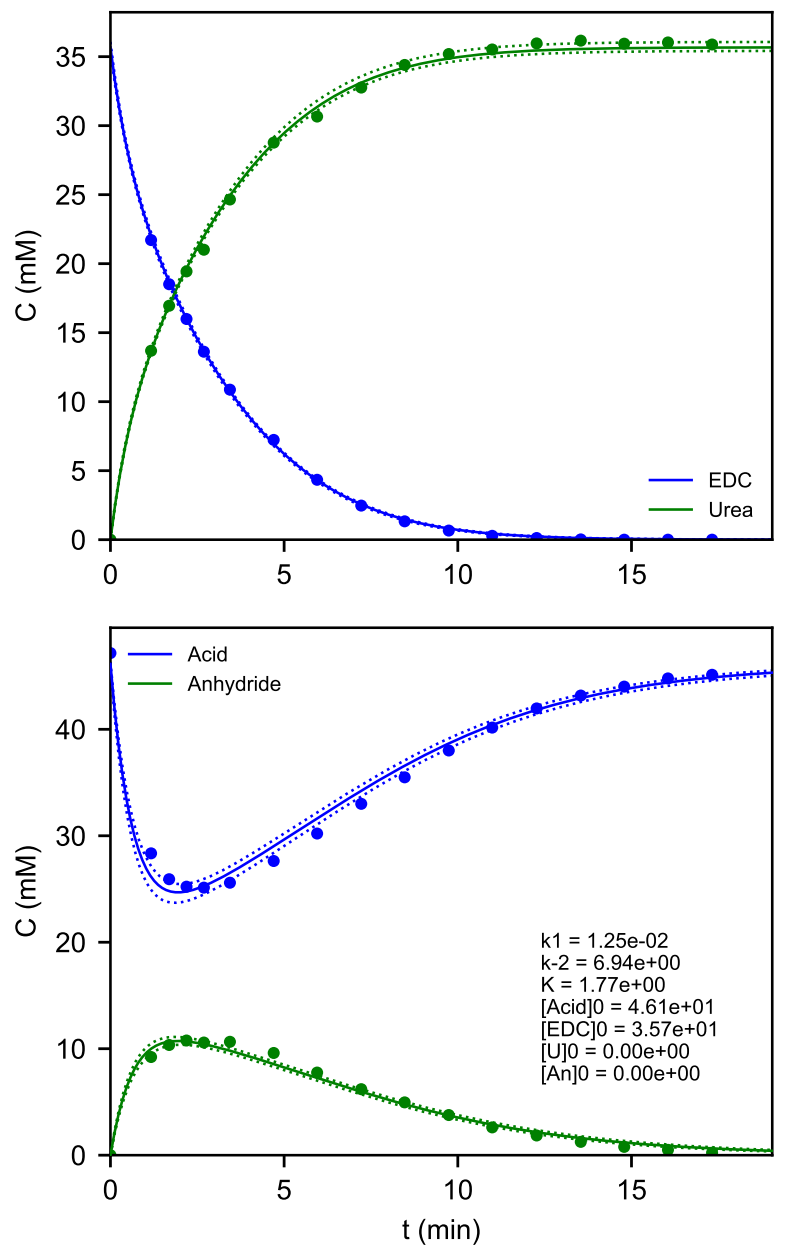

Figure S35. ${ }^{1} \mathrm{H}$ NMR spectroscopy monitoring of the treatment of Ac2 with EDC at pD 5.0 (0.5 M pyridine$d_{5}$ buffer, $\left.I=1.0 \mathrm{M}(\mathrm{NaCl}), \mathrm{D}_{2} \mathrm{O}, 298 \mathrm{~K}\right)$. The dashed lines represent $95 \%$ confidence intervals for the fits $(K$ in the figure is $\alpha$ ). 

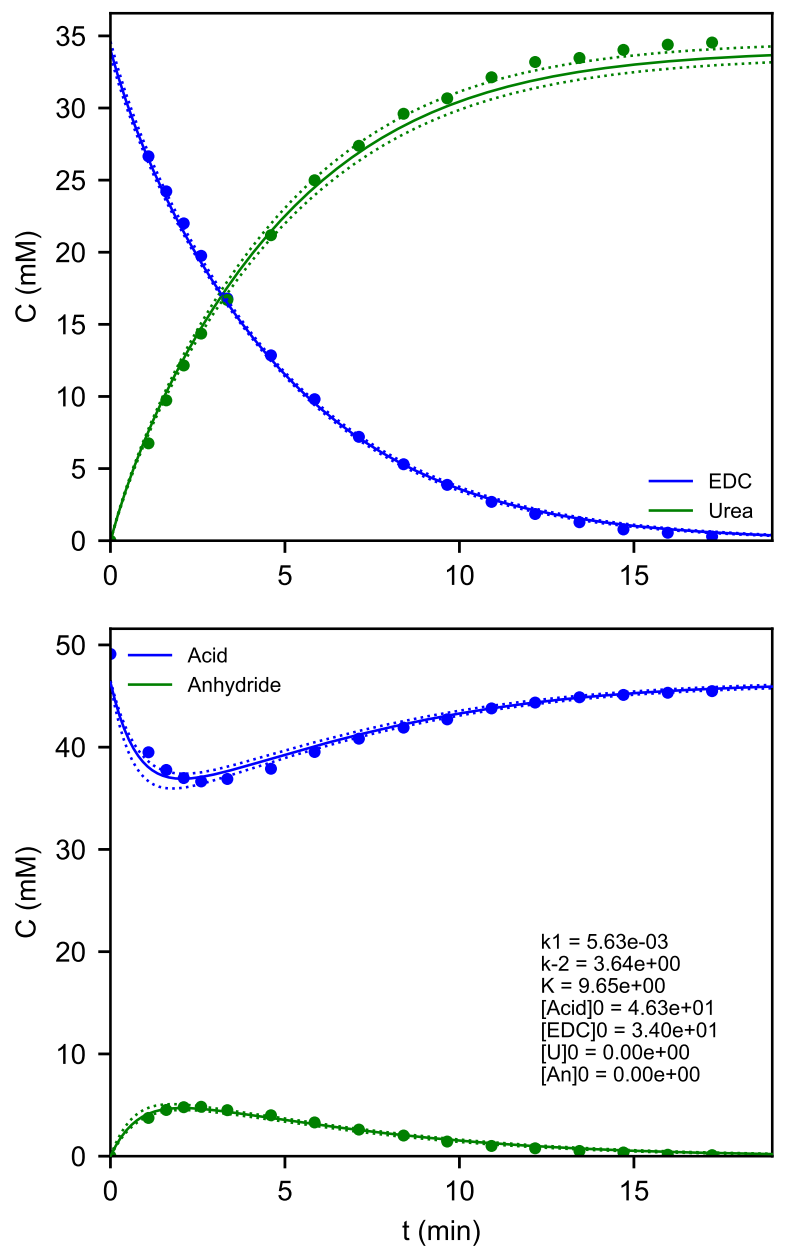

Figure S36. ${ }^{1} \mathrm{H}$ NMR spectroscopy monitoring of the treatment of Ac2 with EDC at $\mathrm{pD} 5.5$ (0.5 M pyridine$d_{5}$ buffer, $\left.I=1.0 \mathrm{M}(\mathrm{NaCl}), \mathrm{D}_{2} \mathrm{O}, 298 \mathrm{~K}\right)$. The dashed lines represent $95 \%$ confidence intervals for the fits $(K$ in the figure is $\alpha$ ). 

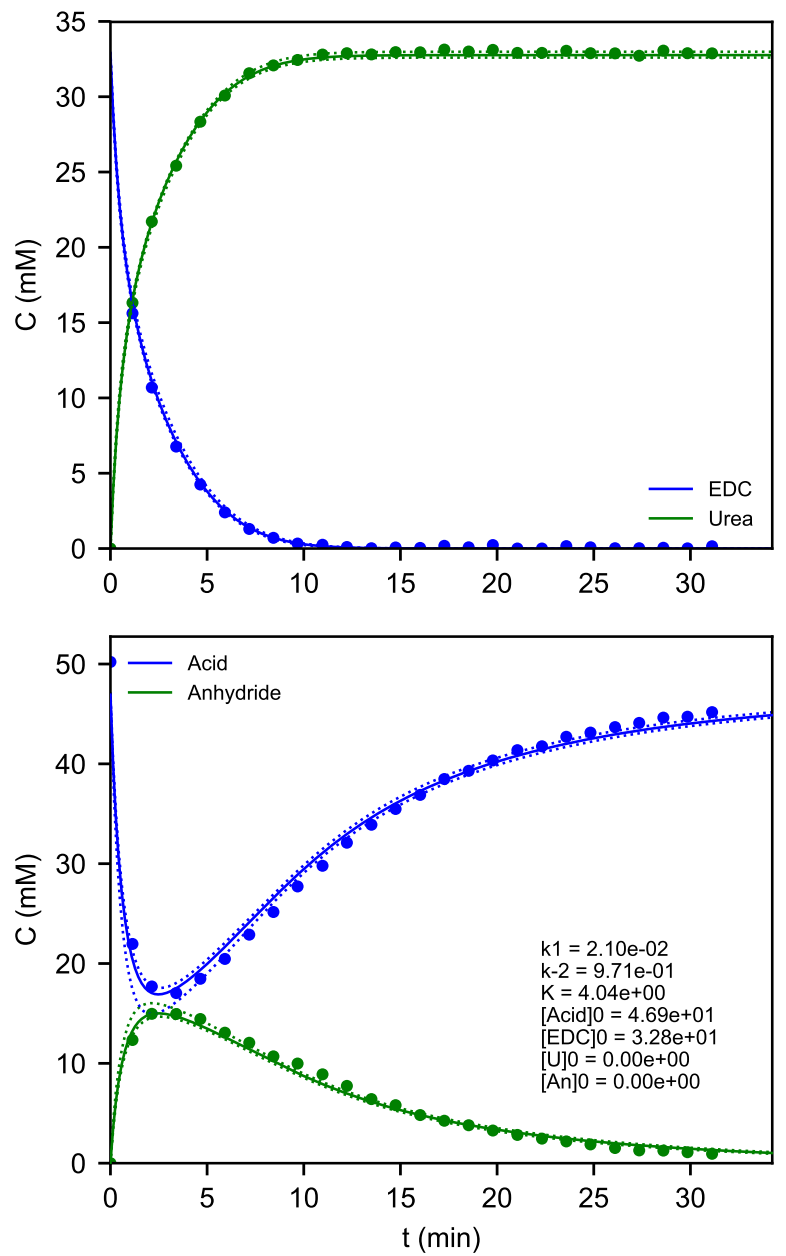

Figure S37. ${ }^{1} \mathrm{H}$ NMR spectroscopy monitoring of the treatment of Ac2 with EDC at $0.4 \mathrm{M}$ pyridine- $d_{5}$ buffer $\left(\mathrm{pD} 4.5, I=1.0 \mathrm{M}(\mathrm{NaCl}), \mathrm{D}_{2} \mathrm{O}, 298 \mathrm{~K}\right)$. The dashed lines represent $95 \%$ confidence intervals for the fits $(K$ in the figure is $\alpha$ ). 

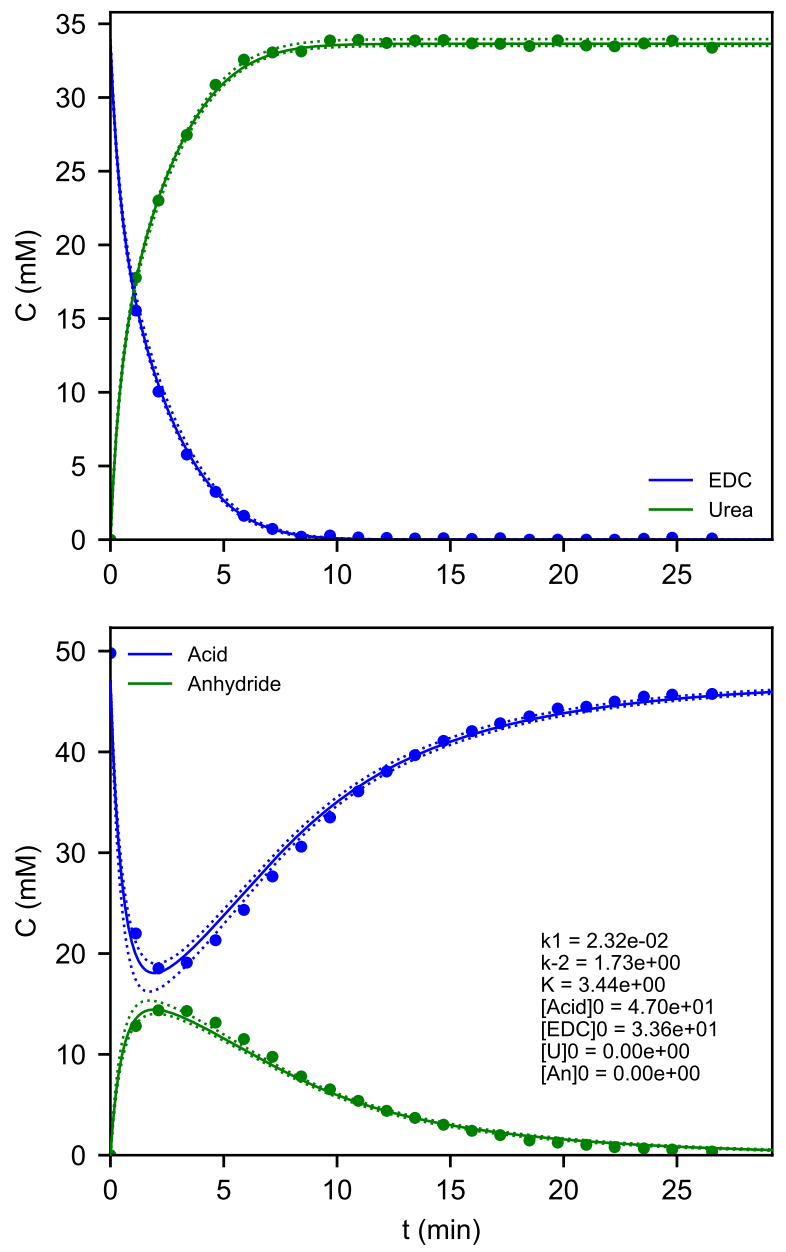

Figure S38. ${ }^{1} \mathrm{H}$ NMR spectroscopy monitoring of the treatment of Ac2 with EDC at $0.6 \mathrm{M}$ pyridine- $d_{5}$ buffer $\left(\mathrm{pD} 4.5, I=1.0 \mathrm{M}(\mathrm{NaCl}), \mathrm{D}_{2} \mathrm{O}, 298 \mathrm{~K}\right)$. The dashed lines represent $95 \%$ confidence intervals for the fits $(K$ in the figure is $\alpha$ ). 

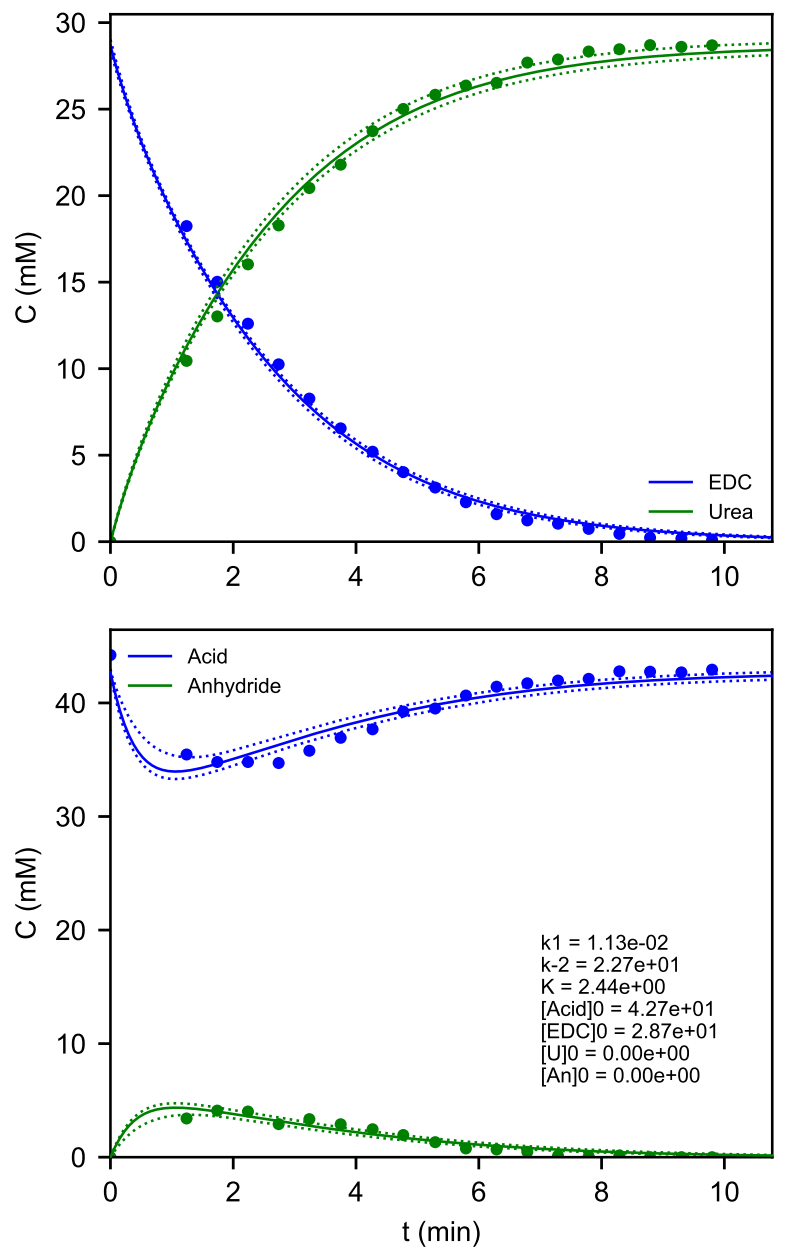

Figure S39. ${ }^{1} \mathrm{H}$ NMR spectroscopy monitoring of the treatment of Ac3 with EDC at pD 5.0 (0.5 M pyridine$d_{5}$ buffer, $\left.I=1.0 \mathrm{M}(\mathrm{NaCl}), \mathrm{D}_{2} \mathrm{O}, 298 \mathrm{~K}\right)$. The dashed lines represent $95 \%$ confidence intervals for the fits $(K$ in the figure is $\alpha$ ). 

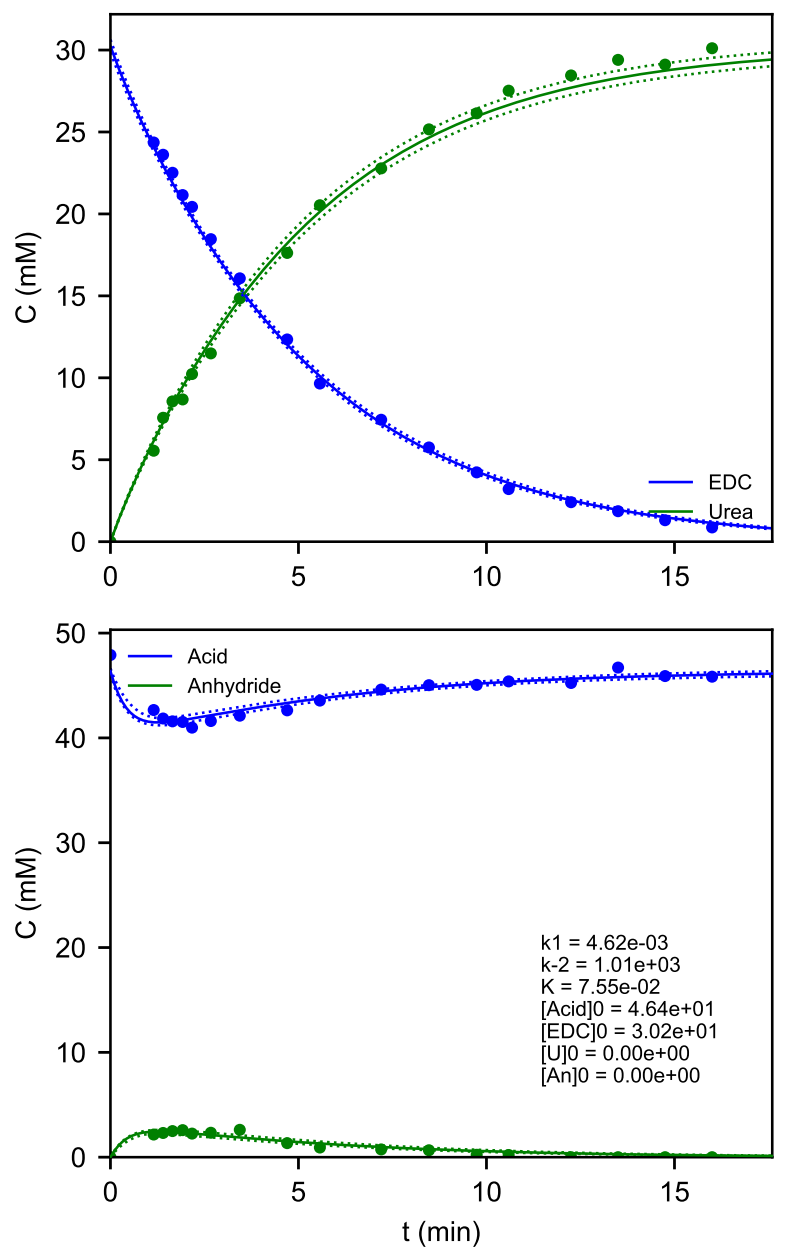

Figure S40. ${ }^{1} \mathrm{H}$ NMR spectroscopy monitoring of the treatment of Ac3 with EDC at $\mathrm{pD} 5.5$ (0.5 M pyridine$d_{5}$ buffer, $\left.I=1.0 \mathrm{M}(\mathrm{NaCl}), \mathrm{D}_{2} \mathrm{O}, 298 \mathrm{~K}\right)$. The dashed lines represent $95 \%$ confidence intervals for the fits $(K$ in the figure is $\alpha$ ). 

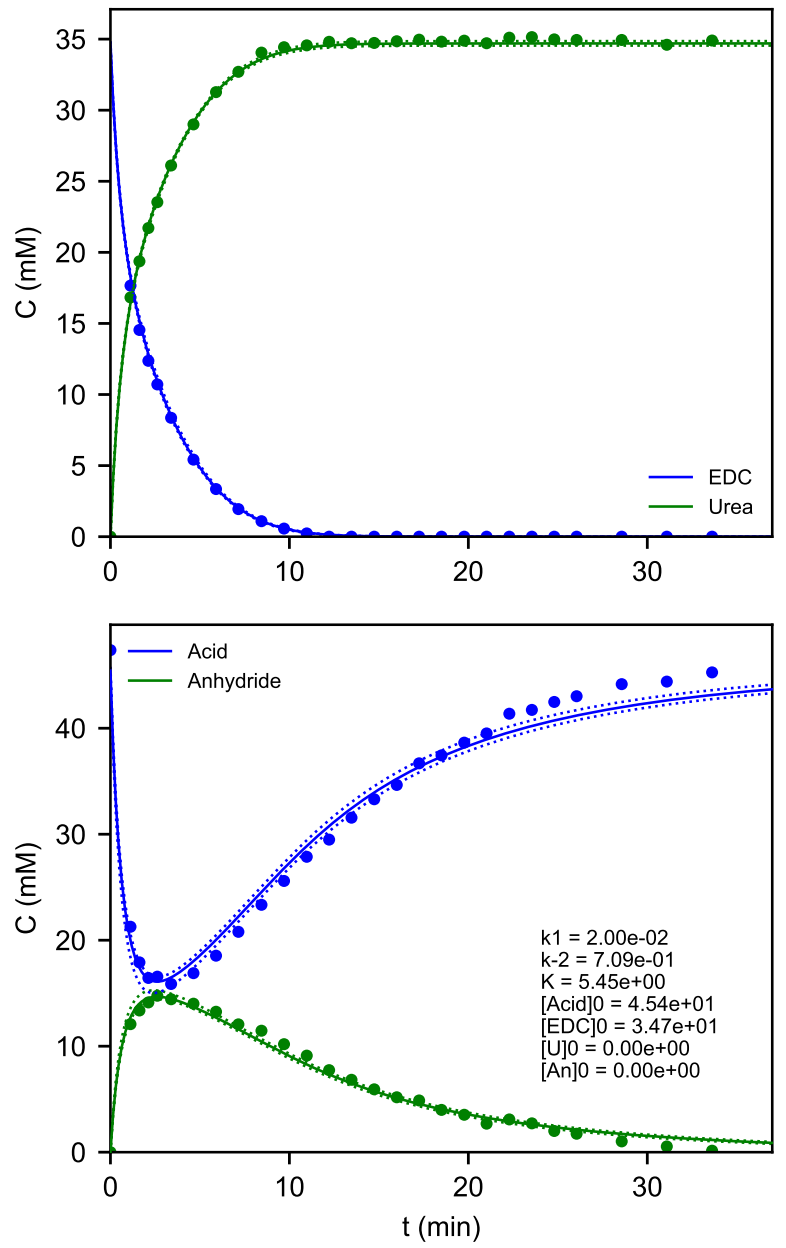

Figure S41. ${ }^{1} \mathrm{H}$ NMR spectroscopy monitoring of the treatment of Ac3 with EDC at $0.4 \mathrm{M}$ pyridine- $d_{5}$ buffer $\left(\mathrm{pD} 4.5, I=1.0 \mathrm{M}(\mathrm{NaCl}), \mathrm{D}_{2} \mathrm{O}, 298 \mathrm{~K}\right)$. The dashed lines represent $95 \%$ confidence intervals for the fits $(K$ in the figure is $\alpha$ ). 

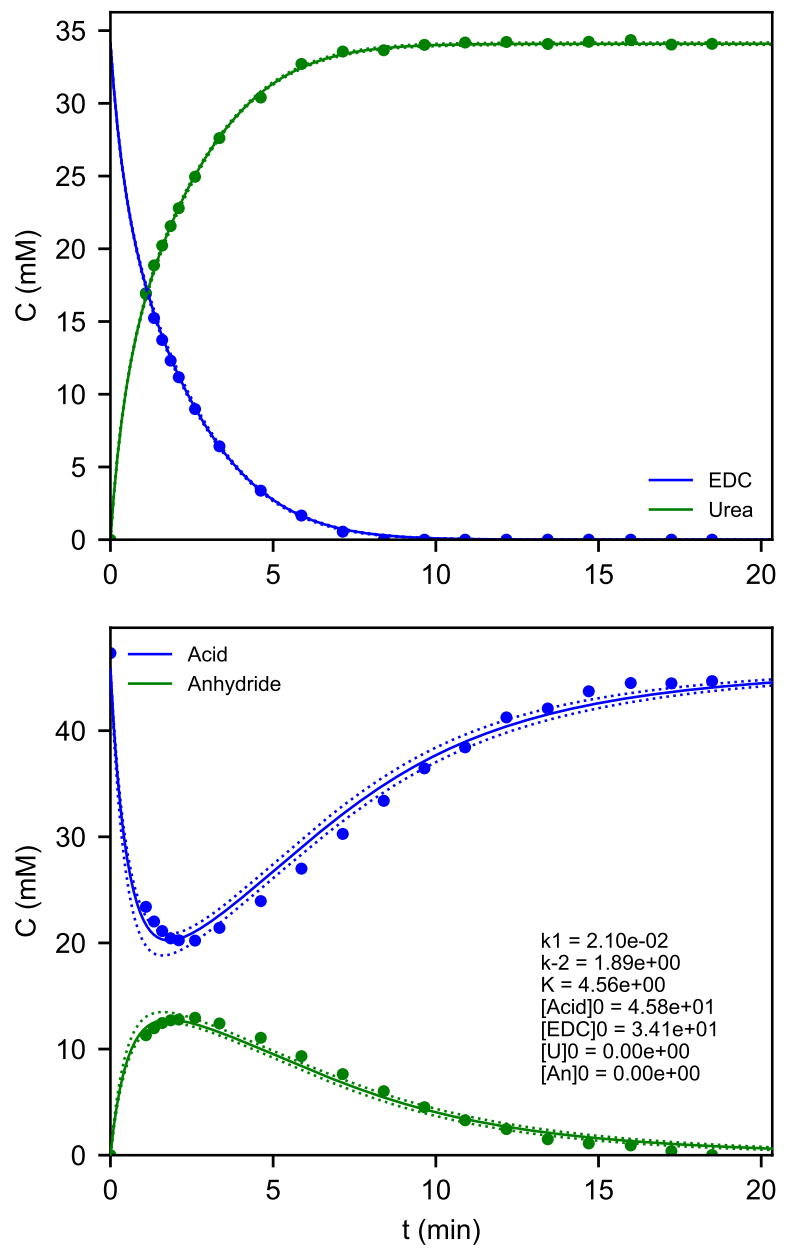

Figure S42. ${ }^{1} \mathrm{H}$ NMR spectroscopy monitoring of the treatment of Ac3 with EDC at $0.6 \mathrm{M}$ pyridine- $d_{5}$ buffer $\left(\mathrm{pD} 4.5, I=1.0 \mathrm{M}(\mathrm{NaCl}), \mathrm{D}_{2} \mathrm{O}, 298 \mathrm{~K}\right)$. The dashed lines represent $95 \%$ confidence intervals for the fits $(K$ in the figure is $\alpha$ ). 

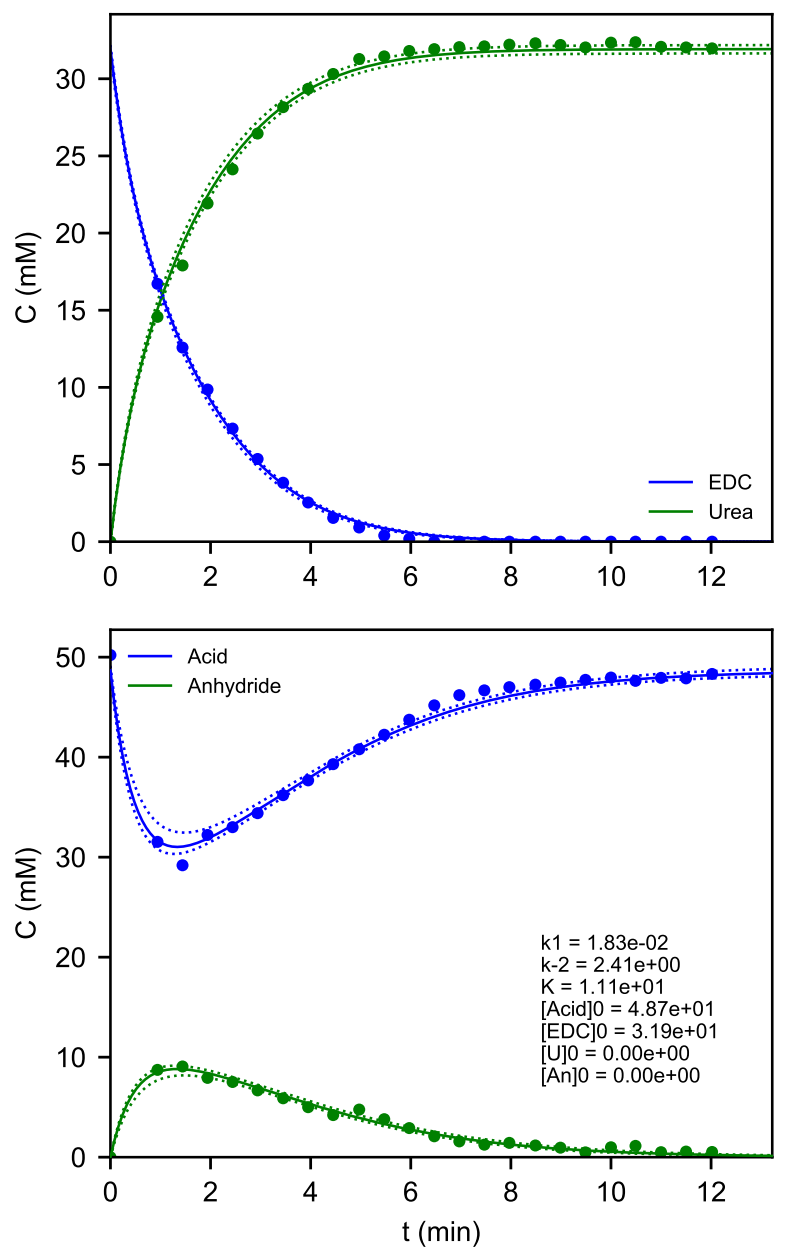

Figure S43. ${ }^{1} \mathrm{H}$ NMR spectroscopy monitoring of the treatment of Ac4 with EDC at $0.4 \mathrm{M}$ pyridine- $d_{5}$ buffer $\left(\mathrm{pD} 4.5, I=1.0 \mathrm{M}(\mathrm{NaCl}), \mathrm{D}_{2} \mathrm{O}, 298 \mathrm{~K}\right)$. The dashed lines represent $95 \%$ confidence intervals for the fits $(K$ in the figure is $\alpha$ ). 

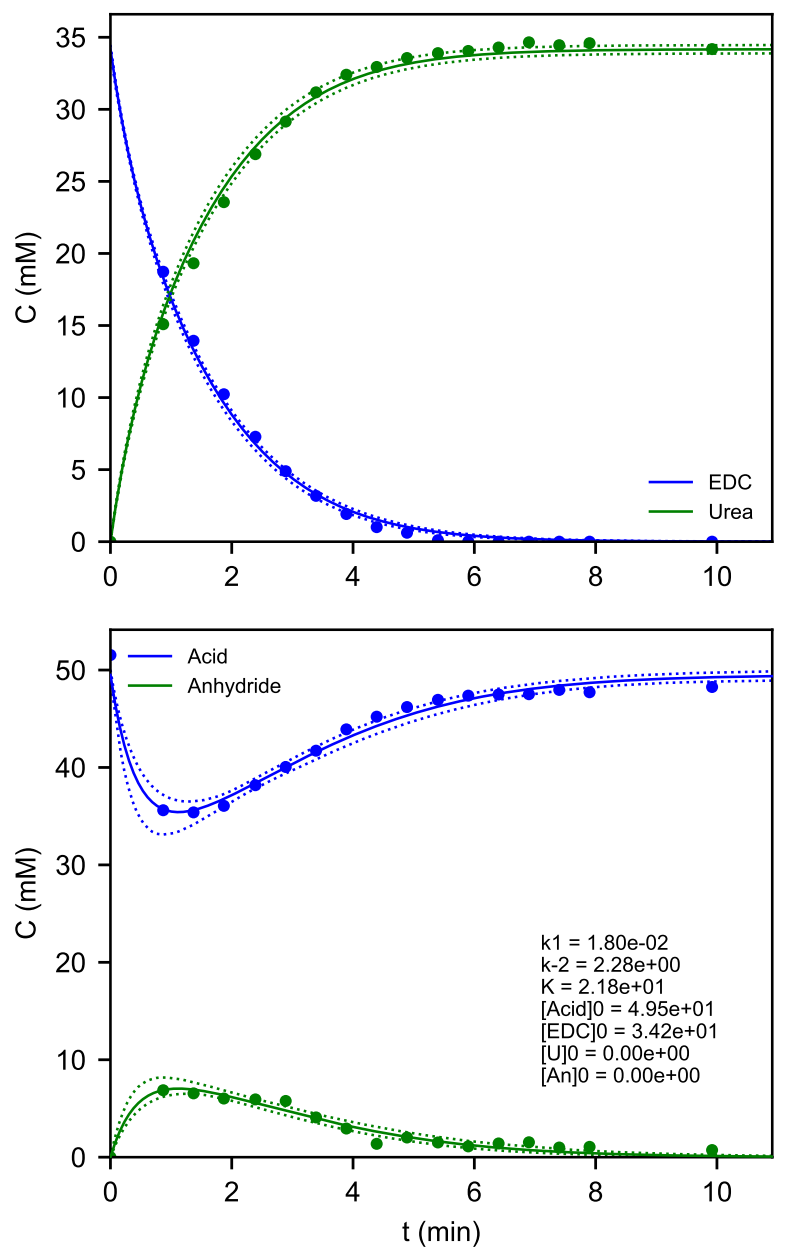

Figure S44. ${ }^{1} \mathrm{H}$ NMR spectroscopy monitoring of the treatment of Ac4 with EDC at $0.6 \mathrm{M}$ pyridine- $d_{5}$ buffer $\left(\mathrm{pD} 4.5, I=1.0 \mathrm{M}(\mathrm{NaCl}), \mathrm{D}_{2} \mathrm{O}, 298 \mathrm{~K}\right)$. The dashed lines represent $95 \%$ confidence intervals for the fits $(K$ in the figure is $\alpha$ ).

\section{Kinetic simulations}

Kinetic simulations were done using the model_kinetics program of the kinmodel package discussed above. Briefly, this program uses the same kinetic models as fit_kinetics to simulate kinetic runs for a set of parameters and starting concentrations. It calculates the same properties that are calculated during the kinetic fits. It accepts ranges of parameters and thus can be used to generate data for how various properties (e.g., yield) vary with starting conditions or parameter values.

Help for this program can be obtained with model_kinetics -h (the list of models is given in fit_kinetics -h). A typical simulation would be run with

model_kinetics model_name time param_values -f filename -n sim_num

where model_name is replaced with the model name; time is replaced with the simulation time; param_values is a list of the parameter values and concentrations (the order is specified in the model descriptions; variation of a parameter from $A$ to $B$ is given as A. . B); - $f$ filename provides the output filename; and $-n$ sim_num gives the total number of simulations to perform (i.e., for a set of parameter ranges). The program outputs a text file summarizing the simulations and a pdf concentration vs time plot. Other options can be used to simplify the output if a large number of simulations is going to be performed (e.g., omit the pdf figures). 
NMR spectra of synthesized compounds

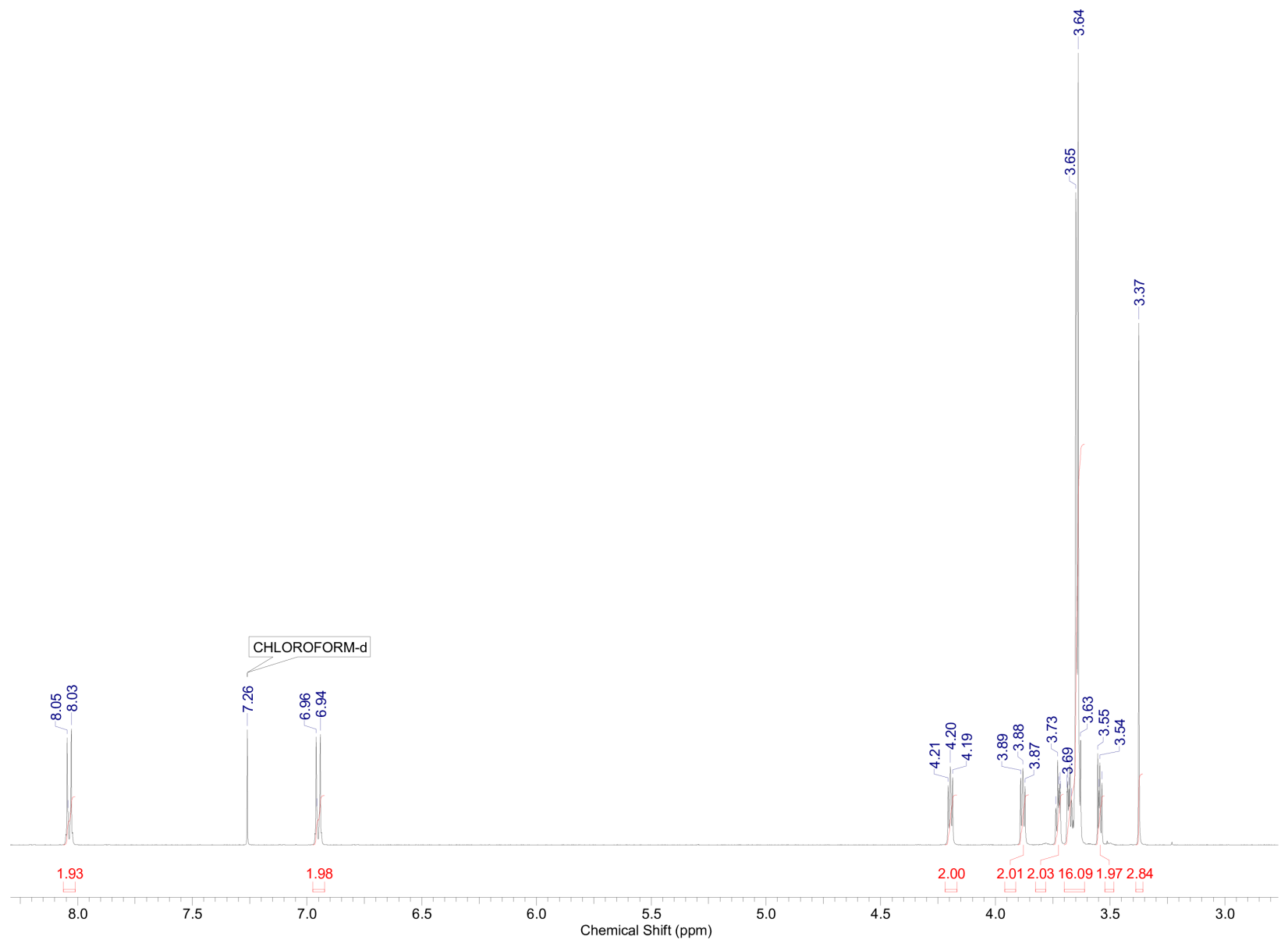

Figure S45. ${ }^{1} \mathrm{H}$ NMR spectrum $\left(500 \mathrm{MHz}, \mathrm{CDCl}_{3}\right)$ of Ac1. 


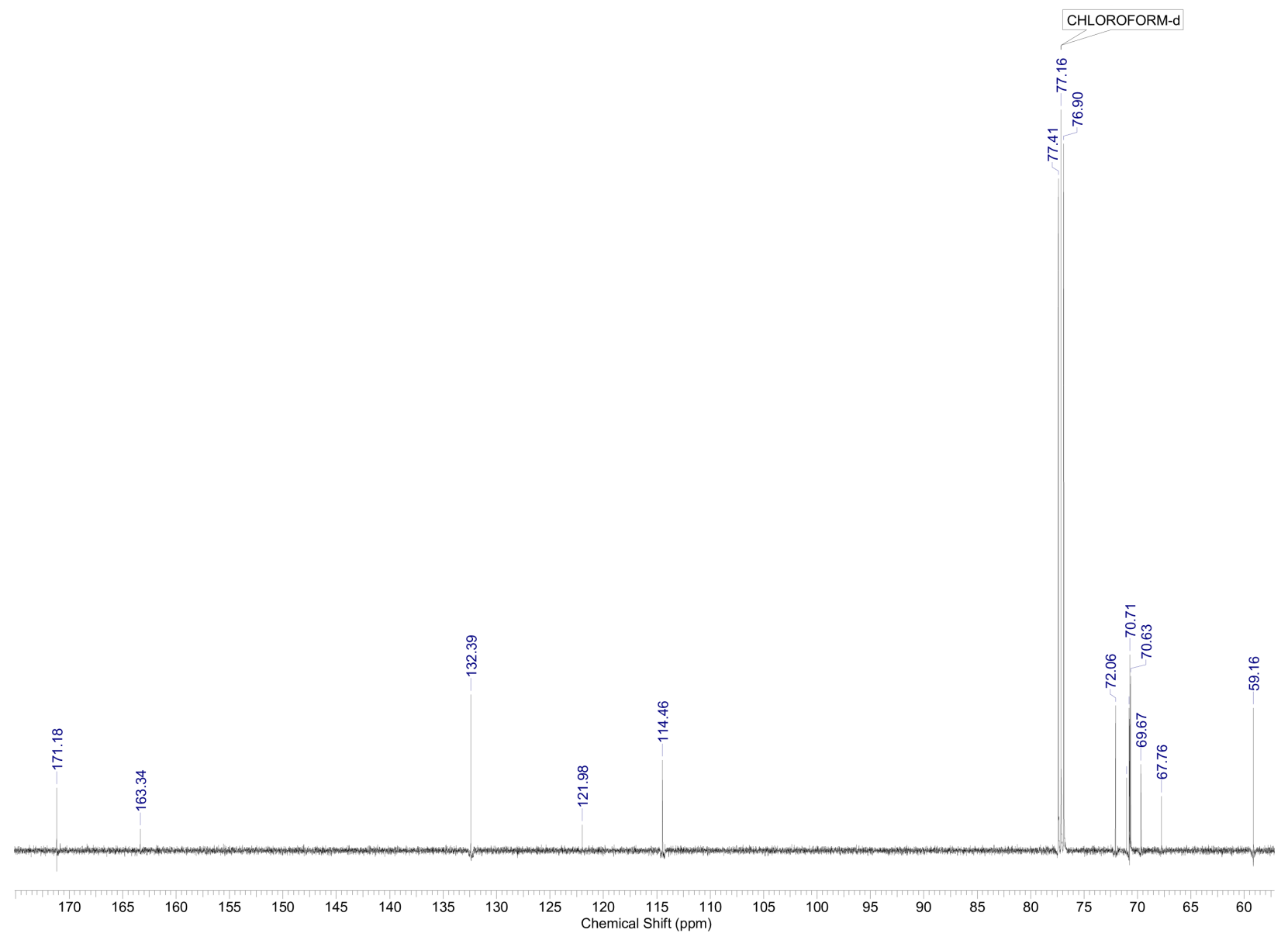

Figure S46. ${ }^{13} \mathrm{C}\left\{{ }^{1} \mathrm{H}\right\}$ NMR spectrum $\left(125 \mathrm{MHz}, \mathrm{CDCl}_{3}\right)$ of Ac1. 


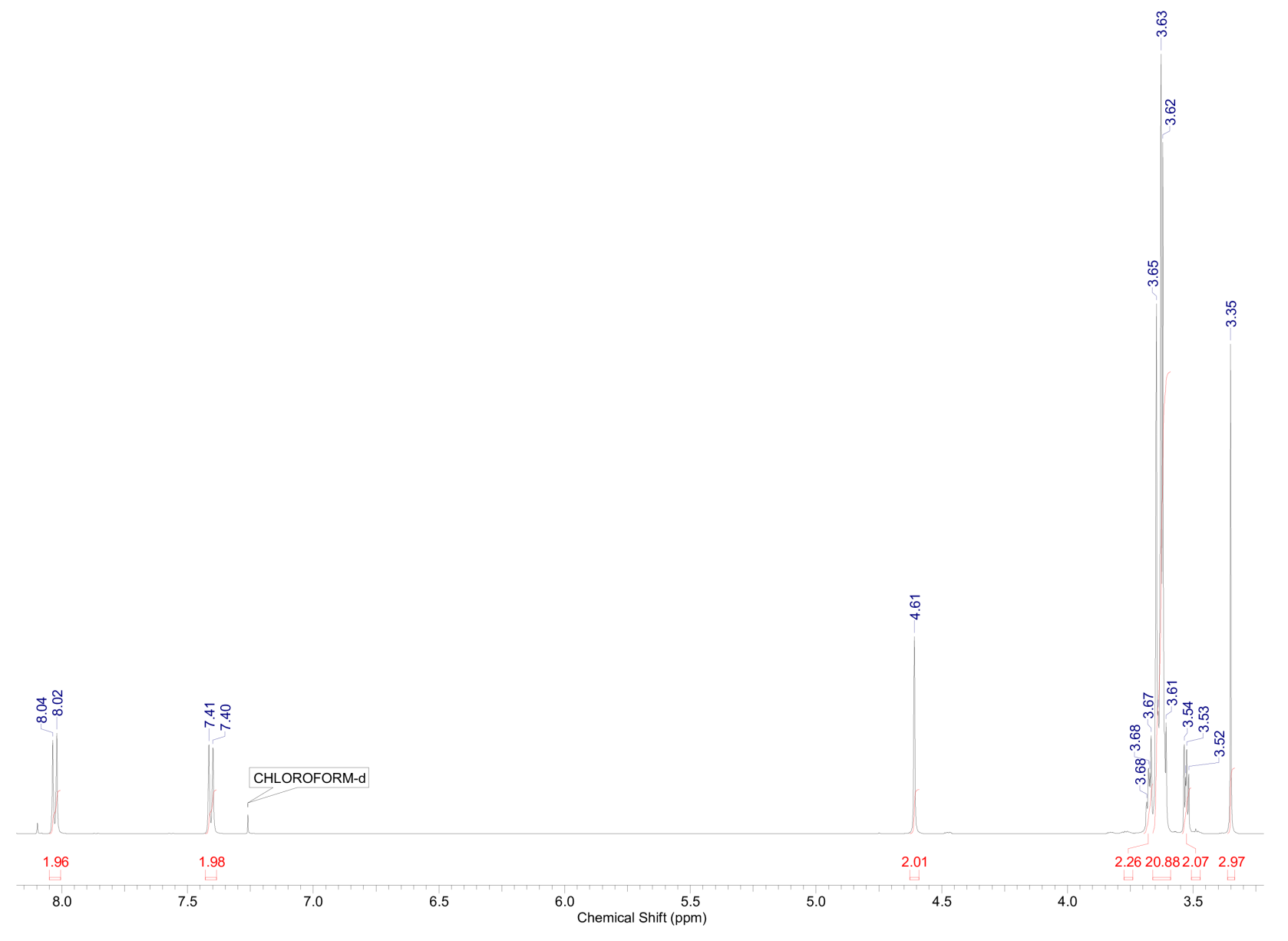

Figure S47. ${ }^{1} \mathrm{H}$ NMR spectrum $\left(500 \mathrm{MHz}, \mathrm{CDCl}_{3}\right)$ of $\mathrm{Ac} 2$. 


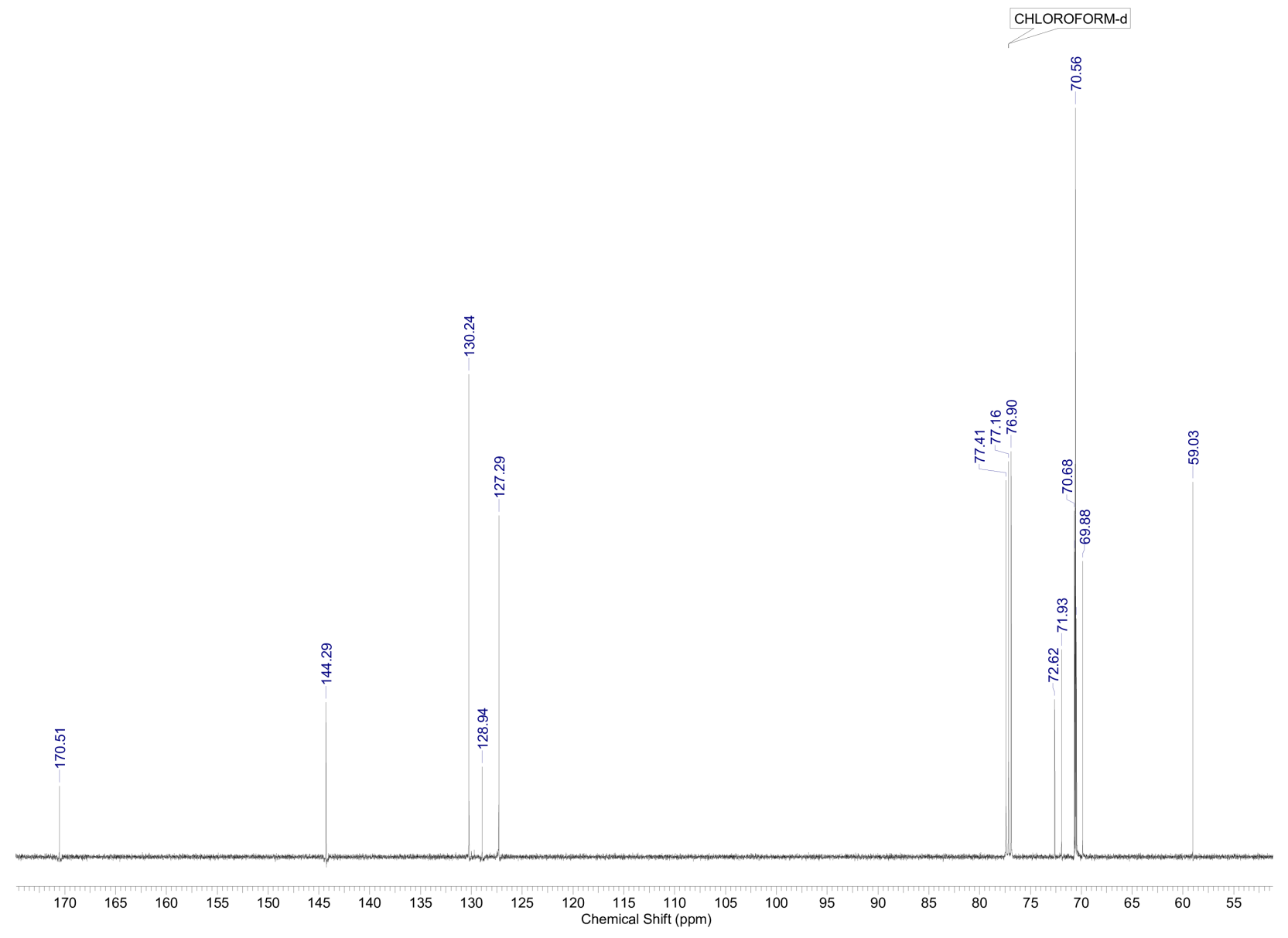

Figure $\mathrm{S} 48 .{ }^{13} \mathrm{C}\left\{{ }^{1} \mathrm{H}\right\} \mathrm{NMR}$ spectrum $\left(125 \mathrm{MHz}, \mathrm{CDCl}_{3}\right)$ of Ac2. 


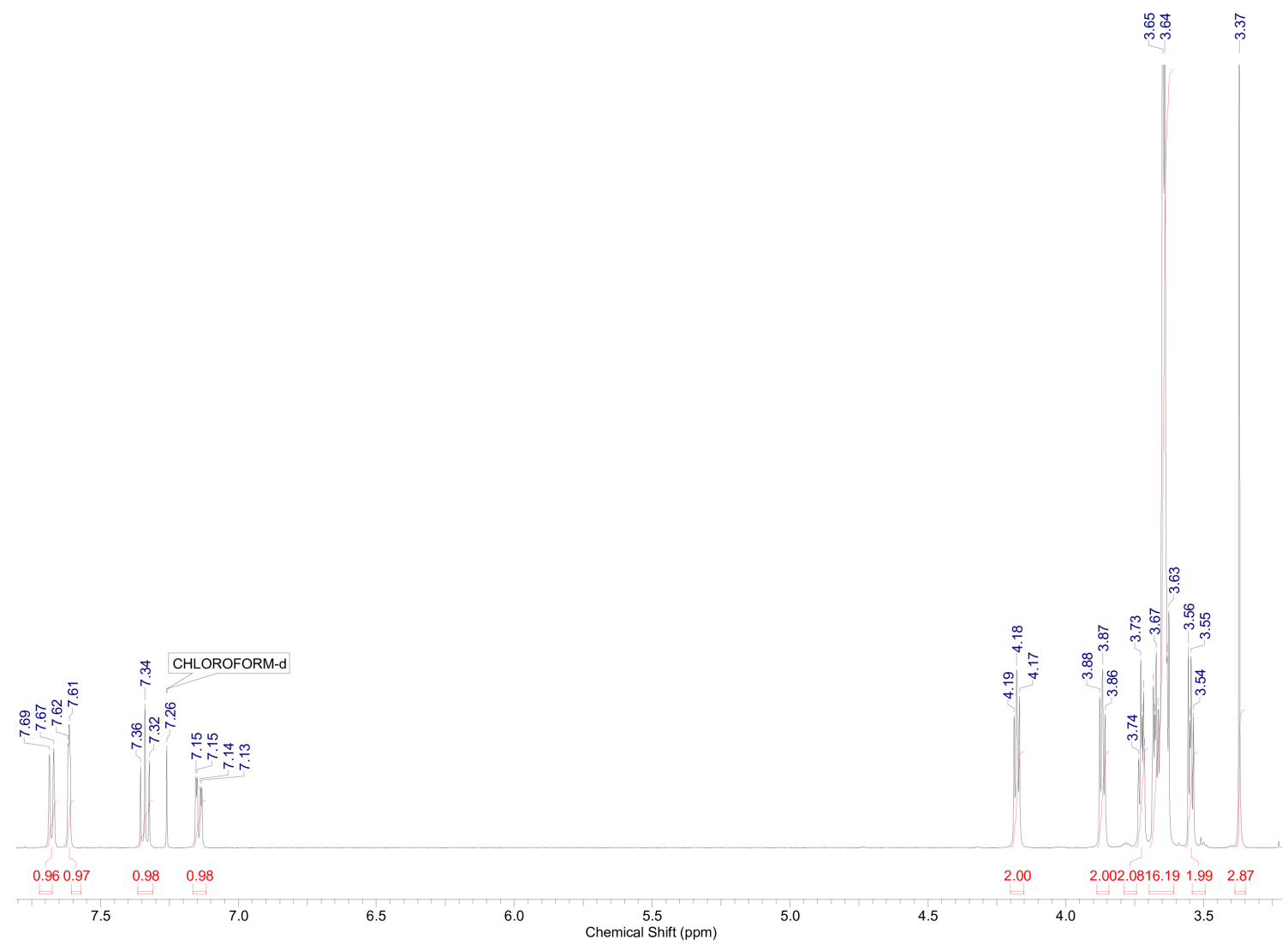

Figure S49. ${ }^{1} \mathrm{H}$ NMR spectrum $\left(500 \mathrm{MHz}, \mathrm{CDCl}_{3}\right)$ of $\mathrm{Ac3}$. 


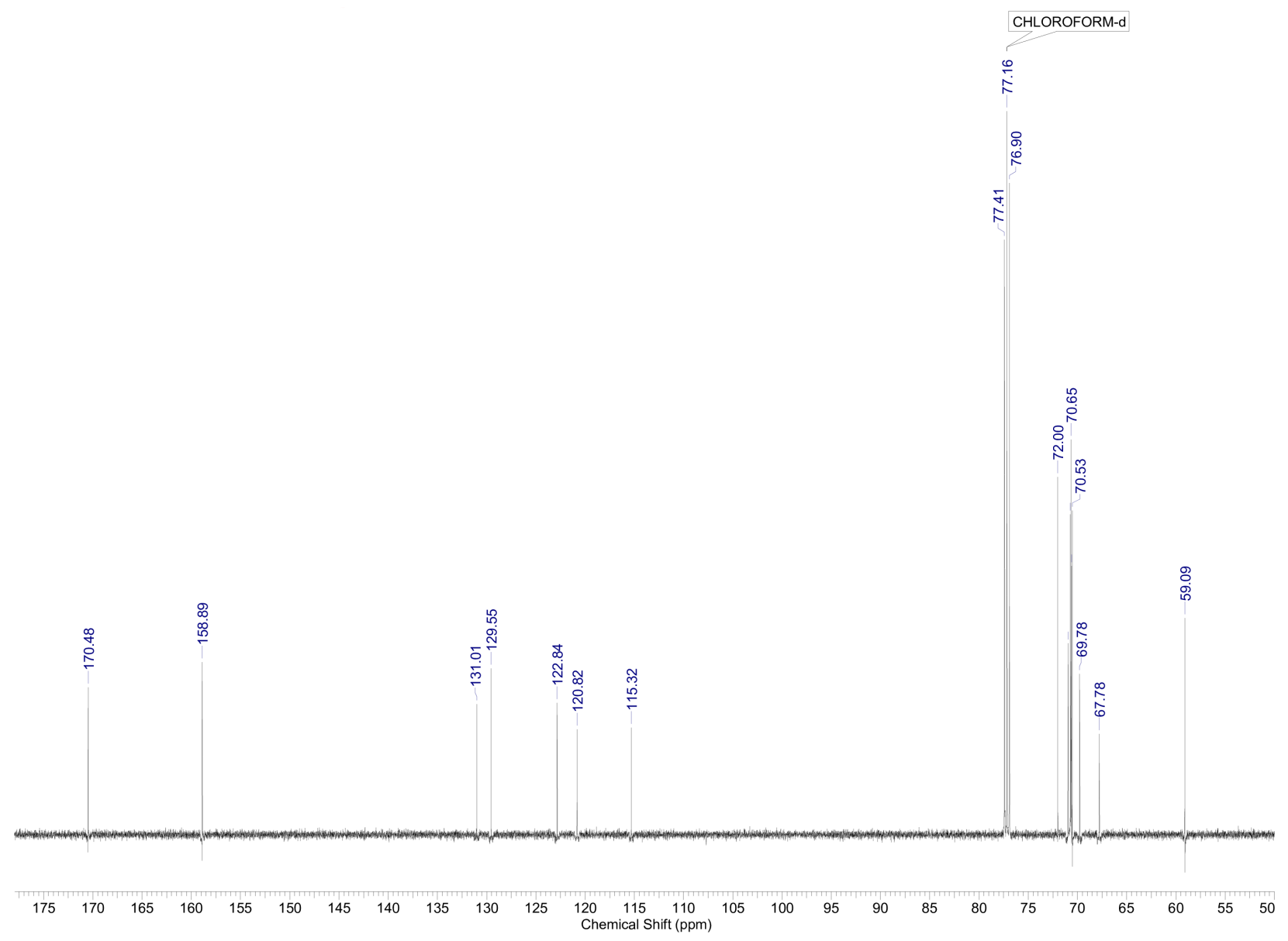

Figure S50. ${ }^{13} \mathrm{C}\left\{{ }^{1} \mathrm{H}\right\}$ NMR spectrum $\left(125 \mathrm{MHz}, \mathrm{CDCl}_{3}\right)$ of Ac3. 


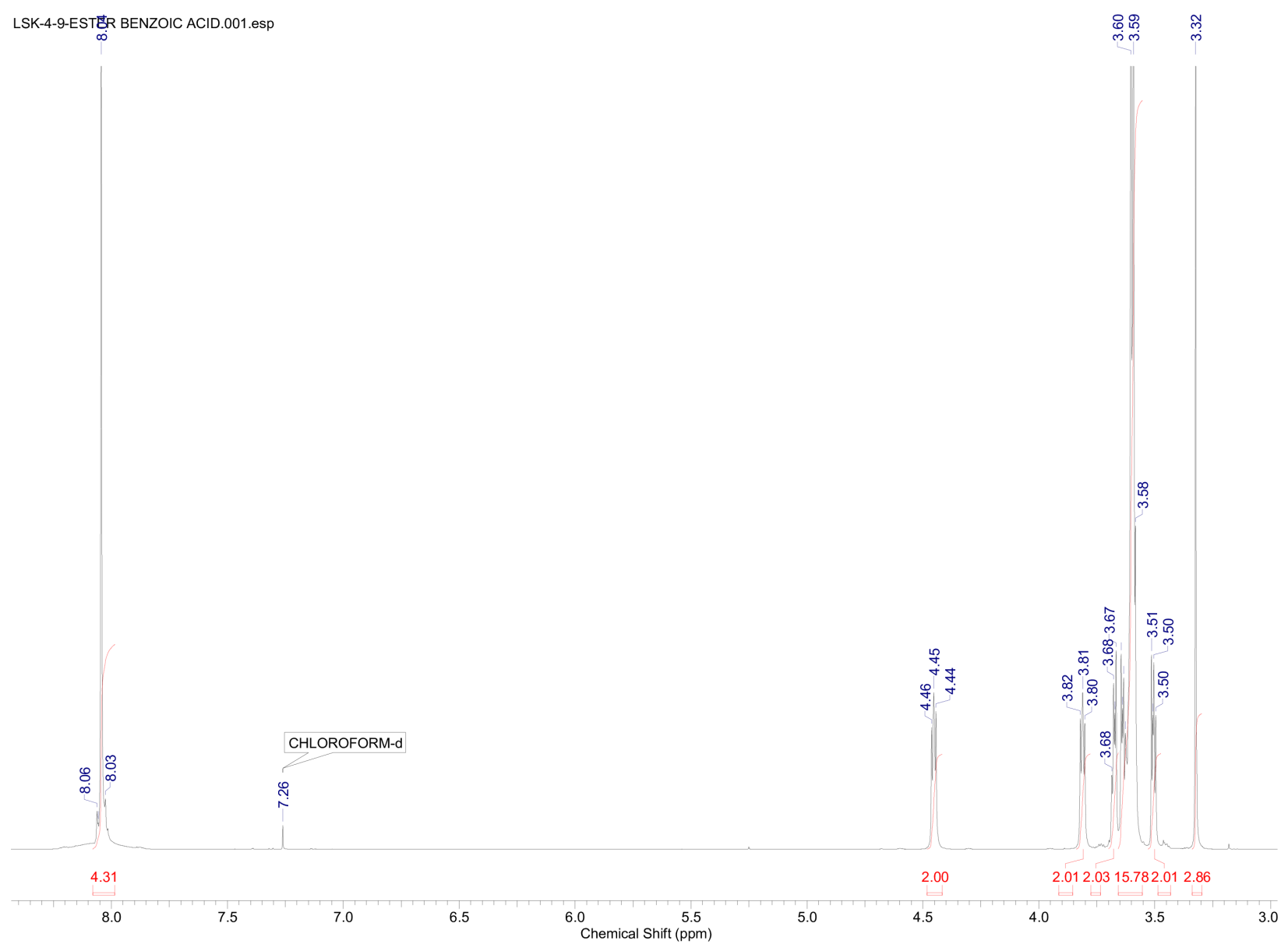

Figure S51. ${ }^{1} \mathrm{H}$ NMR spectrum $\left(500 \mathrm{MHz}, \mathrm{CDCl}_{3}\right)$ of Ac4. 


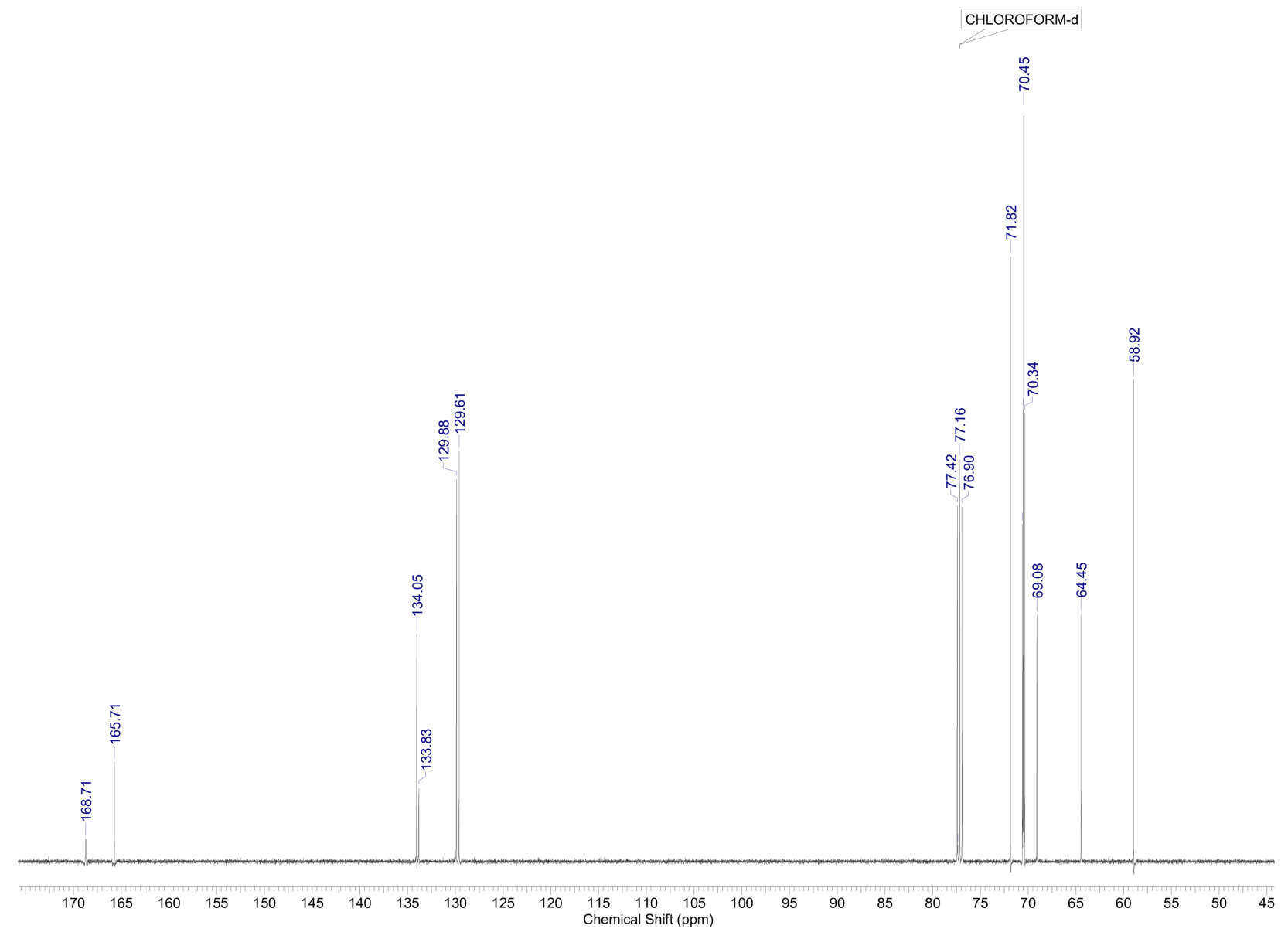

Figure $\mathrm{S} 52 .{ }^{13} \mathrm{C}\left\{{ }^{1} \mathrm{H}\right\}$ NMR spectrum $\left(125 \mathrm{MHz}, \mathrm{CDCl}_{3}\right)$ of Ac4. 


\section{Raw data for kinetics}

\begin{tabular}{rrrrr}
\hline Time $(\mathrm{min})$ & {$[$ An1 $](\mathrm{mM})$} & {$[$ Ac1 $](\mathrm{mM})$} & {$[\mathrm{EDU}](\mathrm{mM})$} & {$[\mathrm{EDC}](\mathrm{mM})$} \\
\hline 0 & 0 & 48.77 & 0 & \\
1.16 & 9.73 & 28.53 & 12.31 & 7.9 \\
2.17 & 12.71 & 23.07 & 16.02 & 4.51 \\
3.44 & 14.13 & 20.09 & 18.11 & 2.41 \\
5.96 & 15.07 & 18.93 & 20.21 & 1.08 \\
8.47 & 14.8 & 18.6 & 20.46 & 0.08 \\
10.99 & 14.78 & 19.17 & 21.1 & 0.38 \\
13.52 & 14.45 & 19.34 & 20.71 & 0 \\
49.81 & 11.23 & 25.94 & 21.04 & 0 \\
53.57 & 10.6 & 26.84 & 20.99 & 0 \\
139.39 & 5.76 & 36.59 & 20.94 & 0 \\
197.24 & 3.91 & 41.64 & 21.42 & 0 \\
213.16 & 3.27 & 42.81 & 21.58 & 0 \\
248.77 & 2.47 & 44.28 & 21.39 & 0 \\
264.57 & 2.12 & 44.73 & 21.72 & 0 \\
312.19 & 1.53 & 45.48 & 21.07 & 0 \\
\hline
\end{tabular}

Table S3. Concentration vs time data for $45 \mathrm{mM} \mathrm{Ac1}$ with 0.50 equiv EDC.

\begin{tabular}{rrrrr}
\hline Time $(\mathrm{min})$ & {$[\mathrm{An} 1](\mathrm{mM})$} & {$[\mathrm{Ac1}](\mathrm{mM})$} & {$[\mathrm{EDU}](\mathrm{mM})$} & {$[\mathrm{EDC}](\mathrm{mM})$} \\
\hline 0 & 0 & 49.05 & 0 & \\
1.17 & 13.81 & 20.09 & 18.34 & 14.56 \\
2.17 & 16.83 & 13.23 & 22.7 & 10.47 \\
3.42 & 18.47 & 10.35 & 25.66 & 8 \\
5.96 & 19.4 & 8.15 & 28.45 & 5.13 \\
8.47 & 19.59 & 8.24 & 30.24 & 3.51 \\
10.99 & 19.55 & 7.04 & 31.03 & 2.31 \\
13.51 & 19.01 & 8.9 & 32.17 & 1.54 \\
16.04 & 18.92 & 9.23 & 32.73 & 0.89 \\
18.56 & 18.37 & 9.71 & 33.1 & 0.41 \\
21.07 & 18.14 & 10.29 & 33.35 & 0.14 \\
23.59 & 17.58 & 11.43 & 33.68 & 0 \\
26.12 & 16.84 & 12.8 & 33.96 & 0 \\
28.62 & 16.55 & 13.83 & 34.2 & 0 \\
31.16 & 15.98 & 15.11 & 34.13 & 0 \\
38.72 & 15.14 & 18.29 & 34.39 & 0 \\
43.76 & 13.87 & 19.28 & 33.87 & 0 \\
51.32 & 13.15 & 21.11 & 33.81 & 0 \\
59.37 & 12.46 & 22.62 & 33.98 & 0 \\
67.37 & 11.5 & 24.08 & 33.85 & 0 \\
78.02 & 10.66 & 25.73 & 33.9 & 0 \\
81.27 & 10.77 & 26.13 & 34.31 & 0 \\
251.09 & 3.26 & 40.58 & 33.79 & 0 \\
315.57 & 2.15 & 43.29 & 33.88 & 0 \\
\hline & & & &
\end{tabular}

Table S4. Concentration vs time data for $45 \mathrm{mM}$ Ac1 with 0.75 equiv EDC. 


\begin{tabular}{rrrrr}
\hline Time $(\mathrm{min})$ & {$[$ An1 $](\mathrm{mM})$} & [Ac1] $(\mathrm{mM})$ & {$[\mathrm{EDU}](\mathrm{mM})$} & {$[\mathrm{EDC}](\mathrm{mM})$} \\
\hline 0 & 0 & 48.75 & 0 & \\
1.16 & 15.37 & 17.06 & 20.6 & 21.61 \\
2.17 & 18.5 & 9.27 & 24.63 & 16.61 \\
3.42 & 19.91 & 7.32 & 28.27 & 13.96 \\
5.94 & 20.57 & 5.82 & 31.85 & 10.65 \\
8.47 & 20.82 & 4.89 & 33.82 & 8.19 \\
10.99 & 20.72 & 5.27 & 36.01 & 6.4 \\
13.51 & 20.28 & 6.23 & 37.88 & 4.86 \\
54.96 & 13.94 & 19.86 & 43.37 & 0 \\
60.97 & 13.37 & 20.94 & 43.12 & 0 \\
150.26 & 6.83 & 33.95 & 43.2 & 0 \\
210.99 & 4.93 & 40.13 & 44.4 & 0 \\
226.69 & 4.42 & 41.75 & 44.83 & 0 \\
260.62 & 3.4 & 42.63 & 44.1 & 0 \\
277.06 & 3.08 & 43.89 & 44.35 & 0 \\
324.34 & 1.44 & 44.96 & 44.13 & 0 \\
\hline
\end{tabular}

Table S5. Concentration vs time data for $45 \mathrm{mM} \mathrm{Ac1}$ with 1.00 equiv EDC.

\begin{tabular}{|c|c|c|c|c|}
\hline Time (min) & [An1] (mM) & [Ac1] $(\mathrm{mM})$ & [EDU] (mM) & {$[\mathrm{EDC}](\mathrm{mM})$} \\
\hline 0 & 0 & 12.47 & 0 & \\
\hline 1.17 & 1.46 & 9.33 & 2.52 & 7.36 \\
\hline 1.67 & 1.9 & 8.39 & 3.39 & 6.54 \\
\hline 2.17 & 2.19 & 7.56 & 4.11 & 5.97 \\
\hline 3.42 & 2.88 & 6.45 & 5.42 & 4.73 \\
\hline 4.67 & 3.25 & 5.85 & 6.48 & 3.89 \\
\hline 5.94 & 3.22 & 5.52 & 6.97 & 3.18 \\
\hline 7.19 & 3.32 & 5.37 & 7.59 & 2.69 \\
\hline 8.44 & 3.3 & 5.45 & 7.88 & 2.16 \\
\hline 9.69 & 3.36 & 5.48 & 8.36 & 1.85 \\
\hline 10.94 & 3.26 & 5.49 & 8.67 & 1.59 \\
\hline 13.47 & 3.1 & 5.84 & 9.22 & 1.06 \\
\hline 35.49 & 1.27 & 9.86 & 10.39 & 0.09 \\
\hline 37.74 & 1.05 & 9.81 & 10.19 & 0.13 \\
\hline 49.05 & 0.57 & 11.06 & 10.47 & 0.05 \\
\hline 67.8 & 0.32 & 11.81 & 10.38 & 0.08 \\
\hline 70.07 & 0.18 & 11.76 & 10.17 & 0.02 \\
\hline
\end{tabular}

Table S6. Concentration vs time data for $12 \mathrm{mM}$ Ac1 with 0.75 equiv EDC. 


\begin{tabular}{rrrrr}
\hline Time $(\mathrm{min})$ & {$[\mathrm{An} 1](\mathrm{mM})$} & {$[\mathrm{Ac1}](\mathrm{mM})$} & {$[\mathrm{EDU}](\mathrm{mM})$} & {$[\mathrm{EDC}](\mathrm{mM})$} \\
\hline 0 & 0 & 24.74 & 0 & \\
1.19 & 4.03 & 16.46 & 5.52 & 5.48 \\
2.19 & 5.06 & 13.7 & 7.39 & 3.42 \\
3.47 & 6.17 & 11.88 & 9.27 & 2.36 \\
4.72 & 6.22 & 11.21 & 9.69 & 1.39 \\
5.97 & 6.8 & 10.7 & 10.63 & 1.06 \\
8.49 & 6.74 & 10.5 & 11.13 & 0.47 \\
11 & 6.51 & 11.1 & 11.33 & 0.16 \\
13.52 & 6.47 & 11.27 & 11.51 & 0.04 \\
63.65 & 2.55 & 19.17 & 11.61 & 0 \\
68.67 & 2.15 & 19.59 & 11.5 & 0 \\
73.7 & 2.18 & 20.13 & 11.75 & 0 \\
85.02 & 1.64 & 21.33 & 11.86 & 0 \\
98.35 & 1.07 & 22.17 & 11.77 & 0 \\
117.35 & 0.8 & 23.06 & 12.02 & 0 \\
\hline
\end{tabular}

Table S7. Concentration vs time data for $25 \mathrm{mM}$ Ac1 with 0.75 equiv EDC. 


\begin{tabular}{|c|c|c|c|c|}
\hline Time (min) & [An1] (mM) & [Ac1] $(\mathrm{mM})$ & {$[\mathrm{EDU}](\mathrm{mM})$} & {$[\mathrm{EDC}](\mathrm{mM})$} \\
\hline 0.00 & 0.00 & 33.14 & 0.00 & \\
\hline 1.16 & 9.20 & 13.35 & 12.60 & 17.16 \\
\hline 1.67 & 10.85 & 10.49 & 14.88 & 15.05 \\
\hline 2.17 & 11.60 & 8.58 & 16.38 & 13.47 \\
\hline 2.67 & 12.32 & 7.29 & 17.71 & 12.41 \\
\hline 3.17 & 12.37 & 6.75 & 18.53 & 11.47 \\
\hline 4.69 & 13.43 & 5.21 & 20.81 & 9.44 \\
\hline 5.94 & 13.26 & 4.86 & 21.89 & 8.10 \\
\hline 7.21 & 13.58 & 4.62 & 23.03 & 7.18 \\
\hline 8.46 & 13.25 & 4.88 & 23.83 & 6.20 \\
\hline 9.72 & 13.67 & 4.71 & 24.99 & 5.51 \\
\hline 10.97 & 13.19 & 5.15 & 25.41 & 4.70 \\
\hline 12.22 & 12.99 & 5.45 & 26.13 & 4.05 \\
\hline 13.47 & 13.04 & 5.33 & 26.78 & 3.40 \\
\hline 14.74 & 13.01 & 5.67 & 27.43 & 3.02 \\
\hline 15.99 & 12.55 & 6.18 & 27.66 & 2.53 \\
\hline 17.27 & 12.38 & 6.38 & 28.08 & 1.98 \\
\hline 19.79 & 12.29 & 7.02 & 29.18 & 1.42 \\
\hline 22.32 & 12.20 & 7.37 & 29.90 & 0.87 \\
\hline 26.09 & 11.45 & 8.61 & 30.28 & 0.20 \\
\hline 76.46 & 5.86 & 20.79 & 31.54 & 0.00 \\
\hline 81.24 & 5.04 & 20.72 & 30.64 & 0.00 \\
\hline 92.74 & 4.43 & 22.67 & 31.09 & 0.00 \\
\hline 112.27 & 3.74 & 24.69 & 31.17 & 0.00 \\
\hline 124.66 & 3.23 & 25.65 & 30.84 & 0.00 \\
\hline 157.84 & 1.75 & 28.67 & 31.42 & 0.00 \\
\hline 162.61 & 1.61 & 28.61 & 30.93 & 0.00 \\
\hline 262.77 & 0.12 & 31.62 & 31.23 & 0.00 \\
\hline 265.04 & 0.12 & 30.93 & 30.50 & 0.00 \\
\hline
\end{tabular}

Table S8. Concentration vs time data for $30 \mathrm{mM} \mathrm{Ac1}$ with 1.00 equiv EDC. 


\begin{tabular}{rrrrr}
\hline Time $(\mathrm{min})$ & {$[\mathrm{An} 1](\mathrm{mM})$} & {$[\mathrm{Ac1}](\mathrm{mM})$} & {$[\mathrm{EDU}](\mathrm{mM})$} & {$[\mathrm{EDC}](\mathrm{mM})$} \\
\hline 0.00 & 0.00 & 33.14 & 0.00 & \\
1.36 & 8.56 & 15.21 & 11.32 & 12.00 \\
1.86 & 9.59 & 12.93 & 12.97 & 10.11 \\
2.36 & 10.22 & 11.58 & 14.02 & 8.63 \\
3.61 & 11.47 & 8.79 & 16.61 & 6.56 \\
4.87 & 12.28 & 7.64 & 18.08 & 5.19 \\
6.12 & 12.14 & 7.46 & 18.83 & 4.15 \\
8.66 & 12.26 & 7.43 & 20.44 & 2.74 \\
11.17 & 12.09 & 7.52 & 21.47 & 1.71 \\
13.69 & 12.02 & 7.65 & 22.17 & 1.04 \\
16.19 & 11.94 & 8.33 & 22.91 & 0.66 \\
18.71 & 11.74 & 8.87 & 23.26 & 0.31 \\
21.22 & 10.94 & 9.84 & 23.32 & 0.05 \\
54.59 & 7.10 & 18.06 & 24.14 & 0.00 \\
56.86 & 6.81 & 17.97 & 23.72 & 0.00 \\
68.81 & 6.10 & 20.72 & 24.35 & 0.00 \\
74.84 & 5.24 & 21.00 & 23.53 & 0.00 \\
98.27 & 4.06 & 24.12 & 23.87 & 0.00 \\
103.06 & 3.86 & 24.33 & 23.83 & 0.00 \\
110.61 & 3.36 & 24.95 & 23.59 & 0.00 \\
137.62 & 2.57 & 28.10 & 24.32 & 0.00 \\
142.39 & 2.00 & 27.56 & 23.56 & 0.00 \\
239.04 & 0.47 & 31.62 & 24.37 & 0.00 \\
\hline & & & &
\end{tabular}

Table S9. Concentration vs time data for $30 \mathrm{mM} \mathrm{Ac1}$ with 0.75 equiv EDC. 


\begin{tabular}{rrrrr}
\hline Time $(\mathrm{min})$ & {$[$ An1 $](\mathrm{mM})$} & {$[$ Ac1 $](\mathrm{mM})$} & {$[\mathrm{EDU}](\mathrm{mM})$} & {$[\mathrm{EDC}](\mathrm{mM})$} \\
\hline 0.00 & 0.00 & 33.14 & 0.00 & \\
1.19 & 5.47 & 21.09 & 7.12 & 7.61 \\
1.71 & 6.89 & 18.62 & 8.98 & 6.08 \\
2.21 & 7.34 & 17.01 & 9.88 & 4.74 \\
2.71 & 7.98 & 15.60 & 10.88 & 3.91 \\
3.46 & 8.95 & 14.10 & 12.20 & 2.99 \\
4.71 & 9.54 & 13.10 & 13.37 & 2.03 \\
5.97 & 9.66 & 12.75 & 13.72 & 1.10 \\
8.47 & 9.98 & 12.17 & 14.77 & 0.39 \\
10.99 & 9.79 & 12.17 & 15.22 & 0.00 \\
13.49 & 9.68 & 12.90 & 15.49 & 0.00 \\
16.01 & 9.20 & 13.52 & 15.50 & 0.00 \\
18.52 & 8.94 & 14.28 & 15.51 & 0.00 \\
33.86 & 7.29 & 17.73 & 15.73 & 0.00 \\
51.92 & 5.77 & 20.57 & 15.70 & 0.00 \\
55.44 & 5.81 & 20.87 & 15.87 & 0.00 \\
87.64 & 3.71 & 25.16 & 15.77 & 0.00 \\
93.69 & 3.78 & 25.59 & 15.70 & 0.00 \\
100.01 & 3.02 & 25.82 & 15.49 & 0.00 \\
120.26 & 2.52 & 28.08 & 15.96 & 0.00 \\
125.02 & 2.22 & 27.99 & 15.84 & 0.00 \\
216.92 & 0.31 & 31.62 & 15.53 & 0.00 \\
\hline
\end{tabular}

Table S10. Concentration vs time data for $30 \mathrm{mM}$ Ac1 with 0.50 equiv EDC. 


\begin{tabular}{rrrrr}
\hline Time $(\mathrm{min})$ & {$[\mathrm{An} 2](\mathrm{mM})$} & {$[\mathrm{Ac} 2](\mathrm{mM})$} & {$[\mathrm{EDU}](\mathrm{mM})$} & {$[\mathrm{EDC}](\mathrm{mM})$} \\
\hline 0.00 & 0.00 & 47.09 & 0.00 & \\
1.05 & 9.75 & 27.24 & 12.42 & 8.67 \\
2.05 & 11.53 & 23.30 & 16.18 & 4.58 \\
3.32 & 11.39 & 23.57 & 18.60 & 2.03 \\
4.59 & 10.70 & 25.35 & 20.21 & 0.79 \\
5.89 & 9.45 & 27.81 & 20.71 & 0.16 \\
7.14 & 8.08 & 30.39 & 21.05 & 0.00 \\
8.39 & 7.00 & 32.78 & 21.34 & 0.00 \\
9.67 & 5.71 & 34.49 & 21.08 & 0.00 \\
10.94 & 4.71 & 36.17 & 20.81 & 0.00 \\
12.19 & 4.12 & 37.73 & 21.26 & 0.00 \\
13.44 & 3.69 & 39.29 & 21.36 & 0.00 \\
14.70 & 3.07 & 40.16 & 21.34 & 0.00 \\
15.97 & 2.58 & 40.94 & 21.27 & 0.00 \\
17.22 & 2.17 & 41.99 & 20.97 & 0.00 \\
18.49 & 1.91 & 42.69 & 21.36 & 0.00 \\
19.74 & 1.72 & 43.43 & 21.29 & 0.00 \\
21.00 & 1.38 & 43.79 & 21.10 & 0.00 \\
22.27 & 1.10 & 44.18 & 21.15 & 0.00 \\
23.54 & 0.98 & 44.55 & 21.11 & 0.00 \\
24.80 & 0.77 & 44.81 & 21.07 & 0.00 \\
26.65 & 0.69 & 45.15 & 21.00 & 0.00 \\
27.90 & 0.57 & 45.71 & 21.23 & 0.00 \\
\hline
\end{tabular}

Table S11. Concentration vs time data for $45 \mathrm{mM} \mathrm{Ac2}$ with 0.50 equiv EDC. 


\begin{tabular}{rrrrr}
\hline Time $(\mathrm{min})$ & {$[\mathrm{An} 2](\mathrm{mM})$} & {$[\mathrm{Ac} 2](\mathrm{mM})$} & {$[\mathrm{EDU}](\mathrm{mM})$} & {$[\mathrm{EDC}](\mathrm{mM})$} \\
\hline 0.00 & 0.00 & 47.09 & 0.00 & \\
1.07 & 13.80 & 19.49 & 18.53 & 15.22 \\
2.09 & 14.91 & 16.28 & 23.86 & 10.08 \\
3.35 & 14.41 & 16.94 & 27.68 & 6.18 \\
4.60 & 13.34 & 18.92 & 30.46 & 3.59 \\
5.85 & 12.35 & 21.48 & 32.36 & 1.93 \\
7.10 & 11.16 & 24.17 & 33.24 & 0.87 \\
8.37 & 9.97 & 27.09 & 33.88 & 0.45 \\
9.64 & 8.11 & 29.82 & 34.02 & 0.07 \\
10.90 & 7.18 & 32.06 & 34.17 & 0.13 \\
12.17 & 5.78 & 33.98 & 34.23 & 0.11 \\
13.42 & 5.07 & 35.97 & 34.31 & 0.06 \\
14.69 & 4.17 & 37.26 & 34.16 & 0.07 \\
15.94 & 3.56 & 38.64 & 34.19 & 0.03 \\
17.19 & 3.15 & 39.86 & 34.22 & 0.02 \\
18.45 & 2.57 & 41.00 & 34.25 & 0.02 \\
19.72 & 2.21 & 41.34 & 34.25 & 0.13 \\
20.97 & 1.88 & 42.06 & 34.60 & 0.11 \\
22.22 & 1.75 & 42.86 & 34.28 & 0.12 \\
23.49 & 1.40 & 43.44 & 34.19 & 0.06 \\
24.74 & 1.03 & 43.58 & 34.03 & 0.00 \\
25.99 & 1.09 & 44.12 & 34.23 & 0.03 \\
27.25 & 0.74 & 44.46 & 33.97 & 0.03 \\
\hline
\end{tabular}

Table S12. Concentration vs time data for $45 \mathrm{mM} \mathrm{Ac2}$ with 0.75 equiv EDC. 


\begin{tabular}{rrrrr}
\hline Time $(\mathrm{min})$ & {$[\mathrm{An} 2](\mathrm{mM})$} & {$[\mathrm{Ac} 2](\mathrm{mM})$} & {$[\mathrm{EDU}](\mathrm{mM})$} & {$[\mathrm{EDC}](\mathrm{mM})$} \\
\hline 0.00 & 0.00 & 47.09 & 0.00 & \\
1.19 & 15.30 & 15.29 & 22.04 & 20.93 \\
2.19 & 16.27 & 13.20 & 27.84 & 15.21 \\
3.44 & 15.89 & 14.19 & 33.54 & 10.24 \\
4.69 & 14.82 & 16.17 & 36.98 & 6.36 \\
5.97 & 13.72 & 18.62 & 39.89 & 3.82 \\
7.24 & 12.80 & 21.11 & 41.84 & 2.00 \\
8.49 & 11.25 & 23.79 & 42.90 & 1.03 \\
9.75 & 9.93 & 26.75 & 43.44 & 0.47 \\
11.02 & 8.28 & 29.36 & 43.75 & 0.23 \\
12.27 & 6.97 & 31.73 & 43.71 & 0.10 \\
13.54 & 5.57 & 33.77 & 43.79 & 0.12 \\
14.80 & 4.90 & 35.79 & 43.94 & 0.07 \\
16.05 & 4.09 & 37.10 & 43.85 & 0.00 \\
17.34 & 3.47 & 38.36 & 43.72 & 0.04 \\
18.59 & 3.06 & 39.65 & 43.99 & 0.12 \\
19.85 & 2.54 & 40.55 & 43.79 & 0.04 \\
21.12 & 2.11 & 41.25 & 43.65 & 0.03 \\
22.37 & 1.82 & 41.94 & 43.88 & 0.10 \\
23.64 & 1.49 & 42.36 & 43.74 & 0.17 \\
24.89 & 1.32 & 43.10 & 43.75 & 0.03 \\
26.15 & 1.09 & 43.43 & 43.79 & 0.08 \\
27.40 & 0.94 & 43.80 & 43.76 & 0.09 \\
\hline
\end{tabular}

Table S13. Concentration vs time data for $45 \mathrm{mM}$ Ac2 with 1.00 equiv EDC.

\begin{tabular}{rrrrr}
\hline Time $(\mathrm{min})$ & {$[\mathrm{An} 2](\mathrm{mM})$} & {$[\mathrm{Ac} 2](\mathrm{mM})$} & {$[\mathrm{EDU}](\mathrm{mM})$} & {$[\mathrm{EDC}](\mathrm{mM})$} \\
\hline 0.00 & 0.00 & 25.47 & 0.00 & \\
1.00 & 4.16 & 15.53 & 6.92 & 11.71 \\
2.02 & 5.01 & 13.31 & 10.32 & 8.08 \\
3.27 & 4.88 & 13.59 & 13.78 & 5.37 \\
4.57 & 4.42 & 14.84 & 16.01 & 3.31 \\
5.82 & 3.64 & 16.44 & 17.17 & 1.81 \\
7.07 & 2.86 & 17.97 & 18.05 & 1.05 \\
8.34 & 2.18 & 19.17 & 18.52 & 0.47 \\
9.59 & 1.61 & 20.31 & 18.73 & 0.27 \\
10.85 & 1.18 & 21.08 & 18.78 & 0.08 \\
12.10 & 0.92 & 21.90 & 18.97 & 0.04 \\
13.35 & 0.53 & 22.41 & 19.00 & 0.04 \\
14.62 & 0.35 & 22.67 & 18.91 & 0.01 \\
15.87 & 0.24 & 23.03 & 19.03 & 0.00 \\
17.14 & 0.20 & 23.25 & 19.19 & 0.03 \\
18.39 & 0.14 & 23.28 & 19.18 & 0.00 \\
\hline
\end{tabular}

Table S14. Concentration vs time data for $25 \mathrm{mM} \mathrm{Ac2}$ with 0.75 equiv EDC. 


\begin{tabular}{rrrrr}
\hline Time $(\mathrm{min})$ & {$[\mathrm{An} 2](\mathrm{mM})$} & {$[\mathrm{Ac} 2](\mathrm{mM})$} & {$[\mathrm{EDU}](\mathrm{mM})$} & {$[\mathrm{EDC}](\mathrm{mM})$} \\
\hline 0.00 & 0.00 & 12.86 & 0.00 & \\
1.12 & 1.31 & 9.71 & 2.99 & 7.00 \\
1.62 & 1.35 & 9.30 & 3.78 & 6.09 \\
2.14 & 1.41 & 9.15 & 4.45 & 5.27 \\
2.64 & 1.49 & 9.35 & 5.50 & 4.69 \\
3.39 & 1.28 & 9.42 & 6.02 & 3.67 \\
4.65 & 1.15 & 9.92 & 7.41 & 2.55 \\
5.90 & 0.90 & 10.26 & 8.31 & 1.74 \\
7.15 & 0.69 & 10.62 & 8.85 & 1.21 \\
8.42 & 0.52 & 11.16 & 9.39 & 0.72 \\
9.69 & 0.35 & 11.36 & 9.68 & 0.53 \\
10.94 & 0.31 & 11.70 & 9.84 & 0.30 \\
12.20 & 0.20 & 11.78 & 9.98 & 0.24 \\
13.47 & 0.04 & 11.73 & 9.76 & 0.07 \\
\hline
\end{tabular}

Table S15. Concentration vs time data for $12 \mathrm{mM} \mathrm{Ac2}$ with 0.75 equiv EDC.

\begin{tabular}{rrrrr}
\hline Time $(\mathrm{min})$ & {$[\mathrm{An} 2](\mathrm{mM})$} & {$[\mathrm{Ac} 2](\mathrm{mM})$} & {$[\mathrm{EDU}](\mathrm{mM})$} & {$[\mathrm{EDC}](\mathrm{mM})$} \\
\hline 0.00 & 0.00 & 30.72 & 0.00 & \\
1.11 & 8.03 & 13.77 & 12.11 & 19.04 \\
1.61 & 8.78 & 12.18 & 15.03 & 16.38 \\
2.11 & 8.96 & 11.81 & 17.26 & 14.14 \\
2.61 & 8.86 & 12.05 & 19.35 & 12.19 \\
3.11 & 8.91 & 12.20 & 21.22 & 10.53 \\
4.62 & 8.00 & 14.04 & 25.70 & 6.25 \\
5.89 & 6.96 & 15.65 & 28.17 & 3.90 \\
7.14 & 5.93 & 17.39 & 29.99 & 2.10 \\
8.41 & 4.76 & 19.49 & 31.14 & 1.25 \\
9.67 & 3.86 & 21.54 & 31.87 & 0.65 \\
10.92 & 3.01 & 23.09 & 32.02 & 0.33 \\
12.17 & 2.22 & 24.57 & 32.15 & 0.12 \\
13.44 & 1.70 & 25.79 & 32.23 & 0.05 \\
14.69 & 1.32 & 26.73 & 32.47 & 0.03 \\
15.96 & 0.89 & 27.50 & 32.30 & 0.03 \\
17.22 & 0.65 & 27.80 & 32.22 & 0.04 \\
18.47 & 0.41 & 28.23 & 32.40 & 0.01 \\
19.74 & 0.27 & 28.61 & 32.31 & 0.00 \\
21.01 & 0.17 & 28.97 & 32.33 & 0.04 \\
22.29 & 0.14 & 28.94 & 32.28 & 0.00 \\
\hline & & & &
\end{tabular}

Table S16. Concentration vs time data for $30 \mathrm{mM} \mathrm{Ac2}$ with 1.00 equiv EDC. 


\begin{tabular}{rrrrr}
\hline Time $(\mathrm{min})$ & {$[\mathrm{An} 2](\mathrm{mM})$} & {$[\mathrm{Ac} 2](\mathrm{mM})$} & {$[\mathrm{EDU}](\mathrm{mM})$} & {$[\mathrm{EDC}](\mathrm{mM})$} \\
\hline 0.00 & 0.00 & 30.72 & 0.00 & \\
1.12 & 6.63 & 17.03 & 9.81 & 11.06 \\
1.62 & 7.10 & 15.51 & 11.89 & 8.97 \\
2.12 & 7.27 & 15.18 & 13.30 & 7.25 \\
2.62 & 7.24 & 15.09 & 14.82 & 5.97 \\
3.14 & 7.25 & 15.50 & 16.09 & 4.87 \\
3.66 & 6.82 & 16.01 & 17.24 & 3.97 \\
4.66 & 6.11 & 17.51 & 18.57 & 2.42 \\
5.91 & 5.06 & 19.32 & 19.82 & 1.33 \\
7.17 & 4.08 & 21.33 & 20.60 & 0.66 \\
8.44 & 3.14 & 23.06 & 21.11 & 0.33 \\
9.69 & 2.59 & 24.98 & 21.47 & 0.29 \\
10.97 & 1.83 & 26.03 & 21.26 & 0.14 \\
12.22 & 1.40 & 27.06 & 21.54 & 0.22 \\
13.49 & 0.98 & 27.72 & 21.37 & 0.04 \\
14.74 & 0.77 & 28.41 & 21.45 & 0.09 \\
16.01 & 0.49 & 28.73 & 21.28 & 0.00 \\
17.27 & 0.39 & 29.13 & 21.33 & 0.04 \\
18.54 & 0.17 & 29.18 & 21.22 & 0.03 \\
19.79 & 0.08 & 29.39 & 21.09 & 0.00 \\
\hline
\end{tabular}

Table S17. Concentration vs time data for $30 \mathrm{mM} \mathrm{Ac2}$ with 0.75 equiv EDC.

\begin{tabular}{rrrrr}
\hline Time $(\mathrm{min})$ & {$[\mathrm{An} 2](\mathrm{mM})$} & {$[\mathrm{Ac} 2](\mathrm{mM})$} & {$[\mathrm{EDU}](\mathrm{mM})$} & {$[\mathrm{EDC}](\mathrm{mM})$} \\
\hline 0.00 & 0.00 & 30.72 & 0.00 & \\
1.42 & 5.20 & 19.65 & 7.64 & 7.67 \\
1.92 & 5.69 & 18.59 & 9.05 & 6.06 \\
2.42 & 5.87 & 17.88 & 10.14 & 4.77 \\
2.92 & 5.87 & 17.81 & 11.22 & 3.69 \\
3.42 & 5.80 & 18.15 & 12.14 & 2.98 \\
3.92 & 5.70 & 18.63 & 12.97 & 2.42 \\
4.42 & 5.48 & 19.22 & 13.66 & 2.05 \\
4.92 & 5.00 & 19.79 & 14.05 & 1.49 \\
6.21 & 4.25 & 21.72 & 15.13 & 0.88 \\
7.47 & 3.40 & 23.33 & 15.32 & 0.31 \\
8.72 & 2.48 & 24.92 & 15.45 & 0.19 \\
9.97 & 1.87 & 26.10 & 15.42 & 0.04 \\
11.22 & 1.40 & 26.97 & 15.45 & 0.03 \\
12.49 & 1.08 & 27.95 & 15.58 & 0.07 \\
13.74 & 0.86 & 28.64 & 15.65 & 0.02 \\
15.01 & 0.58 & 28.95 & 15.67 & 0.09 \\
16.26 & 0.33 & 29.13 & 15.53 & 0.01 \\
17.52 & 0.36 & 29.54 & 15.68 & 0.17 \\
18.77 & 0.32 & 29.66 & 15.73 & 0.15 \\
\hline
\end{tabular}

Table S18. Concentration vs time data for $30 \mathrm{mM}$ Ac2 with 0.5 equiv EDC. 


\begin{tabular}{rrrrr}
\hline Time $(\min )$ & {$[\mathrm{An} 3](\mathrm{mM})$} & {$[\mathrm{Ac} 3](\mathrm{mM})$} & {$[\mathrm{EDU}](\mathrm{mM})$} & {$[\mathrm{EDC}](\mathrm{mM})$} \\
\hline 0 & 0 & 46.83 & 0 & \\
1.07 & 8.445 & 29.94 & 11.12 & 8.6 \\
1.32 & 9.165 & 28.23 & 12.19 & 7.34 \\
1.57 & 9.09 & 27.36 & 13.22 & 6.5 \\
1.82 & 9.435 & 26.88 & 13.91 & 5.55 \\
2.07 & 9.51 & 26.19 & 14.59 & 4.73 \\
2.57 & 9.735 & 26.37 & 15.73 & 3.59 \\
3.34 & 9.795 & 26.7 & 17.44 & 2.38 \\
4.6 & 8.37 & 28.38 & 18.39 & 0.87 \\
5.85 & 6.915 & 30.63 & 19.16 & 0.28 \\
7.12 & 6.195 & 33.42 & 19.55 & 0 \\
8.4 & 4.8 & 35.94 & 19.84 & 0 \\
9.69 & 3.63 & 38.13 & 19.8 & 0 \\
10.94 & 3.27 & 39.9 & 19.95 & 0 \\
12.2 & 2.25 & 40.77 & 19.81 & 0 \\
13.47 & 1.59 & 42.03 & 19.87 & 0 \\
14.75 & 0.915 & 43.11 & 19.74 & 0 \\
16.02 & 0.675 & 43.65 & 19.6 & 0 \\
18.55 & 0.3 & 45.09 & 19.95 & 0 \\
\hline
\end{tabular}

Table S19. Concentration vs time data for $45 \mathrm{mM} \mathrm{Ac3}$ with 0.50 equiv EDC.

\begin{tabular}{rrrrr}
\hline Time $(\mathrm{min})$ & {$[\mathrm{An} 3](\mathrm{mM})$} & {$[\mathrm{Ac} 3](\mathrm{mM})$} & {$[\mathrm{EDU}](\mathrm{mM})$} & {$[\mathrm{EDC}](\mathrm{mM})$} \\
\hline 0 & 0 & 46.83 & 0 & \\
1.12 & 10.965 & 23.07 & 15.75 & 15.4 \\
1.37 & 11.67 & 21.51 & 17.38 & 13.88 \\
1.62 & 12.255 & 21.06 & 18.77 & 12.63 \\
1.87 & 12.45 & 20.13 & 19.81 & 11.49 \\
2.12 & 12.735 & 19.86 & 20.87 & 10.48 \\
2.64 & 12.735 & 19.53 & 22.51 & 8.58 \\
3.39 & 12.57 & 19.92 & 25.02 & 6.39 \\
4.64 & 11.505 & 22.08 & 27.69 & 3.78 \\
5.9 & 10.32 & 24.75 & 29.44 & 2.02 \\
7.15 & 8.895 & 27.3 & 30.57 & 0.98 \\
8.42 & 7.68 & 30.45 & 31.41 & 0.36 \\
9.69 & 6.15 & 32.46 & 31.57 & 0 \\
10.95 & 4.845 & 35.13 & 31.62 & 0 \\
12.2 & 3.855 & 37.14 & 31.62 & 0 \\
13.45 & 3.15 & 39 & 31.76 & 0 \\
14.72 & 2.31 & 40.5 & 31.64 & 0 \\
15.97 & 1.605 & 41.97 & 31.7 & 0 \\
17.24 & 0.765 & 42.6 & 31.78 & 0 \\
18.49 & 0.735 & 43.41 & 31.62 & 0 \\
\hline
\end{tabular}

Table S20. Concentration vs time data for $45 \mathrm{mM} \mathrm{Ac3}$ with 0.75 equiv EDC. 


\begin{tabular}{rrrrr}
\hline Time $(\mathrm{min})$ & {$[\mathrm{An} 3](\mathrm{mM})$} & {$[\mathrm{Ac} 3](\mathrm{mM})$} & {$[\mathrm{EDU}](\mathrm{mM})$} & {$[\mathrm{EDC}](\mathrm{mM})$} \\
\hline 0 & 0 & 46.83 & 0 & \\
1.12 & 13.425 & 18.96 & 18.78 & 23.57 \\
1.62 & 14.13 & 16.2 & 22.11 & 20.17 \\
2.14 & 14.52 & 15.78 & 24.89 & 17.51 \\
2.64 & 14.43 & 15.36 & 27.72 & 15.29 \\
3.14 & 14.295 & 15.96 & 30.11 & 13.17 \\
4.64 & 13.5 & 17.67 & 34.85 & 8.13 \\
5.9 & 12.285 & 20.1 & 38.12 & 5.08 \\
7.15 & 11.115 & 22.56 & 40.6 & 2.91 \\
8.4 & 9.735 & 25.29 & 41.53 & 1.55 \\
9.67 & 8.475 & 27.99 & 42.67 & 0.56 \\
10.92 & 6.75 & 30.99 & 43.16 & 0.2 \\
12.19 & 5.52 & 33.3 & 43.41 & 0 \\
13.47 & 4.47 & 35.7 & 43.62 & 0 \\
14.75 & 3.735 & 37.89 & 43.67 & 0 \\
16 & 2.61 & 39.42 & 43.51 & 0 \\
18.52 & 1.425 & 42 & 43.62 & 0 \\
21.04 & 0.495 & 43.41 & 43.53 & 0 \\
23.54 & 0.525 & 44.46 & 43.64 & 0 \\
\hline
\end{tabular}

Table S21. Concentration vs time data for $45 \mathrm{mM} \mathrm{Ac3}$ with 1.00 equiv EDC.

\begin{tabular}{rrrrr}
\hline Time $(\mathrm{min})$ & {$[\mathrm{An} 3](\mathrm{mM})$} & {$[\mathrm{Ac} 3](\mathrm{mM})$} & {$[\mathrm{EDU}](\mathrm{mM})$} & {$[\mathrm{EDC}](\mathrm{mM})$} \\
\hline 0 & 0 & 23.67 & 0 & \\
1.07 & 2.43 & 17.91 & 4.72 & 7.84 \\
1.32 & 2.43 & 17.58 & 5.23 & 6.97 \\
1.57 & 3.15 & 17.25 & 6.26 & 6.42 \\
1.84 & 3.045 & 17.1 & 6.84 & 5.85 \\
2.09 & 2.895 & 16.98 & 7.26 & 5.16 \\
2.59 & 2.745 & 17.31 & 8.26 & 4.25 \\
3.35 & 2.82 & 17.85 & 9.61 & 3.07 \\
4.62 & 2.325 & 19.26 & 11.24 & 1.83 \\
5.89 & 1.11 & 19.95 & 11.8 & 0.79 \\
7.15 & 0.99 & 21.27 & 12.49 & 0.51 \\
8.4 & 0.135 & 21.72 & 12.47 & 0.03 \\
\hline
\end{tabular}

Table S22. Concentration vs time data for $25 \mathrm{mM} \mathrm{Ac3}$ with 0.75 equiv EDC. 


\begin{tabular}{rrrrr}
\hline Time $(\mathrm{min})$ & {$[\mathrm{An} 3](\mathrm{mM})$} & {$[\mathrm{Ac} 3](\mathrm{mM})$} & {$[\mathrm{EDU}](\mathrm{mM})$} & {$[\mathrm{EDC}](\mathrm{mM})$} \\
\hline 0 & 0 & 11.43 & 0 & \\
1.12 & 0.53 & 9.63 & 2.04 & 5.88 \\
1.62 & 0.62 & 9.78 & 2.82 & 5.21 \\
2.12 & 0.56 & 9.63 & 3.31 & 4.49 \\
2.62 & 0.48 & 9.39 & 3.8 & 3.89 \\
3.12 & 0.65 & 9.75 & 4.51 & 3.47 \\
3.64 & 0.38 & 9.93 & 4.87 & 3.01 \\
4.66 & 0.21 & 9.9 & 5.64 & 2.17 \\
5.91 & 0.26 & 10.59 & 6.6 & 1.62 \\
7.17 & 0.09 & 10.62 & 7.1 & 1.09 \\
\hline
\end{tabular}

Table S23. Concentration vs time data for $12 \mathrm{mM} \mathrm{Ac3}$ with 0.75 equiv EDC.

\begin{tabular}{rrrrr}
\hline Time $(\mathrm{min})$ & {$[\mathrm{An} 3](\mathrm{mM})$} & {$[\mathrm{Ac} 3](\mathrm{mM})$} & {$[\mathrm{EDU}](\mathrm{mM})$} & {$[\mathrm{EDC}](\mathrm{mM})$} \\
\hline 0.00 & 0 & 33.12 & 0 & \\
1.04 & 6.465 & 16.59 & 11.11 & 18.89 \\
1.56 & 7.98 & 14.46 & 14.37 & 16.08 \\
2.06 & 8.55 & 13.68 & 16.67 & 13.62 \\
2.56 & 8.745 & 13.8 & 18.93 & 11.71 \\
3.06 & 8.325 & 14.19 & 20.5 & 10.14 \\
4.57 & 7.455 & 15.24 & 24.87 & 5.96 \\
5.82 & 6.78 & 17.25 & 27.45 & 3.54 \\
7.09 & 5.04 & 19.44 & 28.87 & 1.89 \\
8.34 & 4.17 & 21.48 & 30.05 & 0.89 \\
9.59 & 3.165 & 23.46 & 30.46 & 0.1 \\
10.86 & 2.415 & 25.8 & 30.99 & 0 \\
12.12 & 1.44 & 27.45 & 31.38 & 0 \\
13.39 & 0.765 & 28.47 & 31.3 & 0 \\
14.64 & 0.345 & 29.46 & 31.19 & 0 \\
15.91 & 0.06 & 29.94 & 31.35 & 0 \\
\hline
\end{tabular}

Table S24. Concentration vs time data for $30 \mathrm{mM} \mathrm{Ac3}$ with 1.00 equiv EDC. 


\begin{tabular}{rrrrr}
\hline Time $(\mathrm{min})$ & {$[\mathrm{An} 3](\mathrm{mM})$} & {$[\mathrm{Ac} 3](\mathrm{mM})$} & {$[\mathrm{EDU}](\mathrm{mM})$} & {$[\mathrm{EDC}](\mathrm{mM})$} \\
\hline 0.00 & 0 & 33.12 & 0 & \\
1.11 & 6.18 & 18.99 & 10.23 & 13.6 \\
1.61 & 6.945 & 16.71 & 12.19 & 10.94 \\
2.11 & 7.41 & 15.96 & 14.25 & 9.18 \\
2.61 & 7.755 & 16.02 & 16.17 & 7.77 \\
3.11 & 7.365 & 16.23 & 17.28 & 6.4 \\
4.62 & 6.255 & 18.15 & 20.31 & 3.41 \\
5.87 & 5.4 & 19.8 & 21.85 & 1.77 \\
7.12 & 4.35 & 22.08 & 23.06 & 0.83 \\
8.37 & 3.405 & 23.91 & 23.53 & 0.21 \\
9.64 & 2.445 & 26.19 & 24.18 & 0 \\
10.89 & 1.425 & 27.69 & 24.05 & 0 \\
12.14 & 0.6 & 29.04 & 23.86 & 0 \\
13.39 & 0.03 & 29.55 & 23.93 & 0 \\
14.49 & 0.06 & 29.91 & 24.05 & 0 \\
15.92 & 0.135 & 30.48 & 24.02 & 0 \\
\hline
\end{tabular}

Table S25. Concentration vs time data for $30 \mathrm{mM}$ Ac3 with 0.75 equiv EDC.

\begin{tabular}{rrrrr}
\hline Time $(\mathrm{min})$ & {$[\mathrm{An} 3](\mathrm{mM})$} & {$[\mathrm{Ac} 3](\mathrm{mM})$} & {$[\mathrm{EDU}](\mathrm{mM})$} & {$[\mathrm{EDC}](\mathrm{mM})$} \\
\hline 0.00 & 0 & 33.12 & 0 & \\
1.12 & 4.995 & 21.81 & 7.99 & 7.75 \\
1.64 & 5.4 & 20.19 & 9.7 & 5.88 \\
2.14 & 5.13 & 19.59 & 10.72 & 4.46 \\
2.64 & 5.535 & 20.01 & 11.93 & 3.51 \\
3.14 & 5.28 & 20.19 & 12.84 & 2.64 \\
3.66 & 5.025 & 20.58 & 13.68 & 2.06 \\
4.16 & 4.8 & 21.57 & 14.15 & 1.48 \\
4.66 & 4.62 & 22.02 & 14.62 & 1.1 \\
5.16 & 4.32 & 22.71 & 15.16 & 0.83 \\
5.66 & 3.78 & 23.88 & 15.16 & 0.48 \\
7.16 & 2.79 & 26.1 & 15.87 & 0.08 \\
8.41 & 1.395 & 27.9 & 15.83 & 0 \\
9.66 & 1.065 & 29.13 & 16.1 & 0 \\
10.92 & 0.825 & 30.12 & 16.03 & 0 \\
12.17 & 0.195 & 30.36 & 16.07 & 0 \\
13.44 & 0 & 30.81 & 15.84 & 0 \\
14.71 & 0 & 31.05 & 16.05 & 0 \\
15.97 & 0 & 31.26 & 16.01 & 0 \\
\hline
\end{tabular}

Table S26. Concentration vs time data for $30 \mathrm{mM} \mathrm{Ac3}$ with 0.50 equiv EDC. 


\begin{tabular}{rrrrr}
\hline Time $(\mathrm{min})$ & {$[\mathrm{An} 4](\mathrm{mM})$} & {$[\mathrm{Ac} 4](\mathrm{mM})$} & {$[\mathrm{EDU}](\mathrm{mM})$} & {$[\mathrm{EDC}](\mathrm{mM})$} \\
\hline 0 & 0 & 49.185 & 0 & \\
1.04 & 5.61375 & 37.635 & 11.37 & 8.5 \\
1.3 & 5.50125 & 37.455 & 12.96 & 7.18 \\
1.55 & 5.4 & 37.965 & 14.17 & 5.78 \\
1.8 & 5.20875 & 38.295 & 15.16 & 4.78 \\
2.05 & 4.875 & 38.61 & 16.23 & 3.87 \\
2.3 & 4.54125 & 40.095 & 16.94 & 3.15 \\
2.55 & 4.185 & 40.68 & 17.89 & 2.87 \\
2.8 & 4.125 & 40.965 & 18.25 & 1.93 \\
3.05 & 3.41625 & 42.6 & 18.75 & 1.38 \\
3.3 & 2.76375 & 43.305 & 19.2 & 1.14 \\
3.82 & 2.08125 & 44.67 & 19.54 & 0.47 \\
4.32 & 1.60125 & 45.885 & 19.97 & 0.06 \\
4.82 & 1.32 & 46.83 & 20.31 & 0 \\
5.32 & 0.6675 & 47.4 & 20.22 & 0 \\
5.84 & 0.70125 & 47.835 & 20.42 & 0 \\
6.84 & 0.345 & 48.165 & 20.46 & 0 \\
8.35 & 0.44625 & 48.6 & 20.77 & 0 \\
\hline
\end{tabular}

Table S27. Concentration vs time data for $45 \mathrm{mM}$ Ac4 with 0.50 equiv EDC.

\begin{tabular}{rrrrr}
\hline Time $(\mathrm{min})$ & {$[\mathrm{An} 4](\mathrm{mM})$} & {$[\mathrm{Ac4}](\mathrm{mM})$} & {$[\mathrm{EDU}](\mathrm{mM})$} & {$[\mathrm{EDC}](\mathrm{mM})$} \\
\hline 0 & 0 & 49.185 & 0 & \\
0.99 & 7.60125 & 34.14 & 15.01 & 16.73 \\
1.5 & 7.425 & 33.93 & 18.55 & 12.57 \\
2 & 6.9975 & 34.56 & 22.03 & 9.38 \\
2.5 & 6.17625 & 35.73 & 24.63 & 6.79 \\
3 & 5.39625 & 37.725 & 26.63 & 4.65 \\
3.5 & 5.33625 & 38.88 & 28.45 & 3 \\
4 & 4.3575 & 40.995 & 29.7 & 1.93 \\
4.52 & 3.0375 & 42.765 & 30.3 & 1.07 \\
5.02 & 2.35875 & 44.445 & 31.05 & 0.57 \\
5.54 & 1.5525 & 45.615 & 31.37 & 0.21 \\
6.02 & 1.1325 & 46.395 & 31.55 & 0.04 \\
6.52 & 1.1025 & 46.98 & 31.72 & 0.01 \\
7.02 & 0.7725 & 47.325 & 31.76 & 0 \\
7.52 & 0.345 & 47.97 & 31.62 & 0 \\
8.02 & 0.5325 & 48.09 & 31.78 & 0 \\
\hline
\end{tabular}

Table S28. Concentration vs time data for $45 \mathrm{mM}$ Ac4 with 0.75 equiv EDC. 


\begin{tabular}{rrrrr}
\hline Time $(\mathrm{min})$ & {$[\mathrm{An} 4](\mathrm{mM})$} & {$[\mathrm{Ac} 4](\mathrm{mM})$} & {$[\mathrm{EDU}](\mathrm{mM})$} & {$[\mathrm{EDC}](\mathrm{mM})$} \\
\hline 0 & 0 & 49.185 & 0 & \\
0.97 & 9.12375 & 30.525 & 18.58 & 25.87 \\
1.47 & 9.18 & 29.46 & 23.72 & 20.53 \\
1.97 & 8.71875 & 30.69 & 28.41 & 16.32 \\
2.47 & 7.88625 & 32.115 & 32.22 & 12.41 \\
2.99 & 6.9825 & 33.675 & 35.49 & 9.1 \\
3.5 & 6.225 & 35.085 & 38.38 & 6.6 \\
4 & 5.2275 & 36.885 & 40.65 & 4.48 \\
4.5 & 5.16375 & 39.06 & 42.31 & 2.97 \\
5 & 4.14375 & 40.71 & 43.29 & 1.82 \\
5.5 & 3.1425 & 42.6 & 44.29 & 1.03 \\
6 & 2.48625 & 44.07 & 44.69 & 0.59 \\
6.52 & 1.87125 & 45.3 & 45.05 & 0.27 \\
7.02 & 1.305 & 46.095 & 45.13 & 0.04 \\
7.52 & 1.45125 & 46.35 & 45.21 & 0.06 \\
8.02 & 0.945 & 46.995 & 45.06 & 0.01 \\
8.54 & 0.9225 & 47.55 & 45.34 & 0 \\
9.04 & 0.74625 & 47.49 & 45.16 & 0 \\
9.54 & 0.73875 & 47.505 & 45.38 & 0 \\
10.04 & 0.795 & 47.64 & 45.38 & 0 \\
10.54 & 1.0425 & 48.15 & 45.49 & 0 \\
11.05 & 0.95625 & 47.895 & 45.31 & 0 \\
11.55 & 0.73875 & 47.67 & 45.32 & 0 \\
12.05 & 0.5475 & 47.715 & 45.32 & 0 \\
\hline & & & &
\end{tabular}

Table S29. Concentration vs time data for $45 \mathrm{mM}$ Ac4 with 1.00 equiv EDC.

\begin{tabular}{rrrrr}
\hline Time $(\mathrm{min})$ & {$[\mathrm{An} 4](\mathrm{mM})$} & {$[\mathrm{Ac} 4](\mathrm{mM})$} & {$[\mathrm{EDU}](\mathrm{mM})$} & {$[\mathrm{EDC}](\mathrm{mM})$} \\
\hline 0.00 & 0 & 32.145 & 0 & \\
1.09 & 3.65625 & 24.435 & 12.01 & 18.99 \\
1.34 & 3.6525 & 24.24 & 13.86 & 16.87 \\
1.61 & 3.5175 & 24.375 & 15.8 & 15.03 \\
1.86 & 3.61125 & 24.525 & 17.29 & 13.29 \\
2.11 & 3.18375 & 25.185 & 18.97 & 11.76 \\
2.36 & 3.00375 & 25.62 & 20.26 & 10.29 \\
2.61 & 2.61375 & 26.025 & 21.71 & 9.05 \\
2.86 & 2.47875 & 26.4 & 22.93 & 7.8 \\
3.12 & 1.9725 & 26.925 & 23.74 & 6.69 \\
3.37 & 1.4625 & 27.555 & 24.77 & 5.69 \\
3.62 & 1.75125 & 27.735 & 25.87 & 4.91 \\
4.12 & 0.87 & 28.56 & 27.35 & 3.42 \\
4.62 & 0.78 & 29.19 & 28.66 & 2.46 \\
5.12 & 0.29625 & 29.55 & 29.25 & 1.61 \\
5.62 & 0.32625 & 29.925 & 30.1 & 1.13 \\
6.14 & 0.16125 & 30.06 & 30.3 & 0.73 \\
6.64 & 0.255 & 30.405 & 30.82 & 0.37 \\
7.14 & 0.1275 & 30.315 & 30.98 & 0.13 \\
\hline
\end{tabular}

Table S30. Concentration vs time data for $30 \mathrm{mM}$ Ac4 with 1.00 equiv EDC. 


\begin{tabular}{rrrrr}
\hline Time $(\mathrm{min})$ & {$[\mathrm{An} 4](\mathrm{mM})$} & {$[\mathrm{Ac} 4](\mathrm{mM})$} & {$[\mathrm{EDU}](\mathrm{mM})$} & {$[\mathrm{EDC}](\mathrm{mM})$} \\
\hline 0.00 & 0 & 32.145 & 0 & \\
0.97 & 2.895 & 25.92 & 8.84 & 14.66 \\
1.24 & 2.4225 & 25.965 & 10.13 & 12.74 \\
1.49 & 2.7 & 25.725 & 11.85 & 11.33 \\
1.74 & 2.7375 & 26.01 & 13.37 & 10.03 \\
1.99 & 2.4675 & 26.34 & 14.59 & 8.72 \\
2.24 & 2.0475 & 27.015 & 15.41 & 7.42 \\
2.49 & 1.7925 & 27.54 & 16.59 & 6.46 \\
2.74 & 1.65 & 27.93 & 17.46 & 5.52 \\
2.99 & 1.62 & 28.23 & 18.5 & 4.78 \\
3.24 & 1.185 & 28.725 & 19.3 & 4 \\
3.49 & 1.01625 & 29.205 & 19.95 & 3.31 \\
4.01 & 0.78 & 29.58 & 21.1 & 2.29 \\
4.51 & 0.49875 & 30 & 21.82 & 1.41 \\
5.01 & 0.4275 & 30.135 & 22.74 & 1.06 \\
6.02 & 0.1125 & 30.57 & 23.13 & 0.25 \\
6.54 & 0.0225 & 30.75 & 23.48 & 0.17 \\
\hline
\end{tabular}

Table S31. Concentration vs time data for $30 \mathrm{mM}$ Ac4 with 0.75 equiv EDC.

\begin{tabular}{rrrrr}
\hline Time $(\min )$ & {$[\mathrm{An} 4](\mathrm{mM})$} & {$[\mathrm{Ac} 4](\mathrm{mM})$} & {$[\mathrm{EDU}](\mathrm{mM})$} & {$[\mathrm{EDC}](\mathrm{mM})$} \\
\hline 0.00 & 0 & 32.145 & 0 & \\
0.99 & 1.72125 & 28.29 & 5.74 & 8.93 \\
1.24 & 1.83 & 27.87 & 7.01 & 7.85 \\
1.49 & 1.7325 & 27.96 & 7.83 & 6.69 \\
1.74 & 1.29375 & 28.29 & 8.54 & 5.6 \\
1.99 & 1.34625 & 28.725 & 9.68 & 4.95 \\
2.24 & 1.13625 & 29.04 & 10.36 & 4.15 \\
2.49 & 0.99375 & 29.205 & 10.96 & 3.45 \\
2.76 & 0.64125 & 29.79 & 11.36 & 2.79 \\
3.01 & 0.5325 & 30 & 11.83 & 2.32 \\
3.26 & 0.66375 & 29.865 & 12.67 & 2.08 \\
3.51 & 0.6525 & 30.18 & 12.98 & 1.67 \\
3.76 & 0.49125 & 30.51 & 13.32 & 1.34 \\
4.01 & 0.3975 & 30.585 & 13.73 & 1.09 \\
4.52 & 0.0825 & 31.2 & 14 & 0.57 \\
5.02 & 0.3 & 30.81 & 14.54 & 0.43 \\
5.52 & 0.06 & 31.335 & 14.55 & 0.17 \\
6.04 & 0.18375 & 31.23 & 14.81 & 0.03 \\
\hline
\end{tabular}

Table S32. Concentration vs time data for $30 \mathrm{mM}$ Ac4 with 0.50 equiv EDC. 


\begin{tabular}{rrrrr}
\hline Time (min) & {$[\mathrm{An} 4](\mathrm{mM})$} & {$[\mathrm{Ac} 4](\mathrm{mM})$} & {$[\mathrm{EDU}](\mathrm{mM})$} & {$[\mathrm{EDC}](\mathrm{mM})$} \\
\hline 0.00 & 0.00 & 77.12 & 0.00 & \\
1.27 & 11.58 & 51.27 & 21.96 & 14.77 \\
1.77 & 10.06 & 54.17 & 26.74 & 10.42 \\
2.27 & 10.22 & 53.82 & 31.06 & 7.35 \\
2.77 & 7.80 & 58.73 & 33.13 & 4.40 \\
3.27 & 5.46 & 62.36 & 34.95 & 2.52 \\
3.79 & 5.28 & 63.06 & 36.90 & 1.17 \\
4.29 & 4.75 & 65.27 & 37.54 & 0.20 \\
4.79 & 3.81 & 67.71 & 38.08 & 0.00 \\
5.29 & 2.94 & 69.02 & 38.47 & 0.00 \\
5.79 & 2.46 & 70.71 & 38.92 & 0.00 \\
6.29 & 1.78 & 71.46 & 38.45 & 0.00 \\
6.79 & 1.30 & 72.39 & 38.64 & 0.00 \\
7.31 & 1.24 & 72.59 & 38.60 & 0.00 \\
8.81 & 0.59 & 73.79 & 38.39 & 0.00 \\
9.81 & 0.51 & 73.79 & 38.65 & 0.00 \\
11.07 & 0.83 & 74.04 & 38.64 & 0.00 \\
12.32 & 0.585 & 73.995 & 38.56 & 0.00 \\
\hline
\end{tabular}

Table S33. Concentration vs time data for $75 \mathrm{mM}$ Ac4 with 0.50 equiv EDC. 


\section{References}

(1) Martin, R. B. Comparisons of indefinite self-association models. Chem. Rev. 1996, 96, 3043-3064.

(2) Chu, M.; Scioneaux, A. N.; Hartley, C. S. Solution-phase dimerization of an oblong shape-persistent macrocycle. f. Org. Chem. 2014, 79, 9009-9017.

(3) Kariyawasam, L. S.; Hartley, C. S. Dissipative assembly of aqueous carboxylic acid anhydrides fueled by carbodiimides. F. Am. Chem. Soc. 2017, 139, 11949-11955.

(4) Fox, J. In Applied Regression Analysis and Generalized Linear Models, 3rd Ed.; Sage Publishing: New York, 2016; Chapter 21, pp 587-606.

(5) Johnson, K. A.; Simpson, Z. B.; Blom, T. FitSpace Explorer: An algorithm to evaluate multidimensional parameter space in fitting kinetic data. Anal. Biochem. 2009, 387, 30-41. 